NBER WORKING PAPER SERIES

INFERENCE ON RISK PREMIA IN THE PRESENCE OF OMITTED FACTORS

\author{
Stefano Giglio \\ Dacheng Xiu \\ Working Paper 23527 \\ http://www.nber.org/papers/w23527 \\ NATIONAL BUREAU OF ECONOMIC RESEARCH \\ 1050 Massachusetts Avenue \\ Cambridge, MA 02138 \\ June 2017
}

We benefited tremendously from discussions with Jushan Bai, Svetlana Bryzgalova, John Cochrane, George Constantinides, Gene Fama, Valentin Haddad, Christian Hansen, Lars Hansen, Kris Jacobs, Raymond Kan, Serhiy Kozak, Nan Li, Toby Moskowitz, Stavros Panageas, Shri Santosh, Ivo Welch, Irina Zviadadze, and seminar and conference participants at the University of Chicago, Princeton University, Stanford University, Xiamen University, Tsinghua University, Hong Kong University of Science and Technology, Durham Business School, University of Liverpool Management School, University of Virginia-McIntire, University of Minnesota, Luxembourg School of Finance, University of British Columbia, Nankai University, Fudan University, the 2017 Annual meeting of the American Finance Association, the HEC-McGill Winter Finance Workshop, the 2017 Asian Meeting of the Econometric Society, the 2017 China Finance Review International Conference, and the BI-SHoF Conference. The views expressed herein are those of the authors and do not necessarily reflect the views of the National Bureau of Economic Research.

NBER working papers are circulated for discussion and comment purposes. They have not been peer-reviewed or been subject to the review by the NBER Board of Directors that accompanies official NBER publications.

(C) 2017 by Stefano Giglio and Dacheng Xiu. All rights reserved. Short sections of text, not to exceed two paragraphs, may be quoted without explicit permission provided that full credit, including $(\odot$ notice, is given to the source. 
Inference on Risk Premia in the Presence of Omitted Factors

Stefano Giglio and Dacheng Xiu

NBER Working Paper No. 23527

June 2017

JEL No. C32,C38,C55,C58,G12

\begin{abstract}
$\underline{\text { ABSTRACT }}$
We propose a three-pass method to estimate the risk premia of observable factors in a linear asset pricing model, which is valid even when the observed factors are just a subset of the true factors that drive asset prices or they are measured with error. We show that the risk premium of a factor can be identified in a linear factor model regardless of the rotation of the other control factors as long as they together span the space of true factors. Motivated by this rotation invariance result, our approach uses principal components to recover the factor space and combines the estimated principal components with each observed factor to obtain a consistent estimate of its risk premium. Our methodology also accounts for potential measurement error in the observed factors and detects when such factors are spurious or even useless. The methodology exploits the blessings of dimensionality, and we therefore apply it to a large panel of equity portfolios to estimate risk premia for several workhorse linear models. The estimates are robust to the choice of test portfolios within equities as well as across many asset classes.
\end{abstract}

\author{
Stefano Giglio \\ University of Chicago \\ Booth School of Business \\ 5807 S. Woodlawn Avenue \\ Chicago, IL 60637 \\ and NBER \\ stefano.giglio@chicagobooth.edu \\ Dacheng Xiu \\ Booth School of Business \\ University of Chicago \\ 5807 South Woodlaswn Avenue \\ Chicago, IL 60637 \\ dachxiu@ chicagobooth.edu
}




\title{
Inference on Risk Premia in the Presence of Omitted Factors*
}

\author{
Stefano Giglio ${ }^{\dagger}$ \\ Dacheng Xiu \\ Chicago Booth \\ Chicago Booth
}

First version: November 2016

This Version: June 2017

\begin{abstract}
We propose a three-pass method to estimate the risk premia of observable factors in a linear asset pricing model, which is valid even when the observed factors are just a subset of the true factors that drive asset prices or they are measured with error. We show that the risk premium of a factor can be identified in a linear factor model regardless of the rotation of the other control factors as long as they together span the space of true factors. Motivated by this rotation invariance result, our approach uses principal components to recover the factor space and combines the estimated principal components with each observed factor to obtain a consistent estimate of its risk premium. Our methodology also accounts for potential measurement error in the observed factors and detects when such factors are spurious or even useless. The methodology exploits the blessings of dimensionality, and we therefore apply it to a large panel of equity portfolios to estimate risk premia for several workhorse linear models. The estimates are robust to the choice of test portfolios within equities as well as across many asset classes.
\end{abstract}

Keywords: Three-Pass Estimator, Empirical Asset Pricing Models, PCA, Latent Factors, Omitted Factors, Measurement Error, Fama-MacBeth Regression

\section{Introduction}

Asset pricing models often predict that some factors - for example, intermediary capital or aggregate liquidity - should command a risk premium: investors should be compensated for their exposure to those sources of risk, holding constant their exposure to all other risk factors.

\footnotetext{
*We benefited tremendously from discussions with Jushan Bai, Svetlana Bryzgalova, John Cochrane, George Constantinides, Gene Fama, Valentin Haddad, Christian Hansen, Lars Hansen, Kris Jacobs, Raymond Kan, Serhiy Kozak, Nan Li, Toby Moskowitz, Stavros Panageas, Shri Santosh, Ivo Welch, Irina Zviadadze, and seminar and conference participants at the University of Chicago, Princeton University, Stanford University, Xiamen University, Tsinghua University, Hong Kong University of Science and Technology, Durham Business School, University of Liverpool Management School, University of Virginia-McIntire, University of Minnesota, Luxembourg School of Finance, University of British Columbia, Nankai University, Fudan University, the 2017 Annual meeting of the American Finance Association, the HEC-McGill Winter Finance Workshop, the 2017 Asian Meeting of the Econometric Society, the 2017 China Finance Review International Conference, and the BI-SHoF Conference.

${ }^{\dagger}$ Booth School of Business, University of Chicago. Address: 5807 S Woodlawn Avenue, Chicago IL 60637, USA. E-mail address: stefano.giglio@chicagobooth.edu.

${ }^{\ddagger}$ Booth School of Business, University of Chicago. Address: 5807 S Woodlawn Avenue, Chicago IL 60637, USA. E-mail address: dacheng.xiu@chicagobooth.edu.
} 
In many cases, the model-predicted factors are not tradable (i.e., they are not themselves traded portfolios). The risk premium for each factor can be estimated by constructing a portfolio with unit exposure (beta) to that factor and zero exposure to all other factors, for example via two-pass regressions like Fama-MacBeth or by constructing long-short portfolios with multiple sorts (isolating selected factor exposures nonparametrically); the risk premium is then computed as the average excess return of the portfolio exposed only to that factor, and not to the others. The risk premium of a non-tradable factor tells us how much investors are willing to pay to hedge that risk, and therefore represents quantitative evidence on the economic importance of that factor.

A fundamental concern when estimating risk premia via cross-sectional regressions is the potential omission of factors (noted, among others, by Jagannathan and Wang (1998)): for the estimation procedure to correctly recover the factor risk premia, all other priced factors in the economy need to be controlled for in the two-pass regression. This is an important problem in practice because most asset pricing models are too stylized to explicitly capture all sources of risk in the economy. ${ }^{1}$ The resulting omitted variable bias affects the estimated magnitude and even sign of the risk premia for the observed factors, and also the test for their statistical significance.

The typical, ad-hoc solution used in the literature to handle this omitted factor problem is to simply add some factors as "controls" (for example, the market return is often included even if when it is outside the theoretical model), or, alternatively, to add firm characteristics to the regressions. This solution involves selecting arbitrary factors or characteristics as controls, with no guidance from the model and no guarantee that the selected controls are the right ones; the results are often strongly dependent on the choice of additional factors to include.

Another important concern for risk premia estimation is measurement error. Theoretical asset pricing models often do not specify all the details of the construction of a factor, like aggregate liquidity or intermediary capital factor, so that measurement error is inevitable when working with those factors in empirical analysis. ${ }^{2}$ Similar to the omission of factors, the presence of measurement error in observable factors also results in a bias in standard estimation of risk premia.

In this paper, we show that by exploiting the large dimensionality of available test assets and a rotation invariance result for linear pricing models, we can correctly recover the risk premium of each observable factor, even when not all true risk factors are observed and included or perfectly measured. In other words, we can recover the slope of the ideal two-pass cross-sectional regression that includes errorfree observed factors as well as all the omitted factors - even when we cannot directly observe either. Our method therefore solves in a systematic way the omitted variable problem and the measurement error problem in estimating risk premia, avoiding the use of arbitrary choices for control factors or characteristics.

We first apply our methodology to a large set of 202 equity portfolios, sorted by different characteristics. We estimate and test the significance of the risk premia of tradable and non-tradable factors from

\footnotetext{
${ }^{1} \mathrm{~A}$ symptom of this omission is the fact that the pricing ability of the models is often poor, when tested using only the factors explicitly accounted for by the theory. This suggests that other factors may be present in the data that are not predicted by the model.

${ }^{2}$ As another example, in the setting of testing the CAPM, one of Roll's critiques (Roll (1977)) is that the market portfolio is not observable without error. The proxies constructed from equity portfolios, for example, deviate from the true market portfolio, and this implies that the mean-variance efficiency of the true market portfolio is untestable.
} 
a number of different models. We show that the conclusions about the magnitude and significance of the risk premia often depend on whether we account for omitted factors (using our estimator) or ignore them (using standard two-pass regressions). In contrast with the existing literature, we find a risk premium of the market portfolio that is positive, significant, and close to the time-series average of market excess returns, an indication of the validity of our procedure. We also decompose the variance of each observed factor into the components due to exposure to the underlying factors pricing the cross-section of returns, as well as the component due to measurement error. We find that several macroeconomic factors are dominated by noise, and after correcting for it and for exposures to unobservable factors, they command a risk premium of essentially zero. Instead, our results yield strong support for factors related to financial frictions (like the liquidity factor of Pástor and Stambaugh (2003)), whereas the standard methods that ignore omitted factors produce mixed or insignificant results for many of these factors in our sample.

We also show that our risk premia estimates remain similar when estimating them using 100 nonequity portfolios (options, bonds, currencies, commodities) in addition to or instead of equity portfolios. We show that once the unobservable factors that drive the different asset classes are accounted for, the risk premia for many factors are quite consistent with those estimated just using the cross-section of equities. This result suggests that indeed several common factors are priced in a consistent way across various asset classes, but this consistency is hard to detect without properly controlling for the unobservable factors to which various groups of assets are exposed.

Our solution to the omitted factor problem combines two-pass cross-sectional regressions with principal component analysis (PCA). The premise of our procedure is a simple but useful rotation invariance result that holds in linear factor models. Suppose that returns follow a linear factor model with $p$ factors and we wish to determine the risk premium of one of them (call it $g_{t}$ ). We show that a standard two-pass regression will always correctly recover the risk premium of $g_{t}$ as long as the two-pass analysis includes any $p-1$ "control" factors that, together with $g_{t}$, span the entire factor space. Because PCA recovers a factor-space rotation (as the number of assets $n \rightarrow \infty$ ), the factors extracted from PCA represent a natural set of "controls" that allow us to recover the risk premium of $g_{t}$. Using PCA also guarantees that the recovered "control" factors are measurement-error free (though subject to some estimation error), an important precondition for the controls to span the relevant space.

This invariance result is unique to estimating risk premia in linear asset pricing models, and it does not hold in standard regression settings with omitted variables. For example, in the standard regression setting where the researcher does not observe some variables but uses some linear combinations of the variables as "controls," the estimated coefficients for the observed variable are not invariant to rotations of the controls. ${ }^{3}$ The key difference is due to the fact that any rotation of the factors in

\footnotetext{
${ }^{3}$ A simple example may help clarify the intuition. Suppose that a variable $Y$ depends linearly on $X$ and $Z$, two correlated variables. We are interested in the coefficient on $X$. If $Z$ is not observed, the coefficient of a regression of $Y$ on $X$ alone will contain an omitted variable bias. Suppose $Z$ is not observed, but $X$ and a rotation of the two variables $(a X+b Z)$ are observed (where $a$ and $b$ are not known to the econometrician). Together, $X$ and $(a X+b Z)$ span the same space spanned by $X$ and $Z$. However, a regression of $Y$ on $X$ and $(a X+b Z)$ will not recover the correct coefficient on $X$, i.e., this regression does not solve the omitted variable bias. Our invariance result states that when $X$ and $Z$ are factors and $Y$ are returns in a linear factor model, the risk premium of $X$ is correctly identified even when the "control" factor is any linear combination $(a X+b Z)$.
} 
two-pass regressions has two offsetting effects on risk exposures and risk premia. The invariance result states that the two effects always offset each other so that risk premia for the observable factors are estimated correctly even when risk exposures are not, as long as the "controls" span the true factor space.

This invariance result is distinct from similar results the literature has explored in the past (e.g., Roll and Ross (1980), Huberman et al. (1987), Cochrane (2009)). This literature has explored the conditions under which rotations of a factor model retain the pricing ability of the original model. It has not, however, explored the properties of individual factors of the model. Our invariance result for the risk premium of an individual factor $g_{t}$ builds on the existing results to show that a particular invariance property holds not only for the pricing ability of the entire model, but for the risk premia of individual factors as well. This additional step is crucial when trying to understand the economic importance of a specific factor $g_{t}$ in the presence of omitted factors.

To apply directly, our invariance result requires error-free $g_{t}$ measurement, since $g_{t}$, together with the selected PCs, must span the entire factor space. In practice, it is likely that measurement error affects most empirical factors (and especially non-tradable ones). In this paper, we propose a three-pass estimator that exploits the invariance result while also accounting explicitly for potential measurement error in the observed factor. To do so, we first apply PCA to the set of returns, without using information in $g_{t}$; only then we relate the latent factors and their risk premia to the risk premium of the observable factor $g_{t}$. More specifically, we first use principal component analysis (PCA) to extract factors and their loadings from a large panel of testing portfolio returns; we then run a cross-sectional regression (CSR) to find the risk premia of the extracted factors, and finally recover the risk premia of the observable factor(s) from a time-series regression (TSR) that uncovers the relation between the observable and latent factors and eliminates the measurement error.

We show that our estimation procedure yields consistent risk-premium estimates for the observed factors, and we derive their asymptotic distribution when both the number of test portfolios $n$ and the number of observations $T$ are large. Our asymptotic theory allows for heteroscedasticity and correlation across both the time series and the cross-sectional dimensions, while explicitly accounting for the propagation of estimation errors through the multiple estimation steps. In addition, the increasing dimensionality simplifies the asymptotic variance of the risk-premium estimates, for which we also provide an estimator. We also construct a consistent estimator for the number of latent factors, while also showing that even without it, the risk-premium estimates could still be consistent. Finally, an advantage of our procedure is that inference remains valid even when any of the observable factors $g_{t}$ is spurious or even useless. In the paper, we also provide a test of the null that the observed factors $g_{t}$ are weak. Our methodology therefore provides a novel approach to inference in the presence of weak observable factors.

While most useful for estimating the economic importance of non-tradable factors, our methodology can also apply to tradable factors. For tradable factors, the risk premium can be computed in two ways. The first is to average the time-series excess return of the factor; the second is to use cross-sectional regressions, like two-pass estimators or our three-pass methodology, under the assumptions of the linear factor model. Misspecifications of the model (omitted controls; nonlinearities; correlated time-variation 
in risk exposures and risk premia) affect the latter estimator but not the former. Therefore, if the two estimates are different, it is an indication that the factor model is misspecified. While using standard two-pass regressions the two often differ (even for the market portfolio, see Lettau and Ludvigson (2001)), estimates of risk premia obtained with our three-pass methodology are close to the time series average returns for almost all of the tradable factors we study, including the market portfolio. This gives us confidence in using the same model assumptions to estimate the risk premia of non-tradable factors, for which this direct validation exercise is not feasible.

Finally, it is useful to point out that an alternative method to construct tradable portfolios from nontradable factors involves the projection of the factors onto the cross-section of testing portfolios, the so-called factor mimicking portfolio approach, see, e.g., Huberman et al. (1987). While the projection of the nontradable factors onto the return space does not change the pricing ability of the model, it rotates all the factors; as a consequence, the expected return of the projected factor does not correspond to the risk premium of the original nontradable factor, as clearly explained in Balduzzi and Robotti (2008). This is in contrast with the cross-sectional approaches like Fama-MacBeth that we employ in this paper, which construct a portfolio whose expected return is equal to the risk premium of the nontradable factor.

This paper sits at the confluence of several literatures, combining two-pass cross-sectional regressions with high-dimensional factor analysis.

Using two-pass regressions to estimate asset pricing models dates back to Black et al. (1972) and Fama and Macbeth (1973). Over the years, the econometric methodologies have been refined and extended; see for example Ferson and Harvey (1991), Shanken (1992), Jagannathan and Wang (1996), Welch (2008), and Lewellen et al. (2010). These papers, along with the majority of the literature, rely on large $T$ and fixed $n$ asymptotic analysis for statistical inference and only deal with models where all factors are specified and observable. Bai and Zhou (2015) and Gagliardini et al. (2016) extend the inferential theory to the large $n$ and large $T$ setting, which delivers better small-sample performance when $n$ is large relative to $T$. Connor et al. (2012) use semiparametric methods to model time variation in the risk exposures as function of observable characteristics, again when $n$ is large relative to $T$. Raponi et al. (2016) on the other hand study the ex-post risk premia using large $n$ and fixed $T$ asymptotics. For a review of this literature, see Shanken (1996), Jagannathan et al. (2010), and, more recently, Kan and Robotti (2012). Our asymptotic theory relies on a similar large $n$ and large $T$ analysis, yet we do not impose a fully specified model.

Our paper relates to the literature that has pointed out pitfalls in estimating and testing linear factor models. For instance, ignoring model misspecification and identification-failure leads to an overly positive assessment of the pricing performance of spurious (Kleibergen (2009)) or even useless factors (Kan and Zhang (1999a,b); Jagannathan and Wang (1998)), and biased risk premia estimates of true factors in the model. It is therefore more reliable to use inference methods that are robust to model misspecification (Shanken and Zhou (2007); Kan and Robotti (2008); Kleibergen (2009); Kan and Robotti (2009); Kan et al. (2013); Gospodinov et al. (2013); Kleibergen and Zhan (2014); Gospodinov et al. (2016); Bryzgalova (2015); Burnside (2016)). We study a different model misspecification form - priced factors omitted from the model, which would also bias the estimates for the observed factors. 
Hou and Kimmel (2006) argue that in this case, the definition of risk premia can be ambiguous. Relying on a large number of testing assets, our approach can provide consistent estimates of the risk premia without ambiguity, and detect spurious and useless factors. Lewellen et al. (2010) highlight the danger of focusing on a small cross section of assets with a strongly low-dimensional factor structure and suggest increasing the number of assets used to test the model. We point to an additional reason to use a large number of assets: to control properly for the missing factors in the cross-sectional regression. Moskowitz (2003) explores the relation between the characteristic-based portfolios and the covariance matrix of returns, which we parallel in our paper when we exploit the correlation of observable factors with the latent factors to estimate their risk premia; this link is also further developed in Pukthuanthong and Roll (2014), that propose, as part of their protocol for identifying valid factors, to use information about the canonical correlation of proposed factors and PCs of returns.

The literature on factor models has expanded dramatically since the seminal paper by Ross (1976) on arbitrage pricing theory (APT). Chamberlain and Rothschild (1983) extend this framework to approximate factor models. Connor and Korajczyk $(1986,1988)$ and Lehmann and Modest (1988) tackle estimation and testing in the APT setting by extracting principal components of returns, without having to specify the factors explicitly. More recently, Kozak et al. (2015) show how few principal components capture a large fraction of the cross-section of expected returns, which we will also show in our data. Clarke (2015) proposes an alternative way to construct statistical factors for returns, by sorting stocks into portfolios based on their conditonal expected return obtained from predictive regressions onto characteristics, and then by computing the principal components of these portfolios. Overall, one of the downsides of latent factor models is precisely the difficulty in interpreting the estimated risk premia. In our paper, we start from the same statistical intuition that we can use PCA to extract latent factors, but exploit it to estimate (interpretable) risk premia for the observable factors. Bai and Ng (2002) and Bai (2003) introduce asymptotic inferential theory on factor structures. In addition, Bai and $\mathrm{Ng}$ (2006b) propose a test for whether a set of observable factors spans the space of factors present in a large panel of returns. In contrast, our paper exploits statistically the spanning of the latent factors in time series, and their ability to explain the cross-sectional variation of expected returns.

Our paper also relates to the literature on weak factors (see for example Gospodinov et al. (2016) or Bryzgalova (2015)). An essential element of our rotation invariance result is that the space spanned by all priced factors can be recovered and factors spanning that space should be used as controls in the cross-sectional regression. Using principal component analysis to do so as we propose in this paper precludes the possibility of weak latent factors, i.e. latent factors that have positive risk premia but close to zero exposure in the set of test assets. Weak factors are associated with small eigenvalues of returns, and may not be selected by principal components. This is an important concern, that we do not tackle directly in our work and leave for future analysis. However, we point out three sources of robustness of our analysis with respect to the weak factors problem.

First, while our analysis requires that all true priced factors are pervasive, we allow for the factor of interest $g_{t}$ to be weak or even useless. Better yet, we derive a new Wald test for the null that the factor $g_{t}$ is weak. In our empirical work, we find that in fact several macro factors (like industrial production growth) are weak factors. Therefore, if $g_{t}$ is a weak factor, our procedure will classify it as such and 
make correct inference on its risk premium.

In addition, while our asymptotic variance results are correct only when the exact number of true factors is recovered, our estimates are still consistent even when "too many" principal components are extracted from the data. Therefore, if one is worried about the presence of weak factors in the data, increasing the number of principal components - adding factors with smaller and smaller eigenvalues will allow us to capture weaker and weaker factors and verify the robustness of the results.

Finally, we can look at tradable factors (for which a model-free estimate of the expected return can be computed directly) to verify that the risk premia estimated using our PCA-based methodology match the time-series average excess return of each factor; in the data, we find relatively small differences between the two approaches, suggesting that we are not omitting weak factors that might substantially bias our estimates.

Our analysis in this paper uses unconditional linear models. A large literature has explored conditional factor models where factor exposures, risk quantities, and risk premia can be time varying (for example, Jagannathan and Wang (1996) and Lewellen and Nagel (2006)). For the sake of clarity, we focus on unconditional models to illustrate the methodology. However, our analysis is still applicable to certain conditional models that allow for time-varying risk premia and risk exposures, by taking a stand on appropriate conditioning information, e.g., characteristics or state variables, at the cost of greater statistical complexity and potentially more severe curse of dimensionality.

Finally, our paper shares the same spirit with the vast literature on economic forecasting using factor models, as the first step towards forecasting typically involves a parsimonious representation of a large panel of predictors. PCA is a widely used toolkit for this purpose, see, e.g., Forni and Reichlin (1998), Stock and Watson (2002a), Stock and Watson (2002b), Bai and Ng (2006a), Bai and Ng (2008). Alternatively, Kelly and Pruitt (2013) and Kelly and Pruitt (2015) propose a three-pass regression filter which leads to a substantial improvement in forecasting, e.g., the conditional expectation of market returns.

Section 2 proposes a potentially misspecified beta-pricing model and sets the paper's objective. Section 3 presents an invariance result, which our identification strategy discussed in Section 4 relies on. Section 5 introduces the estimation procedure. Section 6 provides the asymptotic theory on inference. Section 7 presents Monte Carlo simulations, followed by an empirical study in Section 8. The appendix provides the mathematical proofs.

Throughout the paper, we use $(A: B)$ to denote the concatenation (by columns) of two matrices $A$ and $B . e_{i}$ is a vector with 1 in the $i$ th entry and 0 elsewhere, whose dimension depends on the context. $\iota_{k}$ denotes a $k$-dimensional vector with all entries being 1 . For any time series of vectors $\left\{a_{t}\right\}_{t=1}^{T}$, we denote $\bar{a}=\frac{1}{T} \sum_{t=1}^{T} a_{t}$. In addition, we write $\bar{a}_{t}=a_{t}-\bar{a}$. We use the capital letter $A$ to denote the matrix $\left(a_{1}: a_{2}: \ldots: a_{T}\right)$, and write $\bar{A}=A-\bar{a} \iota_{T}^{\top}$ correspondingly. We denote $\mathbb{P}_{A}=A\left(A^{\top} A\right)^{-1} A^{\top}$ and $\mathbb{M}_{A}=\mathbb{I}-\mathbb{P}_{A}$. 


\section{Model Setup}

We start describing a simple example - a special case of the more general setup considered later in this section - that illustrates the omitted factor and measurement error biases in two-pass regressions.

Suppose that we want to estimate and test the significance of the risk premium for an observable factor $g_{t}$ suggested by theory: for example, a liquidity or a financial intermediary capital factor. The true factor model includes $g_{t}$ but also another, unobserved, factor $f_{t}$. $g_{t}$ and $f_{t}$ can be arbitrarily correlated, and the betas with respect to each factor can also be arbitrarily cross-sectionally correlated, as long as they are not perfectly correlated. In addition, we allow for some measurement error in $g_{t}$.

Trying to estimate the risk premium of $g_{t}$ using standard cross-sectional regression methods (but without observing the potentially correlated $f_{t}$ ) causes two problems to arise. First, the time series regression of returns on the observed $g_{t}$ will yield biased betas (due both to the omission of $f_{t}$ and measurement error in $g_{t}$ ). The second pass involves a cross-sectional regression of the expected returns onto the estimated betas. Because only the betas corresponding to $g_{t}$ are included in the regression, another omitted variable bias arises. Eventually, all three biases appear in the estimated risk premium due to the time-series correlation of the factors, the cross-sectional correlation of the betas, and the measurement error in the observable factor $g_{t}$. In fact, it is enough that any of these issues occurs to bias the risk premium estimate of the factor of interest $g_{t}$.

Instead, our procedure is able to fully recover the correct risk premium of the error-free part of $g_{t}$, correcting all three sources of bias. To do so, it uses PCA on the panel of returns to extract factors that span the entire factor space (two factors in this case) and directly account for the variation in $g_{t}$ that is

not due to measurement error. Effectively, this methodology allows us to "control" for the unobservable factors in the risk premia estimation, and clean up the observed factors from the measurement error.

We specify that assets are priced by a linear factor model with potentially unobservable factors:

Assumption 1. Suppose that $f_{t}$ is a $p \times 1$ vector of asset pricing factors, and that $r_{t}$ denotes an $n \times 1$ vector of observable returns of the testing assets. The pricing model satisfies:

$$
r_{t}=\iota_{n} \gamma_{0}+\alpha+\beta \gamma+\beta v_{t}+u_{t}, \quad f_{t}=\mu+v_{t}, \quad \mathrm{E}\left(v_{t}\right)=\mathrm{E}\left(u_{t}\right)=0, \quad \text { and } \quad \operatorname{Cov}\left(u_{t}, v_{t}\right)=0,
$$

where $v_{t}$ is a $p \times 1$ vector of innovations of $f_{t}, u_{t}$ is a $n \times 1$ vector of idiosyncratic components, $\alpha$ is an $n \times 1$ vector of pricing errors, $\beta$ is an $n \times p$ factor loading matrix, and $\gamma_{0}$ and $\gamma$ are the zero-beta rate and the $p \times 1$ risk premia vector, respectively.

We allow for a non-zero pricing error $\alpha$ in the cross section of expected returns, so that the linear factor model is a potentially imperfect approximation of the true model. The focus of this paper is not on testing the null of APT, and allowing for at least some form of potential mispricings yields a more robust inference on the factor risk premia. We discuss in Section 6 which processes and types of pricing errors are allowed in our framework. Most of our results hold for non-stationary processes with heteroscedasticity and dependence in both the time series and the cross-sectional dimensions. 
Assumption 2. There is an observable $d \times 1$ vector, $g_{t}$, of factor proxies, which satisfies:

$$
g_{t}=\xi+\eta v_{t}+z_{t}, \quad \mathrm{E}\left(z_{t}\right)=0, \quad \text { and } \quad \operatorname{Cov}\left(z_{t}, v_{t}\right)=0,
$$

where $\eta$, the loading of $g$ on $v$, is a $d \times p$ matrix, $\xi$ is a $d \times 1$ constant, and $z_{t}$ is a $d \times 1$ measurement-error vector.

We allow for measurement error in $g_{t}$, because this is often plausible in practice. $z_{t}$ captures noise in the construction or measurement of the factors, or exposure to idiosyncratic risks (it can be correlated with $u_{t}$ ). Assumption 2 says that $g_{t}$ proxies for a set of asset pricing factors in the linear factor model representation: after removing measurement error, $g_{t}$ captures exactly a linear transformation of the fundamental factors, $\eta v_{t}$.

This specification implies that we can represent the true asset pricing model - after a rotation - as a model where $g_{t}$ corresponds to the first $d$ factors (after removing measurement error), together with other $p-d$ factors that are other combinations of the fundamental factors $v_{t}$ but are potentially not observed. The simple model discussed at the beginning of this section is a special case of the general model described in Assumptions 1 and 2.

\section{An Invariance Property}

We are interested in the risk premium associated with each observable factor in $g_{t}$. Recall that a factor's risk premium is the expected excess return of a portfolio with no idiosyncratic risk, no alpha, unit beta with respect to that factor, and zero beta with respect to all other risk factors. Because $g_{t}$ may contain measurement error, we refer to the risk premium of $g_{t}$ as the risk premium with respect to $\eta v_{t}$, (i.e., the compensation for the systematic risk to which $g_{t}$ is exposed). ${ }^{4}$ To calculate the risk premium of any of the factors in $g_{t}$, we rely on a rotation of the fundamental model (1) such that the factor appears directly as one of the $p$ factors, together with $p-1$ rotated factors. Such a rotation is not unique, but the risk premium is invariant to the rotation, as is shown below. This general result holds regardless of whether $v_{t}$ is observable or not.

Proposition 1. Suppose Assumptions 1 and 2 hold. The risk premium of $g_{t}$ is $\eta \gamma$. Moreover, it is invariant to the choice of factors in Assumption 1, as long as the space spanned by the rotated factors is the same as that of the true factors.

Proposition 1 states that we can always transform a linear factor model with $p$ factors $\left(v_{t}\right)$ into a representation where $g_{t}$ appears as one of the $p$ factors, together with $p-1$ other factors, that are linear combinations of the original factors. In any such transformation, as long as it preserves the same span of the factors, the risk premium of $g_{t}$ is equal to $\eta \gamma$. In contrast, the factor loading with respect to $g_{t}$ is obviously not invariant, and it depends on the correlation between $g_{t}$ and the other factors.

To see the intuition for the result, derived formally in the appendix, consider one observed factor $g_{t}$ with no constant or measurement error in it, so that $g_{t}=\eta v_{t}$. For any full-rank $p \times p$ matrix $H$, call

\footnotetext{
${ }^{4}$ Without ambiguity, we do not distinguish the risk premium of $\eta v_{t}$ from the risk premium of $g_{t}$.
} 
$q_{t}=H v_{t}$ the factors in the rotation $H$ of the linear factor model (i.e., in the factor model representation where $q_{t}$ are the factors). It is easy to observe that if the vector of risk premia of $v_{t}$ is $\gamma$, the vector of risk premia of $q_{t}$ is $H \gamma$. Now consider any rotations $H$ such that $g_{t}$ appears as a first factor. There are many such rotations: in fact, any matrix $H$ where the first row is $\eta$ will produce a rotated model where $g_{t}$ is the first factor (because $g_{t}=\eta v_{t}$ ). The risk premium of $g_{t}$ is then $\eta \gamma$, no matter what the other $p-1$ rows of $H$ are, because it is the first element of $H \gamma$. So the risk premium of $g_{t}(\eta \gamma)$ is well-defined in any rotation of the model where $g_{t}$ is the first factor, as long as any other $p-1$ linear combinations of $v_{t}$ are included (the additional rows of $H$ ). The risk exposures (betas) to $g_{t}$, instead, cannot be determined because they depend on the entire matrix $H$.

Proposition 1 also implies that in theory we can obtain the risk premium of $g_{t}$ in two ways, assuming $v_{t}$ is observed. We can first transform the model so that $g_{t}$ appears as a factor, and then apply standard two-pass estimator to this transformed model, directly recovering $\eta \gamma$ as the risk premium of $g_{t}$. Alternatively, we can first obtain the factor risk premia in the original model expressed in terms of $v_{t}$ (where $g_{t}$ may not directly appear), obtaining the risk premia of the factors $v_{t}, \gamma$. Then, we compute the risk premium of $g_{t}$ by multiplying this $\gamma$ by $\eta$, the exposure of $g_{t}$ to $v_{t}$.

Another implication of this invariance result is that as long as the original model (in terms of $v_{t}$ ) is well identified, then its rotations will also be well identified. For example, if $g_{t}$ and $h_{t}$ are two observable factors and are both linear functions of $v_{t}$, the risk premia associated with $g_{t}$ and $h_{t}$ will be well identified even if these two factors are highly correlated. Intuitively, rotating the model from $v_{t}$ to a model expressed in terms of $g_{t}$ and $h_{t}$ (and other factors) will rotate not only the factors and their risk premia but also the risk exposures. The rotations of factor exposures and factor risk premia offset each other, so that if the original model is well identified, the transformed model is also well identified. Our procedure exploits this invariance property to achieve identification of the risk premia of several observable factors even when they are highly correlated.

This invariance result effectively tells us that the risk premium of a factor $g_{t}$ can be identified as long as we control for the exposures to a set of factors that span the entire factor space, independently of their rotation. In this paper, we do not assume these factors are directly observable. In the next section, we discuss how to use PCA to identify the space spanned by these latent factors.

\section{Identification}

There is fundamental indeterminacy in latent factor models. We can multiply $\beta$ by any invertible matrix $H$ on the right-hand side, and multiply $\gamma$ and $v_{t}$ by $H^{-1}$ on the left-hand side, and both $\beta v_{t}$ and $\beta \gamma$ will remain the same. Clearly, this implies that it is not possible to directly identify $\gamma$ when not all factors are observed. The previous section shows that the risk premium associated with $g_{t}$ is always equal to $\eta \gamma$, no matter how the latent factors are rotated. So to estimate it, we need to recover a rotation of the factor space. Below we show that we can identify $\eta \gamma$ and recover it from observed variables, returns $r_{t}$ and the observable factor $g_{t}$, when $n \rightarrow \infty$.

Despite the potential unobserved heterogeneity due to $\alpha$, the demeaned time series of each asset return follows a standard approximate factor model (cf. Chamberlain and Rothschild (1983)), which, 
in matrix form, is given by

$$
\bar{R}=\beta \bar{V}+\bar{U}
$$

Bai and $\mathrm{Ng}$ (2002) discuss identifying the number of latent factors $p$ in a large $n$ and large $T$ setting. Bai (2003) argues that we can recover $\beta$ and $\bar{V}$ up to some invertible matrix $H$, only as $n \rightarrow \infty$. We denote them by $\beta H^{-1}$ and $H \bar{V}$. From the cross-sectional equation:

$$
\mathrm{E}\left(r_{t}\right)=\iota_{n} \gamma_{0}+\alpha+\beta \gamma=\iota_{n} \gamma_{0}+\alpha+\beta H^{-1} H \gamma
$$

we can recover $H \gamma$ and identify $\gamma_{0}$, if $\iota_{n}$ and $\beta$ are not perfectly correlated and the cross-sectional mean of $\alpha$ is zero. On the other hand, Assumption 2 leads to

$$
\bar{G}=\eta \bar{V}+\bar{Z}=\eta H^{-1} H \bar{V}+\bar{Z},
$$

so we can recover $\eta H^{-1}$ if $\bar{V} \bar{V}^{\top}$ is non-singular. This implies that we can identify $\eta \gamma=\eta H^{-1} H \gamma$.

The success of the identification strategy is another example of the "blessings of dimensionality" (Donoho (2000)). The large panel of cross-sectional returns certainly presents estimation challenges. However, it also provides a unique opportunity to identify and estimate the span of the latent factors that drive the asset returns. We can also identify and consistently estimate $\eta \gamma$, even without a consistent estimator of $p$, as long as we use some $\breve{p} \geq p$ in estimation in the same spirit of Moon and Weidner $(2015) .^{5}$

\section{The Three-Pass Estimator}

We summarize the parameters of interest in $\Gamma=\left(\gamma_{0}:(\eta \gamma)^{\top}\right)^{\top}$, where $\gamma_{0}$ is the zero-beta rate. We only use the observable data, (i.e., $r_{t}$ and $g_{t}, t=1,2, \ldots, T$ ). In light of the rotation invariance and identification results, we propose the following three-pass estimation procedure:

(i) PCA. Extract the principal components of returns, by conducting the PCA of the matrix $n^{-1} T^{-1} \bar{R}^{\top} \bar{R}$. Define the estimator for the factors and their loadings as:

$$
\widehat{V}=T^{1 / 2}\left(\xi_{1}: \xi_{2}: \ldots: \xi_{\widehat{p}}\right)^{\top}, \quad \text { and } \quad \widehat{\beta}=T^{-1} \bar{R} \widehat{V}^{\top}
$$

where $\xi_{1}, \xi_{2}, \ldots, \xi_{\widehat{p}}$ are the eigenvectors corresponding to the largest $\widehat{p}$ eigenvalues of the matrix $n^{-1} T^{-1} \bar{R}^{\top} \bar{R}$, and $\widehat{p}$ takes the following form:

$$
\widehat{p}=\arg \min _{1 \leq j \leq p_{\max }}\left(n^{-1} T^{-1} \lambda_{j}\left(\bar{R}^{\top} \bar{R}\right)+j \times \phi(n, T)\right)-1
$$

where $p_{\max }$ is some upper bound of $p$ and $\phi(n, T)$ is some penalty function.

\footnotetext{
${ }^{5}$ Bai (2009) discusses the identification of finite-dimensional parameters in a linear panel regression model with interactive fixed effects, also in the large $n$ and large $T$ setting with $p$ fixed. Allowing $p$ to increase with $n$ or $T$ is interesting, and we leave it for future work.
} 
(ii) CSR. Run a cross-sectional ordinary least square (OLS) regression of returns onto estimated factor loadings $\widehat{\beta}$ to obtain the risk premia of the estimated factors:

$$
\widetilde{\Gamma}:=\left(\widetilde{\gamma}_{0}, \widetilde{\gamma}^{\top}\right)^{\top}=\left(\left(\iota_{n}: \widehat{\beta}\right)^{\top}\left(\iota_{n}: \widehat{\beta}\right)\right)^{-1}\left(\iota_{n}: \widehat{\beta}\right)^{\top} \bar{r}
$$

(iii) TSR. Run another regression of $g_{t}$ onto the estimated factors based on (4), so that

$$
\widehat{\eta}=\bar{G} \widehat{V}^{\top}\left(\widehat{V} \widehat{V}^{\top}\right)^{-1}, \quad \text { and } \quad \widehat{G}=\widehat{\eta} \widehat{V}
$$

The estimator of the zero-beta rate and the risk premium for the observable factor $g_{t}$ is obtained by combining the estimates of the second and third steps, given by

$$
\widehat{\Gamma}:=\left(\begin{array}{c}
\widehat{\gamma}_{0} \\
\widehat{\gamma}
\end{array}\right):=\left(\begin{array}{cc}
1 & 0 \\
0 & \widehat{\eta}
\end{array}\right) \widetilde{\Gamma}=\left(\begin{array}{c}
\widetilde{\gamma}_{0} \\
\widehat{\eta} \widetilde{\gamma}
\end{array}\right) \text {. }
$$

This estimator also has a more compact form:

$$
\widehat{\gamma}_{0}=\left(\iota_{n}^{\top} \mathbb{M}_{\widehat{\beta}} \iota_{n}\right)^{-1} \iota_{n}^{\top} \mathbb{M}_{\widehat{\beta}} \bar{r}, \quad \text { and } \quad \widehat{\gamma}=\bar{G} \widehat{V}^{\top}\left(\widehat{V} \widehat{V}^{\top}\right)^{-1}\left(\widehat{\beta}^{\top} \mathbb{M}_{\iota_{n}} \widehat{\beta}\right)^{-1} \widehat{\beta}^{\top} \mathbb{M}_{\iota_{n}} \bar{r}
$$

The first step presents an estimator of $\widehat{p}$, which we will show to estimate $p$ consistently. This estimator is based on a penalty function, similar to the one Bai and $\mathrm{Ng}$ (2002) propose. It takes on a simpler form. $p_{\max }$ is an economically reasonable upper bound for the number of factors, imposed only to improve the finite sample performance. It is not needed in asymptotic theory. We prefer this estimator for its simplicity in proofs. Other estimators are equally applicable, including but not limited to those proposed by Onatski (2010) and Ahn and Horenstein (2013). Also, while Bai and Ng (2002) suggest PCA of the $n \times n$ matrix $n^{-1} T^{-1} \bar{R} \bar{R}^{\top}$ when $T>n$ to accelerate the algorithm, it is convenient to use the singular value decomposition of $n^{-1 / 2} T^{-1 / 2} \bar{R}$ instead, for which there exists efficient algorithms, regardless of the relative size of $n$ and $T$.

In the second stage, we suggest using an OLS regression for its simplicity. Either a generalized least squares (GLS) regression or a weighted least squares (WLS) regression is possible, but either of the two would require estimating a large number of parameters, (e.g., the covariance matrix of $u_{t}$ in GLS or its diagonal elements in WLS). As it turns out, these estimators will not necessarily improve the asymptotic efficiency of the OLS to the first order for the purpose of $\Gamma$ estimation. This is different from the standard large $T$ and fixed $n$ case because the covariance matrix of $u_{t}$ only matters at the order of $O_{p}\left(n^{-1}+T^{-1}\right)$, whereas the convergence rate of $\widehat{\Gamma}$ is $O_{p}\left(n^{-1 / 2}+T^{-1 / 2}\right) .{ }^{6}$

\footnotetext{
${ }^{6}$ It is also useful to note that since the principal components are portfolios of returns, using GLS would yield estimates of risk premia equivalent to the difference between the average returns of the PC portfolios and the estimated zero-beta rate. Under the additional assumption that the zero-beta rate is equal to the return of an observed portfolio like the T-bill rate, the second step could be substituted by an estimation of the average return of the PC portfolios. This would be equivalent to a cross-sectional GLS estimation of risk premia, with a constraint on the zero-beta rate. Estimates of the covariance matrix of returns, however, are still needed in case the zero-beta rate needs to be estimated when using GLS. In this paper, we choose to use OLS estimation in the second step because it allows to flexibly estimate both risk premia and the zero-beta rate without having to estimate the covariance matrix of returns, at no asymptotic efficiency cost.
} 
The third step is a new addition to the standard two-pass procedure. It is critical because it translates the uninterpretable risk premia of latent factors to those of factors the economic theory predicts. This step also removes the effect of measurement error, which the standard approach cannot accomplish. Even though $g_{t}$ can be multi-dimensional, the estimation for each observable factor is separate. Estimating the risk premium for one factor does not affect the estimation for the others at all, something that our estimator achieves without any omitted variable bias.

The three steps of our procedure suggest an alternative interpretation of the invariance result. As discussed in Cochrane (2009), in linear factor models the risk premium of any factor $g_{t}$ is simply the negative of the univariate covariance of $g_{t}$ with the stochastic discount factor $m_{t}$. Formally, in our setting, $m_{t}=\gamma_{0}^{-1}\left(1-\gamma^{\top} \Sigma_{v}^{-1} v_{t}\right)$, so that $-\gamma_{0} \operatorname{Cov}\left(g_{t}, m_{t}\right)=\eta \gamma$. The first two steps of the procedure (PCA and CSR) effectively recover the stochastic discount factor $m_{t}$ in the linear factor model (which is invariant to the rotation of the factors, as discussed in the existing literature, see, e.g., Roll and Ross (1980) and Huberman et al. (1987)). The requirement of spanning the factor space is what allows to estimate the stochastic discount factor consistently. The TSR step computes the univariate covariance between $g_{t}$ and the stochastic discount factor $m_{t}$ estimated in the first two stages. The invariance result follows from the fact that the latter stage only involves a univariate covariance with $m_{t}$, which itself is invariant to the rotation of the factor space.

\section{Asymptotic Theory}

In this section, we present the large sample distribution of our estimator as $n, T \rightarrow \infty$. Most results hold under the same or even weaker assumptions compared to those in Bai (2003). This is because our goals are different. Our main target is $\eta \gamma$, instead of the asymptotic distributions of factors and their loadings.

We need more notation. We use $\lambda_{j}(A), \lambda_{\min }(A)$, and $\lambda_{\max }(A)$ to denote the $j$ th, the minimum, and the maximum eigenvalues of a matrix $A$. By convention, $\lambda_{1}(A)=\lambda_{\max }(A)$. In addition, we use $\|A\|_{1},\|A\|_{\infty},\|A\|$, and $\|A\|_{\mathrm{F}}$ to denote the $\mathbb{L}_{1}$ norm, the $\mathbb{L}_{\infty}$ norm, the operator norm (or $\mathbb{L}_{2}$ norm), and the Frobenius norm of a matrix $A=\left(a_{i j}\right)$, that is, $\max _{j} \sum_{i}\left|a_{i j}\right|, \max _{i} \sum_{j}\left|a_{i j}\right|, \sqrt{\lambda_{\max }\left(A^{\top} A\right)}$, and $\sqrt{\operatorname{Tr}\left(A^{\top} A\right)}$, respectively. We also use $\|A\|_{\mathrm{MAX}}=\max _{i, j}\left|a_{i j}\right|$ to denote the $\mathbb{L}_{\infty}$ norm of $A$ on the vector space.

Let $(\mathrm{P}, \Omega, \mathcal{F})$ be the probability space. $K$ is a generic constant that may change from line to line. We say a sequence of centered multivariate random variables $\left\{y_{t}\right\}_{t \geq 1}$ satisfy the exponential-type tail condition, if there exist some constants $a$ and $b$, such that $\mathrm{P}\left(\left|y_{i t}\right|>y\right) \leq \exp \left\{-(y / b)^{a}\right\}$, for all $i$ and $t$. We say a sequence of random variables satisfy the strong mixing condition if the mixing coefficients satisfy $\alpha_{m} \leq \exp \left(-K m^{c}\right)$, for $m=1,2, \ldots$, and some constants $c>0$ and $K>0$. 


\subsection{Determining the Number of Factors}

We start with assumptions on the idiosyncratic component $u_{t}$. Define, for any $t, t^{\prime} \leq T, i, i^{\prime} \leq n$ :

$$
\gamma_{n, t t^{\prime}}=\mathrm{E}\left(n^{-1} \sum_{i=1}^{n} u_{i t} u_{i t^{\prime}}\right), \quad \mathrm{E}\left(u_{i t} u_{i^{\prime} t}\right)=\sigma_{i i^{\prime}, t}, \quad \text { and } \quad \mathrm{E}\left(u_{i t} u_{i^{\prime} t^{\prime}}\right)=\sigma_{i i^{\prime}, t t^{\prime}} .
$$

Assumption 3. There exists a positive constant $K$, such that for all $n$ and $T$,

$$
\begin{aligned}
& \text { (i) } T^{-1} \sum_{t=1}^{T} \sum_{t^{\prime}=1}^{T}\left|\gamma_{n, t t^{\prime}}\right| \leq K, \quad \gamma_{n, t t} \leq K . \\
& \text { (ii) }\left|\sigma_{i i^{\prime}, t}\right| \leq\left|\sigma_{i i^{\prime}}\right|, \quad \text { for some } \sigma_{i i^{\prime}} \text { and for all } t . \quad \text { In addition, } n^{-1} \sum_{i=1}^{n} \sum_{i^{\prime}=1}^{n}\left|\sigma_{i i^{\prime}}\right| \leq K . \\
& \text { (iii) } n^{-1} T^{-1} \sum_{i=1}^{n} \sum_{i^{\prime}=1}^{n} \sum_{t=1}^{T} \sum_{t^{\prime}=1}^{T}\left|\sigma_{i i^{\prime}, t t^{\prime}}\right| \leq K . \\
& \text { (iv) } \mathrm{E}\left(u_{t}^{\top} u_{t^{\prime}}-\mathrm{E} u_{t}^{\top} u_{t^{\prime}}\right)^{2} \leq K n, \quad \text { for all } t, t^{\prime} .
\end{aligned}
$$

Assumption 3 is similar to Assumption C in Bai (2003), which imposes restrictions on the temporal and cross-sectional dependence and heteroskedasticity of $u_{t}$. Stationarity of $u_{t}$ is not required. Eigenvalues of the residual covariance matrices $\mathrm{E}\left(u_{t} u_{t}^{\top}\right)$ are not necessarily bounded. In fact, they can grow at the rate $n^{1 / 2}$. Therefore, this assumption is weaker than those for an approximate factor model in Chamberlain and Rothschild (1983).

Assumption 4. The factor innovation $V$ satisfies:

$$
\|\bar{v}\|_{\mathrm{MAX}}=O_{p}\left(T^{-1 / 2}\right), \quad\left\|T^{-1} V V^{\top}-\Sigma^{v}\right\|_{\mathrm{MAX}}=O_{p}\left(T^{-1 / 2}\right),
$$

where $\Sigma^{v}$ is a $p \times p$ positive-definite matrix and $0<K_{1}<\lambda_{\min }\left(\Sigma^{v}\right) \leq \lambda_{\max }\left(\Sigma^{v}\right)<K_{2}<\infty$.

Assumption 4 imposes rather weak conditions on the time series behavior of the factors. It certainly holds if factors are stationary and satisfy the exponential-type tail condition and the strong mixing condition, see, Fan et al. (2013).

Assumption 5. The factor loadings matrix $\beta$ satisfies $\|\beta\|_{\mathrm{MAX}} \leq K$. Moreover,

$$
\left\|n^{-1} \beta^{\top} \beta-\Sigma^{\beta}\right\|=o(1), \quad \text { as } n \rightarrow \infty,
$$

where $\Sigma^{\beta}$ is a $p \times p$ positive-definite matrix and $0<K_{1}<\lambda_{\min }\left(\Sigma^{\beta}\right) \leq \lambda_{\max }\left(\Sigma^{\beta}\right)<K_{2}<\infty$.

Assumption 5 is the so-called pervasive condition for a factor model. It requires the factors to be sufficiently strong that most assets have non-negligible exposures. This is a key identification condition, which dictates that the eigenvalues corresponding to the factor components of the return covariance matrix grow rapidly at rate $n$, so that as $n$ increases they can be separated from the idiosyncratic component whose eigenvalues are bounded or grow at a lower rate. This assumption precludes weak 
but priced latent factors. Onatski (2012) develops the inference methodology in a framework that allows for weak factors using a Pitman-drift-like asymptotic device. We leave the case of weak latent factors for future work. However, we demonstrate the robustness of our empirical results with respect to the number of factors: the risk premia estimates and their significance remain similar even as more latent factors with lower eigenvalues are added to the estimation.

That said, our setup explicitly allows for weak observable factors. Whether $g_{t}$ is strong or weak can be captured by the signal-to-noise ratio of its relationship with the underlying factors $v_{t}$ (from equation (2)). If either $\eta=0$ ( $g_{t}$ is not a priced factor) or the factor is very noisy (measurement error $z_{t}$ dominates the $g_{t}$ variation) then $g_{t}$ will be weak, and returns exposures to $g_{t}$ will be small. Our procedure estimates equation (2) in the third pass and is therefore able to detect whether an observable proxy $g_{t}$ has zero or low exposures to the fundamental factors ( $\eta$ is small) or whether it is noisy $\left(z_{t}\right.$ is large), and corrects for it when estimating the risk premium. The $\mathrm{R}^{2}$ of that regression reveals how noisy $g$ is, which, as we report in our empirical analysis, varies substantially across factor proxies. In addition, we provide a Wald test for the null hypothesis that a factor $g$ is weak in Section 6.4. Our methodology provides an alternative solution to the weak-identification problem (Kleibergen (2009)), which can be applied when $n$ is large.

Our assumptions that the latent factors are pervasive, while observable factors can potentially be weak, are not in conflict with existing empirical evidence. It is known from the literature (e.g., Bernanke and Kuttner (2005) and Lucca and Moench (2015)) that the stock market and the bond market strongly react to Federal Reserve and Government policies and that macroeconomic risks affect equity premia; fundamental macroeconomic shocks seem to be pervasive. At the same time, we do not observe all fundamental economic shocks directly, and have instead to rely on observable proxies; these are well known to be weak in some cases, like for example industrial production (see for example Gospodinov et al. (2014) and Bryzgalova (2015)).

Finally, the loadings here are non-random for convenience. In contrast, Gagliardini et al. (2016) consider random loadings because of their sampling scheme from a continuum of assets. Our assumption is more commonly seen in the literature, see, Connor and Korajczyk (1988), Bai (2003), and Fan et al. (2013).

Theorem 1. Under Assumptions 1, 2, 3, 4, and 5, and suppose that as $n, T \rightarrow \infty, \phi(n, T) \rightarrow 0$, and $\phi(n, T) /\left(n^{-1 / 2}+T^{-1 / 2}\right) \rightarrow \infty$, we have $\widehat{p} \stackrel{p}{\longrightarrow} p$.

By a simple conditioning argument, we can assume that $\widehat{p}=p$ when developing the limiting distributions of the estimators, see Bai (2003). In the sequel, we assume $\widehat{p}=p$. Even though consistency cannot guarantee the recovery of the true number of factors in any finite sample, our derivation in Section 6.4 shows that as long as $p \leq \widehat{p} \leq K$ for some finite $K$, we can estimate the parameters $\Gamma$ consistently.

\subsection{Limiting Distribution of $\widehat{\Gamma}$}

In this section, we derive the asymptotic distribution of the estimator $\widehat{\Gamma}$. We need more assumptions that link the factor proxies $g_{t}$ to the latent factors $v_{t}$. 
Assumption 6. The residual innovation $Z$ satisfies:

$$
\|\bar{z}\|_{\mathrm{MAX}}=O_{p}\left(T^{-1 / 2}\right), \quad\left\|T^{-1} Z Z^{\top}-\Sigma^{z}\right\|_{\mathrm{MAX}}=O_{p}\left(T^{-1 / 2}\right),
$$

where $\Sigma^{z}$ is positive-definite and $0<K_{1}<\lambda_{\min }\left(\Sigma^{z}\right) \leq \lambda_{\max }\left(\Sigma^{z}\right)<K_{2}<\infty$. In addition,

$$
\left\|Z V^{\top}\right\|_{\mathrm{MAX}}=O_{p}\left(T^{1 / 2}\right)
$$

Similar to Assumption 4, Assumption 6 holds if $z_{t}$ is stationary, and satisfies the exponential-type tail condition and some strong mixing condition. It is more general than the i.i.d. assumption, so that it can be justified for non-tradable factor proxies in the empirical applications.

Assumption 7. For any $t \leq T$, and $i, j \leq p, l \leq d$, the following moment conditions hold:

$$
\begin{aligned}
& \text { (i) } \quad \mathrm{E}\left(\sum_{s=1}^{T} \sum_{k=1}^{n} v_{j s} u_{k s}\right)^{2} \leq K n T . \\
& \text { (ii) } \quad \mathrm{E} \sum_{k=1}^{n}\left(\sum_{s=1}^{T} v_{j s} u_{k s}\right)^{2} \leq K n T . \\
& \text { (iii) } \quad \mathrm{E}\left(\sum_{s=1}^{T} \sum_{k=1}^{n} v_{i s} u_{k s} \beta_{k j}\right)^{2} \leq K n T .
\end{aligned}
$$

Assumption 7 resembles Assumption D in Bai (2003). The variables in each summation have zero means, so that the required rate can be justified under more primitive assumptions. In fact, it holds trivially if $v_{t}$ and $u_{t}$ are independent.

Assumption 8. For any $t \leq T$, and $k \leq n, l \leq d$, define $\sigma_{l k, t}^{z u}=\mathrm{E}\left(z_{l t} u_{k t}\right)$. The following moment conditions hold:

$$
\begin{aligned}
& \text { (i) } \quad\left|\sigma_{l k, t}^{z u}\right| \leq\left|\sigma_{l k}^{z u}\right| \leq K, \quad \text { for some } \sigma_{l k}^{z u} \text { and for all t. In addition, } \sum_{k=1}^{n}\left|\sigma_{l k}^{z u}\right| \leq K \\
& \text { (ii) } \quad \mathrm{E} \sum_{k=1}^{n}\left(\sum_{s=1}^{T}\left(z_{l s} u_{k s}-\mathrm{E}\left(z_{l s} u_{k s}\right)\right)\right)^{2} \leq K n T . \\
& \text { (iii) } \quad \mathrm{E}\left(\sum_{s=1}^{T} \sum_{k=1}^{n}\left(z_{l s} u_{k s}-\mathrm{E}\left(z_{l s} u_{k s}\right)\right) \beta_{k j}\right)^{2} \leq K n T .
\end{aligned}
$$

Similar to Assumption 7, Assumption 8 specifies the restrictions on the covariances between the idiosyncratic components and the measurement error. If $z_{t}$ and $u_{t}$ are independent, (i) - (iii) are easy to verify. For a tradable portfolio factor in $g_{t}$, we can interpret its corresponding $z_{t}$ as certain undiversified idiosyncratic risk, since $z_{t}$ is a portfolio of $u_{t}$ as implied from Assumptions 1 and 2. It is thereby reasonable to allow for covariances between $z_{t}$ and $u_{t}$. For non-tradable factors, $z_{t} \mathrm{~s}$ can also be correlated with $u_{t}$ in general. 
Assumption 9. The cross-sectional pricing error $\alpha$ is i.i.d., independent of $u$ and $v$, with mean 0 , standard deviation $\sigma^{\alpha}>0$, and a finite fourth moment.

Assumption 9 dictates the behavior of pricing errors in model (1). There is a large body of literature on testing the APT by exploring the deviation of $\alpha$ from 0, including Connor and Korajczyk (1988), Gibbons et al. (1989), MacKinlay and Richardson (1991), and more recently, Pesaran and Yamagata (2012) and Fan et al. (2015). This is, however, not the focus of this paper. Empirically, the pricing errors may exist for many reasons such as limits to arbitrage, transaction costs, market inefficiency, and so on, so that we allow for a misspecified linear factor model. Gospodinov et al. (2014) and Kan et al. (2013) also consider this type of model misspecification in their two-pass cross-sectional regression setting.

Assumption 10. There exists a $p \times 1$ vector $\beta_{0}$, such that $\left\|n^{-1} \beta^{\top} \iota_{n}-\beta_{0}\right\|_{\mathrm{MAX}}=o(1)$. Moreover, the matrix

$$
\left(\begin{array}{cc}
1 & \beta_{0}^{\top} \\
\beta_{0} & \Sigma^{\beta}
\end{array}\right) \quad \text { is of full rank. }
$$

The convergence of $n^{-1} \beta^{\top} \iota_{n}$ in Assumption 10 resembles the law of large numbers for factor loadings. The rank condition ensures that in the limit the factor loadings are not perfectly correlated in the cross section, and in particular, that the zero-beta rate is identified.

Assumption 11. As $T \rightarrow \infty$, the following joint central limit theorem holds:

$$
T^{1 / 2}\left(\begin{array}{c}
T^{-1} \operatorname{vec}\left(Z V^{\top}\right) \\
\bar{v}
\end{array}\right) \stackrel{\mathcal{L}}{\longrightarrow} \mathcal{N}\left(\left(\begin{array}{l}
0 \\
0
\end{array}\right),\left(\begin{array}{ll}
\Pi_{11} & \Pi_{12} \\
\Pi_{12}^{\top} & \Pi_{22}
\end{array}\right)\right),
$$

where $\Pi_{11}, \Pi_{12}$, and $\Pi_{22}$ are $d p \times d p, d p \times p$, and $p \times p$ matrices, respectively, defined as:

$$
\begin{aligned}
& \Pi_{11}=\lim _{T \rightarrow \infty} \frac{1}{T} \mathrm{E}\left(\operatorname{vec}\left(Z V^{\top}\right) \operatorname{vec}\left(Z V^{\top}\right)^{\top}\right), \\
& \Pi_{12}=\lim _{T \rightarrow \infty} \frac{1}{T} \mathrm{E}\left(\operatorname{vec}\left(Z V^{\top}\right) \iota_{T}^{\top} V^{\top}\right), \\
& \Pi_{22}=\lim _{T \rightarrow \infty} \frac{1}{T} \mathrm{E}\left(V \iota_{T} \iota_{T}^{\top} V^{\top}\right) .
\end{aligned}
$$

Assumption 11 describes the joint asymptotic distribution of $Z V^{\top}$ and $V \iota_{T}$. Because the dimensions of these random processes are finite, this assumption is a fairly standard result of some central limit theorem for mixing processes, (e.g., Theorem 5.20 of White (2000)). Not surprisingly, it is stronger than Assumption 4, which is sufficient for identification and consistency.

We now present the main theorem of the paper:

Theorem 2. Under Assumptions 1 - 11, and suppose $\widehat{p} \stackrel{p}{\longrightarrow}$, then as $n, T \rightarrow \infty$, we have

$$
n^{1 / 2}\left(\widehat{\gamma}_{0}-\gamma_{0}\right) \stackrel{\mathcal{L}}{\longrightarrow} \mathcal{N}\left(0,\left(1-\beta_{0}^{\top}\left(\Sigma^{\beta}\right)^{-1} \beta_{0}\right)^{-1}\left(\sigma^{\alpha}\right)^{2}\right)
$$




$$
\left(T^{-1} \Phi+n^{-1} \Upsilon\right)^{-1 / 2}(\widehat{\gamma}-\eta \gamma) \stackrel{\mathcal{L}}{\longrightarrow} \mathcal{N}\left(0, \mathbb{I}_{d}\right)
$$

where the asymptotic covariance matrices $\Phi$ and $\Upsilon$ are given by

$$
\begin{aligned}
\Phi= & \left(\gamma^{\top}\left(\Sigma^{v}\right)^{-1} \otimes \mathbb{I}_{d}\right) \Pi_{11}\left(\left(\Sigma^{v}\right)^{-1} \gamma \otimes \mathbb{I}_{d}\right)+\left(\gamma^{\top}\left(\Sigma^{v}\right)^{-1} \otimes \mathbb{I}_{d}\right) \Pi_{12} \eta^{\top} \\
& +\eta \Pi_{21}\left(\left(\Sigma^{v}\right)^{-1} \gamma \otimes \mathbb{I}_{d}\right)+\eta \Pi_{22} \eta^{\top}, \quad \text { and } \\
\Upsilon= & \left(\sigma^{\alpha}\right)^{2} \eta\left(\Sigma^{\beta}-\beta_{0} \beta_{0}^{\top}\right)^{-1} \eta^{\top} .
\end{aligned}
$$

Remarkably, Theorem 2 does not impose any restrictions on the relative rates of $n$ and $T$. Moreover, the asymptotic covariance matrix does not depend on the covariance matrix of the residual $u_{t}$ or the estimation error of $\beta$. Their impact on the asymptotic variance is of higher orders. Therefore, for the inference on the risk premium of $g_{t}$, there is no need to estimate the large covariance matrix of $u_{t}$. This also implies that the usual GLS or WLS estimator would not necessarily improve the efficiency of the OLS estimator. The large cross section of testing assets extracts all the relevant factors from their time-series variations, which help correct the biases due to missing controls and measurement error.

\subsection{Goodness-of-Fit Measures}

To measure the goodness-of-fit in the cross-sectional of expected returns, we define the usual crosssectional $\mathrm{R}^{2}$ for the latent factors:

$$
\mathrm{R}_{v}^{2}=\frac{\gamma^{\top}\left(\Sigma^{\beta}-\beta_{0} \beta_{0}^{\top}\right) \gamma}{\left(\sigma^{\alpha}\right)^{2}+\gamma^{\top}\left(\Sigma^{\beta}-\beta_{0} \beta_{0}^{\top}\right) \gamma}
$$

To measure the signal-to-noise ratio of each observable factor, we define the time-series $\mathrm{R}^{2}$ for each observable factor $g(1 \times T)$, for the time-series regression of $g_{t}$ on the latent factors:

$$
\mathrm{R}_{g}^{2}=\frac{\eta \Sigma^{v} \eta^{\top}}{\eta \Sigma^{v} \eta^{\top}+\Sigma^{z}}, \quad \text { where } \eta \text { is a } 1 \times p \text { vector. }
$$

To calculate these measures in a sample, we use

$$
\widehat{\mathrm{R}}_{v}^{2}=\frac{\bar{r}^{\top} \mathbb{M}_{\iota_{n}} \widehat{\beta}\left(\widehat{\beta}^{\top} \mathbb{M}_{\iota_{n}} \widehat{\beta}\right)^{-1} \widehat{\beta}^{\top} \mathbb{M}_{\iota_{n}} \bar{r}}{\bar{r}^{\top} \mathbb{M}_{\iota_{n}} \bar{r}} \quad \text { and } \quad \widehat{\mathrm{R}}_{g}^{2}=\frac{\widehat{\eta} \widehat{V} \widehat{V}^{\top} \widehat{\eta}^{\top}}{\bar{G} \bar{G}^{\top}}, \quad \text { respectively, }
$$

where $\bar{G}=g-\bar{g}$ is a $1 \times T$ vector. We can consistently estimate the cross-sectional $\mathrm{R}^{2}$ for the latent factors as well as the time-series $\mathrm{R}^{2}$ for each observable factor.

Theorem 3. Under Assumptions 1 - 11, and suppose $\widehat{p} \stackrel{p}{\longrightarrow}$, then as $n, T \rightarrow \infty$, we have

$$
\widehat{\mathrm{R}}_{v}^{2} \stackrel{p}{\longrightarrow} \mathrm{R}_{v}^{2} \quad \text { and } \quad \widehat{\mathrm{R}}_{g}^{2} \stackrel{p}{\longrightarrow} \mathrm{R}_{g}^{2}
$$




\subsection{Robustness of the Choice of $p$}

Although $\widehat{p}$ is a consistent estimator of $p$, it is possible that in finite sample $\widehat{p} \neq p$. In fact, without a consistent estimator of $\widehat{p}$, as long as our choice, denoted by $\breve{p}$, is greater than or equal to $p$, the estimator based on $\breve{p}$, denoted by $\breve{\Gamma}=\left(\breve{\gamma}_{0}: \breve{\gamma}^{\top}\right)^{\top}$, is consistent. This result is similar in spirit to that of Moon and Weidner (2015), who establish that, for inference on the regression coefficients in a linear panel model with interactive fixed effect, it is not necessary to estimate $p$ consistently, as long as the number of factors we use, $\breve{p}$, is greater than or equal to $p$.

Theorem 4. Suppose Assumptions 1 - 11 hold. In addition, assume that $u_{t}$ is i.i.d. $\mathcal{N}\left(0,\left(\sigma^{u}\right)^{2} \mathbb{I}_{n}\right)$, independent of $z_{t}$ and $v_{t}$. If $\breve{p} \geq p$ and $\breve{p} \leq K$ as $n / T \rightarrow c \in(0, \infty)$, then $\breve{\Gamma}$ is a consistent estimator of $\left(\gamma_{0}:(\eta \gamma)^{\top}\right)^{\top}$, and it holds that

$$
\breve{\Gamma}-\widehat{\Gamma}=O_{p}\left(n^{-1 / 2}\right)
$$

The above theorem establishes the desired consistency of $\breve{\Gamma}$. While we cannot establish its asymptotic distribution, simulation exercises suggest that the differences between the asymptotic variances of $\breve{\Gamma}$ and $\widehat{\Gamma}$ are tiny. This is also the case for our empirical study.

To prove this result, we need much stronger assumptions on $u_{t}$. This is because the proof relies on the use of random matrix theory to analyze the eigenvalues and eigenvectors of large sample covariance matrices. The i.i.d. assumption is typically imposed in most scenarios. Even though there is a large literature on "universality" results, e.g., Tao (2012), which aim at relaxing the normality assumption, we maintain it so as to apply random matrix theory to variables that are shown to be uncorrelated.

\subsection{Limiting Distribution of $\widehat{g}_{t}$}

As discussed above, our framework allows for measurement error in the observable factor proxies $g$. Theorem 3 above proves that we can clean these errors up with identified latent factors. Moreover, we can conduct inference on $g$ at each $t$, given additional assumptions. Similar to Bai (2003), these assumptions are essential to derive the central limit result for the rotated factors and their loadings.

Assumption 12. The following conditions hold:

$$
\begin{aligned}
& \text { (i) } \sum_{t^{\prime}=1}^{T}\left|\gamma_{n, t t^{\prime}}\right| \leq K, \quad \text { for all } t . \\
& \text { (ii) } \sum_{i^{\prime}=1}^{n}\left|\sigma_{i i^{\prime}}\right| \leq K, \quad \text { for all } i .
\end{aligned}
$$

This assumption is identical to Assumption $\mathrm{E}$ in Bai (2003). It restricts the eigenvalues of $\mathrm{E}\left(u_{t} u_{t}^{\top}\right)$ and $\mathrm{E}\left(u_{t}^{\top} u_{t}\right)$ to be bounded as the dimension increases, because the $\mathbb{L}_{\infty}$-norm is stronger than the operator norm for symmetric matrices. 
Assumption 13. For each $t$, as $n \rightarrow \infty$,

$$
n^{-1 / 2} \beta^{\top} u_{t} \stackrel{\mathcal{L}}{\longrightarrow} \mathcal{N}\left(0, \Omega_{t}\right)
$$

where, writing $\beta=\left(\beta_{1}: \beta_{2}: \ldots: \beta_{n}\right)^{\top}$,

$$
\Omega_{t}=\lim _{n \rightarrow \infty} \frac{1}{n} \sum_{i=1}^{n} \sum_{i^{\prime}=1}^{n} \beta_{i} \beta_{i^{\prime}}^{\top} \mathrm{E}\left(u_{i t} u_{i^{\prime} t}\right) .
$$

Assumption 13 is identical to Assumption F3 in Bai (2003), which is used to describe the asymptotic distribution of $\widehat{v}_{t}$ at each point in time.

Theorem 5. Under Assumptions $1-8,11,12$, and 13, and suppose that $\widehat{p} \stackrel{p}{\longrightarrow} p$, then as $n, T \rightarrow \infty$, we have

$$
\Psi_{t}^{-1 / 2}\left(\widehat{g}_{t}-\eta v_{t}\right) \stackrel{\mathcal{L}}{\longrightarrow} \mathcal{N}\left(0, \mathbb{I}_{d}\right)
$$

where $\Psi_{t}=T^{-1} \Psi_{1 t}+n^{-1} \Psi_{2 t}$,

$$
\begin{aligned}
\Psi_{1 t}= & \left\{\left(v_{t}^{\top}\left(\Sigma^{v}\right)^{-1} \otimes \mathbb{I}_{d}\right) \Pi_{11}\left(\left(\Sigma^{v}\right)^{-1} v_{t} \otimes \mathbb{I}_{d}\right)-\left(v_{t}^{\top}\left(\Sigma^{v}\right)^{-1} \otimes \mathbb{I}_{d}\right) \Pi_{12} \eta^{\top}\right. \\
& \left.-\eta \Pi_{12}^{\top}\left(\left(\Sigma^{v}\right)^{-1} v_{t} \otimes \mathbb{I}_{d}\right)+\eta \Pi_{22} \eta^{\top}\right\}, \quad \text { and } \\
\Psi_{2 t}= & \eta\left(\Sigma^{\beta}\right)^{-1} \Omega_{t}\left(\Sigma^{\beta}\right)^{-1} \eta^{\top} .
\end{aligned}
$$

In Bai (2003), the latent factors can be estimated at the $n^{-1 / 2}$-rate, provided that $n^{1 / 2} T^{-1} \rightarrow 0$. In our setting, the estimation error consists of the errors in estimating $\widehat{\eta}$ and $\widehat{v}_{t}$. Because $\widehat{\eta}$ is estimated up to a $T^{-1 / 2}$-rate error which dominates $T^{-1}$ terms, the convergence rate of $\widehat{g}_{t}$ does not rely on any relationship between $n$ and $T$.

\subsection{Asymptotic Variances Estimation}

We develop consistent estimators of the asymptotic covariances in Theorems 2 and 5 . We can estimate them for inference on risk premia using:

$$
\begin{aligned}
& \widehat{\Phi}=\left(\widetilde{\gamma}^{\top}\left(\widehat{\Sigma}^{v}\right)^{-1} \otimes \mathbb{I}_{d}\right) \widehat{\Pi}_{11}\left(\left(\widehat{\Sigma}^{v}\right)^{-1} \widetilde{\gamma} \otimes \mathbb{I}_{d}\right)+\left(\widetilde{\gamma}^{\top}\left(\widehat{\Sigma}^{v}\right)^{-1} \otimes \mathbb{I}_{d}\right) \widehat{\Pi}_{12} \widehat{\eta}^{\top}+\widehat{\eta} \widehat{\Pi}_{21}\left(\left(\widehat{\Sigma}^{v}\right)^{-1} \widetilde{\gamma} \otimes \mathbb{I}_{d}\right)+\widehat{\eta} \widehat{\Pi}_{22} \widehat{\eta}^{\top}, \\
& \widehat{\Upsilon}={\widehat{\sigma^{\alpha}}}^{2} \widehat{\eta}\left(\widehat{\Sigma}^{\beta}-\widehat{\beta}_{0} \widehat{\beta}_{0}^{\top}\right)^{-1} \widehat{\eta}^{\top}
\end{aligned}
$$

where $\widehat{\Pi}_{11}, \widehat{\Pi}_{12}, \widehat{\Pi}_{22}$, are the HAC-type estimators of Newey and West (1987), defined as:

$$
\begin{aligned}
\widehat{\Pi}_{11}= & \frac{1}{T} \sum_{t=1}^{T} \operatorname{vec}\left(\widehat{z}_{t} \widehat{v}_{t}^{\top}\right) \operatorname{vec}\left(\widehat{z}_{t} \widehat{v}_{t}^{\top}\right)^{\top} \\
& +\frac{1}{T} \sum_{m=1}^{q} \sum_{t=m+1}^{T}\left(1-\frac{m}{q+1}\right)\left(\operatorname{vec}\left(\widehat{z}_{t-m} \widehat{v}_{t-m}^{\top}\right) \operatorname{vec}\left(\widehat{z}_{t} \widehat{v}_{t}^{\top}\right)^{\top}+\operatorname{vec}\left(\widehat{z}_{t} \widehat{v}_{t}^{\top}\right) \operatorname{vec}\left(\widehat{z}_{t-m} \widehat{v}_{t-m}^{\top}\right)^{\top}\right),
\end{aligned}
$$




$$
\begin{aligned}
& \widehat{\Pi}_{12}=\frac{1}{T} \sum_{t=1}^{T} \operatorname{vec}\left(\widehat{z}_{t} \widehat{v}_{t}^{\top}\right) \widehat{v}_{t}^{\top}+\frac{1}{T} \sum_{m=1}^{q} \sum_{t=m+1}^{T}\left(1-\frac{m}{q+1}\right)\left(\operatorname{vec}\left(\widehat{z}_{t-m} \widehat{v}_{t-m}^{\top}\right) \widehat{v}_{t}^{\top}+\operatorname{vec}\left(\widehat{z}_{t} \widehat{v}_{t}^{\top}\right) \widehat{v}_{t-m}^{\top}\right), \\
& \widehat{\Pi}_{22}=\frac{1}{T} \sum_{t=1}^{T} \widehat{v}_{t} \widehat{v}_{t}^{\top}+\frac{1}{T} \sum_{m=1}^{q} \sum_{t=m+1}^{T}\left(1-\frac{m}{q+1}\right)\left(\widehat{v}_{t-m} \widehat{v}_{t}^{\top}+\widehat{v}_{t} \widehat{v}_{t-m}^{\top}\right),
\end{aligned}
$$

and

$$
\widehat{Z}=\bar{G}-\widehat{\eta} \widehat{V}, \quad \widehat{\Sigma}^{\beta}=n^{-1} \widehat{\beta}^{\top} \widehat{\beta}, \quad \widehat{\Sigma}^{v}=T^{-1} \widehat{V}^{\top}, \quad \widehat{\beta}_{0}=n^{-1} \widehat{\beta}^{\top} \iota_{n}, \quad{\widehat{\sigma^{\alpha}}}^{2}=n^{-1}\left\|\bar{r}-\left(\iota_{n}: \widehat{\beta}\right) \widetilde{\Gamma}\right\|_{\mathrm{F}}^{2},
$$

with $q \rightarrow \infty, q\left(T^{-1 / 4}+n^{-1 / 4}\right) \rightarrow 0$, as $n, T \rightarrow \infty$.

To prove the validity of these estimators, we need additional assumptions, because the estimands are more complicated than the parameters of interest.

Assumption 14. The sequence of $\left\{u_{t}, v_{t}, z_{t}\right\}_{t \geq 1}$ is jointly strong mixing, and satisfies the exponentialtype tail condition. Moreover, for all $t^{\prime}, t \leq T$,

$$
\mathrm{E}\left(u_{t}^{\top} u_{t^{\prime}}-\mathrm{E} u_{t}^{\top} u_{t^{\prime}}\right)^{4} \leq K n^{2}, \quad \mathrm{E}\left\|\beta^{\top} u_{t}\right\|^{4} \leq K n^{2} .
$$

Assumption 14 ensures that the factors and their loadings are consistent up to some rotations under the max norm. Fan et al. (2011) and Fan et al. (2015) also adopt it.

Theorem 6. Under Assumptions 1 - 12, 14, and suppose that $\widehat{p} \stackrel{p}{\longrightarrow} p$, then as $n, T \rightarrow \infty, n^{-3} T \rightarrow 0$, $q\left(T^{-1 / 4}+n^{-1 / 4}\right) \rightarrow 0, \widehat{\Phi} \stackrel{p}{\longrightarrow} \Phi$ and $\widehat{\Upsilon} \stackrel{p}{\longrightarrow} \Upsilon$.

To estimate the asymptotic covariance matrices $\Psi_{1 t}$ and $\Psi_{2 t}$ in Theorem 5, we can simply replace $v_{t}, \Sigma^{v}, \Pi_{11}, \Pi_{12}, \Pi_{22}, \eta, \Sigma^{\beta}$ by their sample analogues, $\widehat{v}_{t}, \widehat{\Sigma}^{v}, \widehat{\Pi}_{11}, \widehat{\Pi}_{12}, \widehat{\Pi}_{22}, \widehat{\eta}, \widehat{\Sigma}^{\beta}$, in the $\widehat{\Psi}_{1 t}$ and $\widehat{\Psi}_{2 t}$ constructions. With respect to $\Omega_{t}$, we need to impose additional assumptions, because it is rather challenging to estimate, when we allow heteroskedasticity and correlation in both the time series and cross section.

We consider two scenarios that are relevant in practice.

Assumption 15. Either of the following assumptions holds:

(i) The innovation $u_{i t}$ is cross-sectionally independent, i.e., $\mathrm{E}\left(u_{i t} u_{j t}\right)=0$, for any $t \leq T, 1 \leq i \neq j \leq n$.

(ii) The innovation $u_{i t}$ is stationary, and its covariance matrix $\Sigma^{u}$ is sparse, i.e., there exists some $h \in[0,1 / 2)$, with $\omega_{T}=(\log n)^{1 / 2} T^{-1 / 2}+n^{-1 / 2}$, such that

$$
s_{n}=\max _{1 \leq i \leq n} \sum_{i^{\prime}=1}^{n}\left|\Sigma_{i i^{\prime}}^{u}\right|^{h}, \quad \text { where } \quad s_{n}=o_{p}\left(\left(\omega_{T}^{1-h}+n^{-1}+T^{-1}\right)^{-1}\right) .
$$

Under Assumption 15(i), (6) and its estimator can be rewritten as

$$
\Omega_{t}=\lim _{n \rightarrow \infty} \frac{1}{n} \sum_{i=1}^{n} \beta_{i} \beta_{i}^{\top} \mathrm{E}\left(u_{i t}^{2}\right), \quad \text { and } \quad \widehat{\Omega}_{t}=\frac{1}{n} \sum_{i=1}^{n} \widehat{\beta}_{i} \widehat{\beta}_{i}^{\top} \widehat{u}_{i t}^{2}
$$


where, writing $\widehat{U}=\left(\widehat{u}_{i t}\right), \widehat{U}:=\bar{R}-\widehat{\beta} \widehat{V}$.

With Assumption 15(ii), (6) and its estimator can be rewritten as

$$
\Omega=\lim _{n \rightarrow \infty} \frac{1}{n} \beta^{\top} \Sigma^{u} \beta, \quad \text { and } \quad \widehat{\Omega}_{t}=\widehat{\Omega}=\frac{1}{n} \widehat{\beta}^{\top} \widehat{\Sigma}^{u} \widehat{\beta},
$$

where, for $1 \leq i, i^{\prime} \leq n$,

$$
\widehat{\Sigma}_{i i^{\prime}}^{u}=\left\{\begin{array}{cc}
\widetilde{\Sigma}_{i i}^{u}, & i=i^{\prime} \\
s_{i i^{\prime}}\left(\Sigma_{i i^{\prime}}^{u}\right), & i \neq i^{\prime}
\end{array}, \quad \widetilde{\Sigma}^{u}=\frac{1}{T} \sum_{t=1}^{T} \widehat{u}_{t} \widehat{u}_{t}^{\top},\right.
$$

and $s_{i i^{\prime}}(z): \mathbb{R} \rightarrow \mathbb{R}$ is a general thresholding function with an entry dependent threshold $\tau_{i i^{\prime}}$ such that (i) $s_{i i^{\prime}}(z)=0$ if $|z|<\tau_{i i^{\prime}}$; (ii) $\left|s_{i i^{\prime}}(z)-z\right| \leq \tau_{i i^{\prime}}$; and (iii) $\left|s_{i i^{\prime}}(z)-z\right| \leq a \tau_{i i^{\prime}}^{2}$, if $|z|>b \tau_{i i^{\prime}}$, with some $a>0$ and $b>1 . \tau_{i i^{\prime}}$ can be chosen as:

$$
\tau_{i i^{\prime}}=c\left(\widehat{\Sigma}_{i i} \widehat{\Sigma}_{i^{\prime} i^{\prime}}\right)^{1 / 2} \omega_{T}, \quad \text { for some constant } c>0
$$

Bai and Liao (2013) adopt a similar estimator of $\Sigma^{u}$ for efficient estimation of factor models.

With estimators of their components constructed, our estimators for $\Psi_{1 t}$ and $\Psi_{2 t}$ are defined as:

$$
\begin{aligned}
\widehat{\Psi}_{1 t}= & T^{-1}\left\{\left(\widehat{v}_{t}^{\top}\left(\widehat{\Sigma}^{v}\right)^{-1} \otimes \mathbb{I}_{d}\right) \widehat{\Pi}_{11}\left(\left(\widehat{\Sigma}^{v}\right)^{-1} \widehat{v}_{t} \otimes \mathbb{I}_{d}\right)-\left(\widehat{v}_{t}^{\top}\left(\widehat{\Sigma}^{v}\right)^{-1} \otimes \mathbb{I}_{d}\right) \widehat{\Pi}_{12} \widehat{\eta}^{\top}-\widehat{\eta} \widehat{\Pi}_{12}^{\top}\left(\left(\widehat{\Sigma}^{v}\right)^{-1} \widehat{v}_{t} \otimes \mathbb{I}_{d}\right)\right. \\
& \left.+\widehat{\eta} \widehat{\Pi}_{22} \widehat{\eta}^{\top}\right\}, \\
\widehat{\Psi}_{2 t}= & n^{-1} \widehat{\eta}\left(\widehat{\Sigma}^{\beta}\right)^{-1} \widehat{\Omega}_{t}\left(\widehat{\Sigma}^{\beta}\right)^{-1} \widehat{\eta}^{\top},
\end{aligned}
$$

where $\widehat{\Omega}_{t}$ is given by either (7) or (8).

Theorem 7. Under Assumptions 1 - 15, we have

$$
\widehat{\Psi}_{1 t}-\Psi_{1 t} \stackrel{p}{\longrightarrow} 0, \quad \text { and } \quad \widehat{\Psi}_{2 t}-\Psi_{2 t} \stackrel{p}{\longrightarrow} 0
$$

\subsection{Testing the Strength of an Observed Factor}

If the measurement error component is too large, or the signal-to-noise ratio is too low, factors may not have enough comovement with test asset returns to produce a reliable estimate of the risk premium. Such factors are regarded as weak ones, as discussed in Kan and Zhang (1999a), Kleibergen (2009), Bryzgalova (2015), and Burnside (2016).

For any factor $g_{t}$, we can construct a test of the null that the factor is weak. Without loss of generality, it is sufficient to consider the $d=1$ case. To do so, we formulate the hypotheses $\mathbb{H}_{0}: \eta=0$ vs $\mathbb{H}_{1}: \eta \neq 0$, and construct a Wald Test. Our test statistic is given by

$$
\widehat{W}=T \widehat{\eta}\left(\widehat{\Sigma}_{v}^{-1} \widehat{\Pi}_{11} \widehat{\Sigma}_{v}^{-1}\right)^{-1} \widehat{\eta}^{\top}
$$

The next theorem establishes the desired size control and the consistency of the test. 
Theorem 8. Suppose $d=1$ and $\widehat{p} \stackrel{p}{\longrightarrow} p$. Under Assumptions 1-12, 14, and as $n, T \rightarrow \infty, n^{-2} T \rightarrow 0$, $q\left(T^{-1 / 4}+n^{-1 / 4}\right) \rightarrow 0$, we have

$$
\lim _{n, T \rightarrow \infty} \mathrm{P}\left(\widehat{W}>\chi_{\widehat{p}}^{2}\left(1-\alpha_{0}\right) \mid \mathbb{H}_{0}\right)=\alpha_{0}, \quad \text { and } \quad \lim _{n, T \rightarrow \infty} \mathrm{P}\left(\widehat{W}>\chi_{\widehat{p}}^{2}\left(1-\alpha_{0}\right) \mid \mathbb{H}_{1}\right)=1,
$$

where $\chi_{\widehat{p}}^{2}\left(1-\alpha_{0}\right)$ is the $\left(1-\alpha_{0}\right)$-quantile of the chi-squared distribution with $\widehat{p}$ degree of freedom.

\section{Simulations}

In this section, we study the finite sample performance of our inference procedure using Monte Carlo simulations. We consider a five-factor data-generating process, where the latent factors are calibrated to match the de-noised five Fama-French factors (RmRf, SMB, HML, RMW, CMA, see Fama and French (2015)) from our empirical study below. Suppose that we do not observe all five factors, but instead some noisy version of the three Fama-French factors (RmRf, SMB, HML, see Fama and French (1993)), plus a potentially spurious macro factor calibrated to industrial production growth (IP) in our empirical study. Our simulations, therefore, include both the issue of omitted factors and that of a spurious factor. We calibrate the parameters $\eta, \Sigma^{v}, \Sigma^{z}, \Sigma^{u},\left(\sigma^{\alpha}\right)^{2}, \beta_{0}$, and $\Sigma^{\beta}$ to exactly match their counterparts in the data (in our estimation of the Fama-French five-factor model). We then generate the realizations of $v_{t}, z_{t}, u_{t}, \alpha$, and $\beta$ from a multivariate normal using the calibrated means and covariances.

We report in Tables 1, 2, and 3 the bias and the root-mean-square error of the estimates using standard two-pass regressions and our three-pass approach. We choose different numbers of factors to estimate the model, $\breve{p}=4,5$, and 6 , whereas the true value is 5 . The five rows in each panel provide the results for the zero-beta rate, RmRf, SMB, HML, and IP, respectively. Throughout these tables, we find that the three-pass estimator with $\breve{p}=5$ outperforms the other estimators, in particular when $n$ and $T$ are large. Instead, the two-pass estimates have substantial biases. For example, the bias for the market factor premium is so large that its two-pass estimates are all negative (True + Bias $<0$ ) even when $n$ and $T$ are large, which actually matches what we find using real data and has been documented in the literature, as we discuss below. The three-pass estimator with $\breve{p}=4$ has an obvious bias, compared to the cases with $\breve{p}=5$ and 6 , because an omitted-factor problem still affects it ( 4 factors do not span the entire factor space).

We then plot in Figure 1 the histograms of the standardized risk premia estimates using FamaMacBeth standard errors for the two-pass estimator (left column) and the estimated asymptotic standard errors for the three-pass method with $\breve{p}=5$ (right column). ${ }^{7}$ The histograms on the left deviate substantially from the standard normal distribution, whereas those on the right match the normal distribution very well, which verifies our central limit results. There exist some small higher order biases for $\gamma_{0}$ and the market risk premium, which would disappear with a larger $n$ and $T$ in simulations not included here.

Next, we report in Table 4 the estimated number of factors. We choose $\phi(n, T)=K(\log n+$

\footnotetext{
${ }^{7}$ We have also implemented the standard errors of the two-pass estimators using the formula given by Bai and Zhou (2015), which provides desirable performance when both $n$ and $T$ are large. However, we do not find substantial differences compared to the Fama-MacBeth method, so we omit those histograms.
} 
$\log T)\left(n^{-1 / 2}+T^{-1 / 2}\right)$, where $K=0.5 \times \widehat{\lambda}, \widehat{\lambda}$ is the median of the first $p_{\max }$ eigenvalues of $n^{-1} T^{-1} \bar{R}^{\top} \bar{R}$. The median eigenvalue helps adjust the magnitude of the penalty function for better finite sample accuracy. Although the estimator is consistent, it cannot give the true number of factors without error, in particular when $n$ or $T$ is small, potentially due to the ad-hoc choice of tuning parameters. ${ }^{8}$ In the empirical study, we apply this estimator of $p$ and select slightly more factors to ensure the robustness of the estimates, as suggested by Theorem 4 .

Finally, we evaluate the size and power properties of the proposed test in Section 6.7. To check the size control, we create a purely noisy factor with $\eta=0$ and variance calibrated to be the average variance of the 4 factors we consider. The left panel of Figure 2 plots the histogram of the test statistic under the null against the density of a $\chi^{2}$-distribution with 5 degrees of freedom. To evaluate the power, we plot on the right panel of Figure 2 the average rejection probabilities against the signal-to-noise strength measured by $\mathrm{R}_{g}^{2}$ for a sequence of factors. These factors only load on the market factor, and share the same total variance calibrated to be the average variance as above, with different $\mathrm{R}_{g}^{2} \mathrm{~s}$ ranging from 0 to $10 \%$.

\section{Empirical Analysis}

In this section we apply our three-pass methodology to the cross-section of equities. We estimate the risk premia of several factors, both traded and not traded, and show how our results differ from standard two-pass cross-sectional regressions (or Fama-MacBeth regressions since we use their method for calculating standard errors), which ignore the potential omitted factors in the data.

\subsection{Data}

We conduct our empirical analysis on a large set of standard portfolios of U.S. equities, testing several asset pricing models that have focused on risk premia in equity markets. We target U.S. equities because of their better data quality and because they are available for a long time period. However, our methodology could be applied to any country or asset class.

We include in our analysis 202 portfolios: 25 portfolios sorted by size and book-to-market ratio, 17 industry portfolios, 25 portfolios sorted by operating profitability and investment, 25 portfolios sorted by size and variance, 35 portfolios sorted by size and net issuance, 25 portfolios sorted by size and accruals, 25 portfolios sorted by size and momentum, and 25 portfolios sorted by size and beta. This set of portfolios captures a vast cross section of anomalies and exposures to different factors; at the same time, they are easily available on Kenneth French's website, and therefore represent a natural starting point to illustrate our methodology. ${ }^{9}$

Although some of these portfolio returns have been available since 1926, we conduct most of our analysis on the period from July of 1963 to December of 2015 (630 months), for which all of the returns

\footnotetext{
${ }^{8}$ The eigenvalue ratio-based test by Ahn and Horenstein (2013) does not work well in our simulation setting because the first eigenvalue dominates the rest by a wide margin, so that their test often suggests 1 factor.

${ }^{9}$ See the description of all portfolio construction on Kenneth French's website: http://mba.tuck.dartmouth.edu/ pages/faculty/ken.french/data_library.html.
} 
are available. We perform the analysis at the monthly frequency, and work with factors that are available at the monthly frequency.

Although the asset-pricing literature has proposed an extremely large number of factors (McLean and Pontiff (2015); Harvey et al. (2016)), we focus here on a few representative ones. Recall that the observable factors $g_{t}$ in the three-pass methodology can be either an individual factor or groups of factors. We consider here both cases to illustrate the methodology; importantly, the risk premia estimates for any factors do not depend on whether other factors are included in $g_{t}$. Here is a list of models and corresponding observable factors $g_{t}$ included: ${ }^{10}$

1. Capital Asset Pricing Model $(C A P M)$ : the value-weighted market return, constructed from the Center for Research in Security Prices (CRSP) for all stocks listed on the NYSE, AMEX, or NASDAQ.

2. Fama-French three factors (FF3): in addition to the market return, the model includes SMB (size) and HML (value).

3. Carhart's four-factor model $(F F 4)$ that adds a momentum factor (MOM) to FF3.

4. Fama-French five-factor model (FF5), from Fama and French (2015). The model adds to FF3 RMW (operating profitability) and CMA (investment).

5. Four factors from the Q-factor model ( $H X Z$ ) of Hou et al. (2015), which include the market return, ME (size), IA (investment), ROE (profitability).

6. Betting-against-beta factor $(B A B)$ from Frazzini and Pedersen (2014).

7. Quality-minus-junk factor ( $Q M J)$ from Asness et al. (2013).

8. Industrial production growth $(I P)$. Industrial production is a macroeconomic factor available for the entire sample period at the monthly frequency. We use $\operatorname{AR}(1)$ innovations as the factor.

9. The first three principal components of 279 macro-finance variables constructed by Ludvigson and $\mathrm{Ng}(2009)(L N)$, also available at the monthly frequency. We estimate a $\operatorname{VAR}(1)$ with those three principal components, and use innovations as factors.

10. The liquidity factor from Pástor and Stambaugh (2003).

11. Two intermediary capital factors, one from He et al. (2016) and one from Adrian et al. (2014).

\subsection{Factors from the Large Panel of Returns}

The first step for estimating the observable factor risk premia is to determine the latent factor model dimension, $p$. Figure 3 (left panel) reports the first eight eigenvalues of the covariance matrix of returns

\footnotetext{
${ }^{10}$ Factor time series for models 1-4 are obtained from Kenneth French's website; for model 5, from Lu Zhang; for models 6-7, from AQR's website; for model 8, from the Federal Reserve Bank of St. Louis; for model 9, from Sydney Ludvigson's website; for model 10, from Lubos Pastor's website; for model 11, from Bryan Kelly's website.
} 
for our panel of 202 portfolios. As typical for large panels, the first eigenvalue tends to be much larger than the others, so on the right panel we plot the eigenvalues excluding the first one. We observe a noticeable decrease in the eigenvalues after four and six factors, and our estimator suggests using four factors. As discussed in Section 6, our analysis is consistent as long as the number of factors $\hat{p}$ is at least as large as the true dimension $p$; to show the robustness of our results, we report the estimates separately using four, five, and six factors. The analysis is robust to using more factors.

After extracting the factors via PCA, the second pass in the three-pass procedure estimates the risk premia of the latent factors via cross-sectional regressions (CSR). We cannot interpret these risk premia in economic terms, as opposed to the risk premia of observable factors, because the PCs themselves do not have economic interpretations. The estimated zero-beta rate from the APT model is 55bp per month, close to the 40bp of the average T-bill return over the sample.

The model has a cross-sectional $\mathrm{R}_{v}^{2}$ of $65 \%$, indicating that it accounts for much of the cross-sectional variation in expected returns for the 202 test portfolios, but leaving some unexplained variation. This number is comparable with $73 \%$ cross-sectional $\mathrm{R}^{2}$ one obtains using the FF3 model on the cross-section of 25 portfolios sorted by size and book-to-market, yet, we obtain it for a cross-section almost ten times as large. We report in Figure 4 the actual and predicted excess returns for the model. Each panel of the figure highlights one of the eight test-asset groups that comprise our total of 202 portfolios. The fit is better for some groups of assets (FF25 and momentum) than others (industry), but overall the factor model with six factors performs relatively well.

\subsection{Risk Premia Estimates for Observable Factors}

Tables 5 and 6 report the estimates of observable factor risk premia. Each factor (or set of factors) $g_{t}$ corresponds to a panel of the tables; the tested $g_{t}$ appears in the first column. In each panel, the rows correspond to the coefficients of the cross-sectional regressions (intercept $\hat{\gamma}_{0}$ and the risk premia corresponding to the factors $\left.g_{t}, \hat{\eta} \hat{\gamma}\right)$. The number of observations $T$ is $630 \mathrm{in}$ all cases except for the $H X Z$ model (where $T=588)$, the macroeconomic factors from $L N(T=580)$ and the intermediary-capital model $(T=516)$.

Across columns, the tables report information about the average returns of the factors (when traded), standard Fama-MacBeth estimates of the risk premia that ignore potential omitted factors, and results of the three-pass procedure using different numbers of latent factors, from four to six.

To illustrate the table content, consider for example the second panel, corresponding to the FamaFrench three-factor model. The first column reports the average monthly returns for the three factors (RmRf, SMB, HML) over the sample period: respectively 50bp, 23bp, and 34bp. The number in the "intercept" row reports the average value of the T-bill rate $R_{f}$ over the sample period (in this case, 40bp).

The second set of columns corresponds to the standard Fama-MacBeth estimation of the intercept and the three risk premia using all of the 202 portfolios. The results of this exercise line up well with the previous literature. The zero-beta rate estimate is approximately $1.5 \%$ per month, more than $100 \mathrm{bp}$ higher than the average risk-free rate. The risk premium estimate associated with the market return is

negative, and significantly so. HML has a high and significant risk premium of $23 \mathrm{bp}$ per month, close to 
the time-series average return of the HML portfolio. Finally, size (SMB) has a smaller and statistically insignificant risk premium.

The remainder of the tables report the estimates for the three-pass procedure. As discussed above, we repeat the exercise for $\breve{p}=4,5$, and 6 . The estimates are stable across the number of factors used, consistent with the theoretical result that adding extra factors does not affect the validity of our procedure.

Finally, the last column also reports the p-value for the test of the null that each factor $g_{t}$ is weak, as described in Section 6.7. ${ }^{11}$ A rejection of the null indicates that $g_{t}$ is a strong factor for the cross-section of test portfolios.

The estimates of the zero-beta rate and the risk premia for the three factors differ substantially from the estimates obtained using the standard Fama-MacBeth regression. Consider the results for $\breve{p}=4$ (results for other values of $\breve{p}$ are similar as the table shows). First, the zero-beta rate estimate is 55bp, just 15bp per month above the risk-free rate. Second, the market risk premium estimate is positive, significant, and of a magnitude close to the average return in the data (the risk premium estimate is $37 \mathrm{bp}$ in the model, whereas the average return of the market portfolio in the data is 50bp over the risk-free rate, and 35bp over the estimated zero-beta rate). The risk premium associated with HML is stable at a significant $21 \mathrm{bp}$; the risk premium associated with size is significant and equal to $23 \mathrm{bp}$, matching exactly the average return in the data. Results for the FF3 model, therefore, are substantially different when estimated via Fama-MacBeth regression or via three-pass regressions.

The third column of each of the three-pass results reports the $\mathrm{R}^{2}$ of the time-series regression (TSR) of the observed $g_{t}$ onto the latent factors; we refer to this as $\mathrm{R}_{g}^{2}$. Recall that if the factors driving the returns' cross section entirely span $g_{t}$, we should expect to find $\mathrm{R}_{g}^{2}$ close to $100 \%$. However, if the observed $g_{t}$ is just a noisy proxy for some of the fundamental factors, this $\mathrm{R}_{g}^{2}$ will reflect the amount of noise in the observed $g_{t}$. In the data, we find interesting heterogeneity among the three factors of FF3 with respect to their $\mathrm{R}_{g}^{2}$. The market and size portfolios have $\mathrm{R}_{g}^{2}$ close to $100 \%$; HML displays greater noise, with an estimated $\mathrm{R}_{g}^{2}$ of about $67 \%$.

Figure 5 shows the time series of cumulated innovations in the original and cleaned (i.e., without measurement error) factors for the Fama-French three-factor model. The figures present a graphical representation of the variation in the original factors captured by the principal components, corresponding to the $\mathrm{R}_{g}^{2}$ reported in the table. The figure shows that all three factors correlate highly with the estimated latent factors.

Tables 5 and 6 report the results for the remaining factors and factor models we study, both traded factors (e.g., MOM) and non-traded factors (e.g., IP). We summarize here the main results, highlighting in particular the differences that emerge when estimating the model using our three-pass procedure rather than the standard Fama-MacBeth regression that is potentially affected by omitted factor bias.

Zero-beta rate. Whereas for most of the models estimated via standard Fama-MacBeth two-pass regression the zero-beta rate is much larger than the observed risk-free rate (typically between 50 and

\footnotetext{
${ }^{11}$ We use $\breve{p}=6$ for this test, corresponding to the rightmost set of results in the table, but results are similar for all values of $\breve{p}$.
} 
100bp above it), the zero-beta rate estimated from the three-pass procedure is mostly 15-20bp greater than the risk-free rate on average, and statistically insignificantly so. This is due to the fact that the latent model (with four to six factors) is able to capture a greater fraction of the overall level of equity-portfolio risk premia.

The market risk premium. A classic result in the empirical asset pricing literature is the typically negative estimate of the risk premium for market risk from cross-sectional regressions. This result highlights a potential misspecification for these regressions: under the assumptions of a linear factor model, for tradable factors the cross-sectional estimate of the risk premium should correspond to the time-series estimate of the average excess return of the portfolio.

The three-pass approach allows us to control for more factors beyond the observable ones, and at the same time exploit the beta spread across the 202 portfolios to pin down the risk premium of each observable factor better. The result is that the risk premium estimate for exposure to the aggregate stock market is positive and significant at 37bp, close to the average excess return of the market portfolio. It is also useful to note that our procedure guarantees that the estimated risk premium for a factor does not depend on whether it is estimated together with other observable factors or by itself; therefore, the market risk premium will be the same when estimating the $C A P M$ or the Fama-French three-factor and five-factor models.

We can also investigate the relationship between market beta and expected returns after controlling for the explanatory power of the omitted factors using a residual regression approach. ${ }^{12}$ Figure 6 plots the expected return vs. market beta, after partialing out the component explained by the other factors. The left panel uses a standard Fama-MacBeth estimator within a Fama-French 3-factor model, whereas the right panel uses our three-pass methodology. ${ }^{13}$ The solid red line in each graph corresponds to the slope estimated from the historical average return of the market, whereas the dashed line corresponds to the fitted line, i.e., the cross-sectional estimate of the slope. As discussed above, if the model is correctly specified, the two lines should overlap.

The figure shows that indeed, once the omitted factors are accounted for, there is a clear positive relationship between market beta and expected returns, and the slope is close to the average excess return of the market portfolio. Overall, the fact that the market risk premium significantly changes sign depending on whether we control for omitted factors serves as a strong warning that omitting factors could have important effects on our statistical and economic conclusions about the pricing of aggregate risks.

\footnotetext{
${ }^{12}$ In particular, recall that the estimate of the market risk premium using cross-sectional methods is the slope of a regression of average returns onto the betas of returns with the market and the control factors. It is well known that the slope of such a regression with respect to a specific factor (in this case, the market) can be also obtained by first regressing the outcome variable (average returns) and regressor of interest (market beta) on the remaining regressors (the other betas), and in a second stage regressing the residuals of the two regressions against each other. In this way, we can first partial out the component of the cross-section of expected returns and of market betas explained by the control factors, and then study the univariate relation between the residuals.

${ }^{13}$ It is worth noting that both the partial expected return and the partial betas - and therefore this entire graph - are also invariant to the rotation of the control factors.
} 
Other tradable factors. The table shows that using the three-pass method, the cross-sectional risk premia estimates for tradable factors are close to the time-series average excess returns of the portfolios themselves - not only for the market portfolio as described above, but for the vast majority of the tradable factors we examine. For example, the risk premium associated with HML is close to zero in the FF5 model when estimating it using standard two-pass regression, while it is positive, significant, and close to the time-series average return when using the three-pass method.

This result is important because it helps rule out misspecifications of our linear factor model. For tradable factors, risk premia can be computed in two ways: by estimating the time-series average excess return of the factor (a model-free estimator), or by computing the slope of two- and three-pass estimators under the assumptions of the linear factor model. Any misspecification that affects our methodology would bias the latter but not the former. Comparing the two estimates when possible (i.e., for tradable factors) is therefore a simple way to assess whether different types of misspecification - for example, factors with low variance and high risk premia missed by the PCA analysis, nonlinearities, correlated time variation in betas and risk premia - affect our estimator, at least as far as the tradable factors are concerned. The fact that we do not see economically large differences between the two estimators for tradable factors mitigates the misspecification concerns for non-tradable factors (for which this form of validation is not possible).

Macroeconomic factors. We consider two different macroeconomic factors. The first one is real industrial production growth (IP), which captures fluctuations in the real economy and is available at the monthly frequency. In the classic Fama-MacBeth regression, innovations in IP display a significantly negative risk premium. The three-pass procedure instead finds it insignificant; in addition, IP is effectively uncorrelated with the factors that seem to price returns: the $\mathrm{R}_{g}^{2}$ for IP is about $2 \%$. The three-pass procedure therefore identifies industrial production as essentially a spurious factor.

This can also be seen graphically by looking at the last panel of Figure 5, which reports the cumulated innovations in IP and the version cleaned of measurement error. Most of the variation disappears from the cleaned factor, suggesting that the factor is mostly spurious within our framework.

The same happens for the $L N$ macro factors: standard two-step Fama-MacBeth regression finds a large and statistically significant risk premium for the first factor. However, the three-pass method reveals that that factor is essentially pure noise $\left(\mathrm{R}_{g}^{2}=1 \%\right)$, as are the other three factors. All factors have an insignificant risk premium.

Market frictions. Some of the most interesting results appear with respect to two theoretically motivated non-tradable factors related to market frictions: liquidity and intermediary capital.

By simply running Fama-MacBeth regression, the Pástor and Stambaugh (2003) liquidity factor does not appear to be priced in this cross section of 202 portfolios: its risk premium is $2 \mathrm{bp}$ per month, with a standard error of $97 \mathrm{bp}$. The three-pass analysis shows instead that the liquidity factor commands a statistically significant risk premium of about $26 \mathrm{bp}$ per month.

The prices of the two intermediary factors of He et al. (2016) and Adrian et al. (2014) also vary with the estimation method. Relative to the results obtained using standard Fama-MacBeth regression, 
the three-pass method finds a slightly smaller (but still very large and significant) risk premium for the Adrian et al. (2014) proxy for intermediary capital. The related factor built by He et al. (2016), instead, appears to have a risk premium of zero when estimated via Fama-MacBeth regression, whereas the risk premium appears much larger - 30bp - when estimated using the three-pass method. ${ }^{14}$

Overall, the three-pass procedure shows much stronger support for both types of factors (liquiditybased and intermediary-based) than standard Fama-MacBeth regressions do.

Strength of the factors. We conclude with a remark on the strength of the factors studied. As the last column in Tables 5 and 6 shows, almost all the factors we study are strong factors, according to our Wald test. However, the test also identifies a few weak factors (more precisely, factors for which we cannot reject the null that they are weak), including industrial production growth and one of the three LN factors (the other two are strong but unpriced). The fact that these macro factors are weak in the cross-section of returns is consistent with the low time-series $\mathrm{R}_{g}^{2}$, visible also from Figure 5 .

\subsection{Observable and Unobservable Factors}

The core of our estimation methodology is the link between the observable factors $g_{t}$ and the unobservable factors $v_{t}$, through Equation (2). In particular, $\eta$ represents the loadings of $g_{t}$ onto the $p$ factors, and therefore reveals the exposures of the observable factors to the fundamental priced factors.

In Table 7 we decompose the variance of $g_{t}$ explained by the set of factors $v_{t}$ into the components due to each individual factor (which is possible because factors $\widehat{v}_{t}$ are orthogonal to each other). Each row of the table, therefore, sums up to $100 \%$. This allows us to highlight which fundamental factors are most responsible for the variation of the observable factors. Note that the factors are ordered by their eigenvalues (largest to smallest).

The first row shows that the market return loads mostly onto the first factor, (i.e., on the factor with the largest eigenvalue). This is expected because the market represents the largest source of common variation across assets. The other portfolio-based models (such as FF5 and $H X Z$ ) show interesting variation in the exposure of observable factors to the latent ones. For example, SMB loads on both the first and second factors, HML mostly on the third one, and Momentum almost exclusively on the fourth factor. RMW loads substantially on at least four factors (including the sixth one), and CMA loads mostly on the same factor as HML. However, CMA and HML are still strongly distinguished by a differential exposure to the other factors.

Macro factors load onto these fundamental factors in nontrivial ways. IP is mostly exposed to the sixth factor (to which RMW and CMA are exposed as well). The first $L N$ factor seems exposed uniformly to all risks sources (but its overall risk premium is insignificant because these exposures are small in absolute level, and the factor is very noisy, as explained above).

Finally, both the liquidity factor of Pástor and Stambaugh (2003) and the intermediary factor of He et al. (2016) are strongly exposed to the first latent factor.

\footnotetext{
${ }^{14}$ The economic significance is low for this factor in monthly equity data, as was already pointed out in He et al. (2016); our results here match the results in that paper, which only controls for the market in two-pass regressions.
} 


\subsection{From the Individual Risk Premium to Multifactor Risk Premia}

The three-pass method presented in this paper achieves an estimate of risk premia (and their standard error) associated with each factor by relating each factor in $g_{t}$ individually to the priced latent factors $v_{t}$. At the same time, as discussed in Section 2, the risk premia we estimate can be interpreted as those of a multifactor model in which all observable factors in $g_{t}$ appear directly (together with some additional latent factors). The rotation-invariance result of Section 2 guarantees that this interpretation always holds. Similarly, the standard errors reported in Tables 5 and 6 are the same standard errors as those one would obtain in a two-pass cross-sectional regression using as factors $\widehat{g}_{t}$ and any $(p-d)$ latent factor estimates.

Importantly, this is true even when the factors in $\widehat{g}_{t}$ are highly correlated. For example, the market and liquidity factors both load highly on the first principal component and are therefore highly correlated. One might expect that in a two-pass regression where both factors are included, it would be hard to separately identify the two risk premia. Instead, the two individual risk premia are well identified (as can be seen from the standard errors) because these two factors are simply a rotation of a well-identified model, as implied by the invariance result.

\subsection{Risk Premia across Asset Classes}

The main analysis presented in the previous section has focused on a large cross-section of equity portfolios, for which a long time series is available. In this section we explore risk premia for the same factors discussed above as we look instead at non-equity portfolios.

We obtain from Asaf Manela's website the time series of non-equity portfolio returns used in He et al. (2016), which in turn collects portfolio data from various sources. The data includes ten maturitysorted government bond portfolios, ten corporate bond portfolios sorted on yield spread, six sovereign bond portfolios, 18 S\&P 500 option portfolios sorted on moneyness and maturity, six currency portfolios

sorted on interest rate differentials, six currency portfolios sorted on currency momentum, 24 commodity futures returns, and $20 \mathrm{CDS}$ portfolios sorted by spread, for a total of 100 non-equity portfolios. Due to data availability for the non-equity portfolios, the sample covers the period 1970-2012. In addition, since not all portfolios are available for the entire time period, we adjust the sample size accordingly to estimate the variance and pairwise covariance separately. While the resulting covariance matrix is not necessarily positive-definite, it leads to consistent estimates for PCs.

Table 8 reports the results of the risk premia estimation with our three-pass procedure using equity and non-equity assets. The left panel of the table shows the results using the 202 equity portfolios (as in our main analysis) over this sample period. The results are qualitatively and quantitatively similar to our baseline results that use a longer sample.

The middle panel of the table uses as test assets the 100 non-equity portfolios together with some equity portfolios (the Fama-French 25 portfolios), whereas the right panel uses only the non-equity portfolios. Consistent with our main analysis, we use 6 factors for the cross-section of equity portfolios (with cross-sectional $\mathrm{R}^{2}$ of $63 \%$ ); we instead use a 5 -factor model for the non-equity portfolios, as suggested by our estimator for the number of factors (middle and right panel, with cross-sectional $R^{2} \mathrm{~s}$ 
respectively of $57 \%$ and $53 \%$ ).

The estimates of risk premia over these different groups of test portfolios are surprisingly stable, with few exceptions (like equity momentum). For example, the market risk premium is estimated to be positive and large in the cross-section of non-equity portfolios; similarly, the liquidity factor displays a risk premium of around 20bp per month in all of these samples, and the same goes for the intermediary capital factors. Finally, industrial production is estimated to have a zero risk premium in every case.

This result points to the existence of common risk factors across different markets. Contrary to existing evidence of large segmentation among markets, these results suggest that at least some aggregate risk factors are pervasive across many markets and their risk premia are consistent across them. Key to correctly uncovering the risk premium of these factors is properly controlling for the non-observable factors, which in this paper we achieve using principal component analysis.

\subsection{Robustness and Additional Analysis}

In this section we explore the robustness of our main results to the choice of test portfolios, sample period, and choice of penalty function for recovering the factor space, particularly as it relates to the potential weakness of factors. We also discuss how we handle time variation in risk premia and risk exposures.

\subsubsection{Robustness to the Choice of Test Portfolios}

Our main empirical results are obtained using a large set of 202 portfolios, and our methodology is specifically designed to be used with as many assets as possible, so that all relevant dimensions of risk will be expressed in the cross section. It is natural, however, to wonder to what extent the results are affected by the particular selection of test assets.

To investigate this question systematically, we perform the following robustness exercise. From the 202 test portfolios we use in our empirical exercise, we randomly select (without replacement) half of the test portfolios, and we re-estimate the risk premium of all observable factors in this subsample. ${ }^{15}$ We repeat this exercise 10,000 times, thus obtaining a distribution of risk premia estimates across subsamples of 101 portfolios each, randomly selected.

Figure 7 shows the results for several factors. Note that all panels of the figure report the same range of risk premia (x axis, between -20bp and 100bp), so that the histograms are easily comparable across panels. The results are quite heterogeneous across factors. In the top left panel, we see that the risk premium for the market return is clearly positive in the vast majority of cases (it is below zero only in a small set of subsamples). At the same time, its exact magnitude varies across subsamples. The top right panel shows that instead the risk premia of SMB and HML are much more precisely estimated using our three-pass regression method, and similarly for momentum (middle left panel).

The last three panels show interesting results for non-tradable factors. Confirming the results of Table 6 and Figure 5, IP is a useless factor, with a risk premium of effectively zero across all subsamples. On the contrary, liquidity and intermediary capital factors all appear positively priced across subsamples.

\footnotetext{
${ }^{15}$ We set $\breve{p}=6$, but results are similar for other choices of $\breve{p}$.
} 
Overall, our subsample results show that the conclusions of our empirical analysis are robust to the selection of the test assets.

\subsubsection{Robustness to the Choice of Time Period}

A potential concern when working with principal components is the stability of the estimated loadings and factors over time. While recent econometrics literature has highlighted useful stability properties of principal components analysis (for example, Bates et al. (2013)), the extent to which our risk premia estimates are consistent across time periods is an empirical question that we explore in this section.

Similarly to the robustness with respect to the test assets, we perform our robustness with respect to the sample period by resampling half the time periods randomly without replacement, and looking at the variability of the risk premia estimates. Simple resampling in the time series is possible in our context because of the low serial correlation of returns and factor innovations over time.

Figure 8 shows the results. Both quantitatively and qualitatively, the results are very similar to the ones in the previous section (where we randomly resampled the cross-section as opposed to the time series). The results show that all of the main conclusions of our main analysis hold when looking across subsamples.

While the stability across subsamples may seem surprising, it is useful to note that our risk premia estimator is not only based on PCA. Instead, a key step is the rotation of the factors extracted from PCA into the factor of interest $g_{t}$. So any rotation that makes the extracted factors $v_{t}$ differ across subsamples will be entirely offset by a corresponding rotation of the loading of $g_{t}$ onto those factors, $\eta$ - making the risk premia estimates more stable.

\subsubsection{Weak Factors}

For the invariance result to hold, it is essential that the control factors, together with $g_{t}$, span the entire factor space. The first step of our three-pass procedure involves using principal component analysis to recover the factor space. As we discuss in the paper, our procedure will work even if the observable factor $g_{t}$ is weak (in fact, we propose a test for whether $g_{t}$ is weak), but will not necessarily recover the entire factor space if the underlying latent factors are weak. In this section we summarize our main theoretical and empirical arguments for using PCA in practice, and propose an additional robustness test to exclude that the presence of weak factors is distorting our results.

In theory, weak latent factors - unobservable factors for which the dispersion of risk exposures is small in the cross-section - can affect our estimator because they have low eigenvalues, and PCA might fail to choose them as controls. However, it is useful to point out that for weak factors to bias our estimates of risk premia, they also need to have high risk premia, which allows them to explain a significant portion of the cross-section of average returns. But large risk premia for factors with low eigenvalues imply high Sharpe ratios for these factors. A first theoretical argument in favor of focusing on the PCs with largest eigenvalues are good-deal bounds, which impose a theoretical upper bound on the potential bias from weak factors (see Kozak et al. (2015) for a related discussion).

A second, empirical, argument is that when using PCA, we can easily add additional factors with 
lower and lower eigenvalues, and verify that the risk premia estimates are stable. For example, in Tables 5 and 6 , we explore the robustness of the results using 4,5 and 6 factors. These robustness exercises are theoretically motivated by Theorem 4, which guarantees that adding "too many" principal components does not affect the consistency of our estimator.

A third way to verify that weak factors are not driving our empirical results is the comparison of the risk premia estimated for tradable factors using our three-pass procedure with those obtained as timeseries average of the portfolios' excess returns. As discussed in previous sections, the two should be the same if the factor model is correctly specified. Biases due to the presence of weak latent factors should produce significant differences between the estimates using cross-sectional methods and the time-series averages.

Finally, we propose here an additional robustness test with respect to the possibility of weak factors, based on changing the objective function when extracting the statistical factors from the panel of returns. Recall that the first step towards PCA is to calculate eigenvalues of the covariance matrix of returns, which equal the variances of the corresponding PCs, and that the constructed factors are eigenvectors associated with the largest few eigenvalues.

Since weak factors are factors with low eigenvalues, which however explain the cross-section of returns, we can modify the objective function to account for the contribution to the cross-sectional variation. That is, rather than finding factors that best explain the comovement of stock returns, we find factors that strike a balance between explaining the time-series comovement of stock returns and the cross-sectional variation of expected returns. This alternative objective function was first proposed by Connor and Korajczyk (1986). It is a convenient reference point because it puts equal weight on the two components of the objective function - the time-series and the cross-sectional variation.

More specifically, recall that our PCA formula given in (5) is the solution to the following optimization problem:

$$
\min _{\beta, \bar{V}} n^{-1} T^{-1}\|\bar{R}-\beta \bar{V}\|_{\mathrm{F}}^{2}, \quad \text { subject to } \quad T^{-1} \bar{V} \bar{V}^{\top}=\mathbb{I}_{\widehat{p}}
$$

By our rotation invariance result, it would give the same risk premia estimates if we were to use an alternative normalization $n^{-1} \beta^{\top} \beta=\mathbb{I}_{n}$. Connor and Korajczyk (1986) suggest another optimization problem:

$$
\min _{\beta, \bar{V}, \gamma} n^{-1} T^{-1}\|\bar{R}-\beta \bar{V}\|_{\mathrm{F}}^{2}+w n^{-1}\|\bar{r}-\beta \gamma\|_{\mathrm{F}}^{2}, \quad \text { subject to } \quad n^{-1} \beta^{\top} \beta=\mathbb{I}_{n},
$$

where they choose $w=1$. The solution turns out to be

$$
\tilde{\beta}=n^{1 / 2}\left(\tilde{\varsigma}_{1}: \tilde{\varsigma}_{2}: \ldots: \tilde{\varsigma}_{\tilde{p}}\right), \quad \text { and } \quad \tilde{V}=\left(\tilde{\beta}^{\top} \tilde{\beta}\right)^{-1} \tilde{\beta}^{\top} \bar{R}
$$

where $\tilde{\varsigma}_{1}, \tilde{\varsigma}_{2}, \ldots, \tilde{\varsigma}_{\widehat{p}}$ are the eigenvectors associated with the largest eigenvalues of the matrix $n^{-1} T^{-1} \bar{R} \bar{R}^{\top}+$ $w n^{-1} \bar{r} \bar{r}^{\top}$. Note that starting from CK's formulation, setting $w=0$ (thus focusing entirely on time-series comovement) recovers the standard PCA.

The CK approach can be used instead of the standard PCA in Step (i) of our three-step procedure. 
Since the second term of the objective function is the cross-sectional $R^{2}$, it may help picking latent factors that have large risk premia but are weak. We can then continue Step (ii) and (iii) as in Section 5 using the estimated latent factors together with $g_{t}$ to estimate risk premia. Note that the CK approach does not allow for a free zero-beta rate, so we assume it is equal to the risk free rate instead of estimating it in Step (ii).

In Table 9, we show how the risk premia estimates differ between the baseline PCA (left panel, $w=0$ ) and the Connor-Korajczyk (CK) approach (right panel, $w=1$ ). Because we do not estimate the zero-beta rate in this comparison, the left panel differs from Tables 5 and 6 . Nevertheless, the fact that there is virtually no difference in the risk premia estimates suggests that weak factors are either not present in this dataset we consider, or if they are, they have small enough risk premia that ignoring them has little consequence for our estimates.

Taken together, these considerations lead us to conclude that for the purposes of estimating risk premia, using PCA to recover the factor space represents a simple yet robust solution.

\subsubsection{Portfolios vs. Individual Stocks}

To estimate risk premia, we recommend using characteristic-sorted portfolios instead of individual stocks. The main advantage of using portfolios is that their risk exposures are more stable over time, as discussed at length in the asset pricing literature. This is particularly important in our setting, because we assume the betas of the test assets are constant.

To see this intuition more formally, call $\tilde{r}_{t}$ is the vector of time- $t$ returns for $m$ individual stocks, and $c_{t}$ a $m \times n$ matrix of characteristics (or their functions) observed at time $t$ for the $m$ stocks. The typical procedure to construct characteristic-sorted portfolios in asset pricing categorizes stocks at each time $t-1$ into groups based on one or more observed characteristics, and then obtains the portfolio return at time $t$ using equal or market-value weights for stocks in each group.

The sorting procedure can be represented mathematically by constructing the matrix $c_{t-1}$ stacking side-by-side the $n$ dummy variables corresponding to each characteristic-sorted group. For example, to construct 10 size-based portfolios, $c_{t-1}$ would be an $m \times 10$ matrix containing 10 dummy variables, each indicating the size group in which each stock belongs at time $t-1$. The $n$ characteristic-sorted portfolio returns from $t-1$ to $t$ are simply the coefficients of a cross-sectional regression of $\tilde{r}_{t}$ onto $c_{t-1}$, since $c_{t-1}$ contains only dummies. More generally, given any matrix $c_{t-1}$, the $n$ characteristics-sorted portfolio returns at time $t$ are:

$$
r_{t}=\left(c_{t-1}^{\top} c_{t-1}\right)^{-1} c_{t-1}^{\top} \tilde{r}_{t},
$$

where the term $\left(c_{t-1}^{\top} c_{t-1}\right)^{-1} c_{t-1}$ therefore represents the time- $(t-1)$ portfolio weights.

Using this expression that links $r_{t}$ and $\tilde{r}_{t}$, it is immediate to find that if individual factor exposures are linear functions of $c_{t-1}$ (e.g., Avramov and Chordia (2006)), then the sorted portfolios have constant factor exposures. Specifically, extending our setup to include time-varying factor exposures, ignoring $\alpha$ and zero-beta rate for individual stocks for simplicity, we have:

$$
\tilde{r}_{t}=\beta_{t-1} \gamma+\beta_{t-1} v_{t}+\tilde{u}_{t} .
$$


Now, suppose that $\beta_{t-1}=c_{t-1} \beta$, for some $n \times p$ matrix $\beta$, then characteristics-sorted portfolio returns are given by:

$$
r_{t}=\left(c_{t-1}^{\top} c_{t-1}\right)^{-1} c_{t-1}^{\top} \tilde{r}_{t}=\left(c_{t-1}^{\top} c_{t-1}\right)^{-1} c_{t-1}^{\top}\left(c_{t-1} \beta \gamma+c_{t-1} \beta v_{t}+\tilde{u}_{t}\right)=\beta \gamma+\beta v_{t}+u_{t},
$$

where $u_{t}=\left(c_{t-1}^{\top} c_{t-1}\right)^{-1} c_{t-1}^{\top} \tilde{u}_{t}$. Therefore, our methodology to estimate risk premia can be applied even if individual stock risk exposures are time-varying, as long as characteristic-sorted portfolios that have constant factor exposures are used as test assets.

In this paper, we take the portfolio-formation step as given, and use characteristic-sorted portfolios that have been proposed in the literature. In contrast, Kelly et al. (2017) construct such portfolios using characteristics and individual stocks for a model specification test. Their results show that PCs based on such portfolios explain more cross-sectional variations than those based on individual stocks, which is consistent with the formal result shown above that characteristic-sorted portfolios will have constant betas if the characteristics are chosen appropriately. Clarke (2015) proposes a related but different method to recover the factor space accounting for characteristics. His method estimates the same cross-sectional regression of returns on characteristics as equation (9), and then creates portfolio sorts based on the fitted returns. This approach, however, does not produce constant-beta portfolios in our setting.

\subsubsection{Time-Varying Risk Premia}

Last but not least, our approach is also applicable to models that allow for time-varying risk premia in addition to time-varying exposures. In fact, the above portfolio formation approach leads to

$$
r_{t}=\beta \gamma_{t-1}+\beta v_{t}+u_{t}
$$

where $\gamma_{t-1}$ is the risk premia vector. It is straightforward to rewrite this model as

$$
r_{t}=\beta \mathrm{E}\left(\gamma_{t-1}\right)+\beta\left(v_{t}+\gamma_{t-1}-\mathrm{E}\left(\gamma_{t-1}\right)\right)+u_{t}
$$

where $\tilde{v}_{t}=v_{t}+\gamma_{t-1}-\mathrm{E}\left(\gamma_{t-1}\right)$ serves as a new factor that has a zero unconditional mean. Therefore, we can interpret the estimated risk premia as estimates of their time-series average, i.e., $\mathrm{E}\left(\gamma_{t-1}\right)$, in the above empirical analysis.

\section{Conclusion}

We propose a three-pass methodology to estimate the risk premium of observable factors in a linear asset pricing model, that is consistent even when not all factors in the model are specified and observed. The methodology relies on a simple invariance result that states that to correct the omitted variable problem in cases where not all factors are observed, it is sufficient to control for enough factors to span the entire factor space when running cross-sectional regressions. In these cases, the risk premium for observable factors will be consistent even though the risk exposures cannot be identified. We propose 
to employ PCA to recover the factor space and effectively use the PCs as controls in the cross-sectional regressions together with the observable factors.

Equally important to what we can recover is what we cannot recover if some factors are omitted: how the pricing kernel loads onto the observed factors, as well as the set of true risk exposures to each factor. These can only be pinned down under much stronger assumptions - by identifying all the factors that drive the pricing kernel, and explicitly specifying how they enter the pricing kernel. Instead, a notable property of factor risk premia is precisely that they can be recovered even without specifying all factors, and this is what we focus on in this paper.

The main advantage of our methodology is that it provides a systematic way to tackle the concern that the model predicted by theory is misspecified because of omitted factors. Rather than relying on arbitrarily chosen "control" factors or computing risk premia only on subsets of the test assets, our methodology utilizes the large dimension of testing assets available to control for omitted factors in the cross-sectional regression. It also explicitly takes into account the possibility of measurement error in any observed factor.

Application of the methodology to workhorse factor models using equity test assets yields several compelling results. Contrary to most existing estimates, we find that the risk premium estimate associated with market risk exposure is positive and significant, and close to the time-series average excess return of the market portfolio. This confirms that our methodology correctly recovers the risk premium of the market (and similar results hold for most other tradable factors), thus mitigating misspecification concerns. The most interesting results appear for non-tradable factors. Many standard macroeconomic factors appear insignificant, whereas non-tradable factors related to various market frictions (like liquidity and intermediary leverage) appear strongly significant when considered as part of richer linear pricing models that include additional factors. Similar results hold when looking across asset classes; the stability of the risk premia estimates across markets suggests the presence of pervasive aggregate risks that can be detected once factors specific to the various asset classes are properly accounted for which in this case is achieved using the three-pass methodology we propose.

\section{References}

Adrian, T., E. Etula, and T. Muir (2014). Financial intermediaries and the cross-section of asset returns. The Journal of Finance 69(6), 2557-2596.

Ahn, S. C. and A. R. Horenstein (2013). Eigenvalue ratio test for the number of factors. Econometrica 81, $1203-1227$.

Asness, C. S., A. Frazzini, and L. H. Pedersen (2013). Quality Minus Junk. Technical report, AQR.

Avramov, D. and T. Chordia (2006). Asset pricing models and financial market anomalies. Review of Financial Studies 19(3), 1001-1040.

Bai, J. (2003). Inferential Theory for Factor Models of Large Dimensions. Econometrica 71(1), 135-171.

Bai, J. (2009). Panel Data Models With Interactive Fixed Effects. Econometrica 7r7(4), 1229-1279.

Bai, J. and Y. Liao (2013). Statistical inferences using large estimated covariances for panel data and factor models. Technical report, Columbia University. 
Bai, J. and S. Ng (2002). Determining the number of factors in approximate factor models. Econometrica 70, 191-221.

Bai, J. and S. Ng (2006a, July). Confidence Intervals for Diffusion Index Forecasts and Inference for Factor-Augmented Regressions. Econometrica 74(4), 1133-1150.

Bai, J. and S. Ng (2006b). Evaluating latent and observed factors in macroeconomics and finance. Journal of Econometrics 131(1), 507-537.

Bai, J. and S. Ng (2008). Forecasting economic time series using targeted predictors. Journal of Econometrics 146(2), 304-317.

Bai, J. and G. Zhou (2015). Fama-MacBeth two-pass regressions: Improving risk premia estimates. Finance Research Letters 15, 31-40.

Bai, Z. and J. W. Silverstein (2009). Spectral Analysis of Large Dimensional Random Matrices. Springer.

Balduzzi, P. and C. Robotti (2008). Mimicking portfolios, economic risk premia, and tests of multi-beta models. Journal of Business 8 Economic Statistics 26, 354-368.

Bates, B. J., M. Plagborg-Møller, J. H. Stock, and M. W. Watson (2013). Consistent factor estimation in dynamic factor models with structural instability. Journal of Econometrics 17r7(2), 289-304.

Bernanke, B. S. and K. Kuttner (2005). What explains the stock market's reaction to federal reserve policy. The Journal of Finance 60, 1221-1257.

Black, F., M. C. Jensen, and M. Scholes (1972). The Capital Asset Pricing Model: Some Empirical Tests. In Studies in the Theory of Capital Markets. Praeger.

Bryzgalova, S. (2015). Spurious Factors in Linear Asset Pricing Models. Technical report, Stanford University.

Burnside, C. (2016). Identification and inference in linear stochastic discount factor models with excess returns. Journal of Financial Econometrics 14(2), 295-330.

Chamberlain, G. and M. Rothschild (1983). Arbitrage, factor structure, and mean-variance analysis on large asset markets. Econometrica 51, 1281-1304.

Clarke, C. (2015). The level, slope and curve factor model for stocks. Technical report, University of Connecticut.

Cochrane, J. H. (2009). Asset Pricing:(Revised Edition). Princeton university press.

Connor, G., M. Hagmann, and O. Linton (2012). Efficient semiparametric estimation of the fama-french model and extensions. Econometrica 80(2), 713-754.

Connor, G. and R. A. Korajczyk (1986). Performance measurement with the arbitrage pricing theory: A new framework for analysis. Journal of Financial Economics 15(3), 373-394.

Connor, G. and R. A. Korajczyk (1988). Risk and return in an equilibrium APT: Application of a new test methodology. Journal of Financial Economics 21(2), 255-289.

Donoho, D. L. (2000). High-dimensional data analysis: The curses and blessings of dimensionality. Technical report, AMS Math Challenges Lecture.

Fama, E. F. and K. R. French (1993). Common risk factors in the returns on stocks and bonds. Journal of Financial Economics 33(1), 3-56.

Fama, E. F. and K. R. French (2015). A five-factor asset pricing model. Journal of Financial Economics 116(1), 1-22. 
Fama, E. F. and J. D. Macbeth (1973). Risk, Return, and Equilibrium: Empirical Tests. Journal of Political Economy 81(3), 607-636.

Fan, J., Y. Liao, and M. Mincheva (2011). High-dimensional covariance matrix estimation in approximate factor models. Annals of Statistics 39(6), 3320-3356.

Fan, J., Y. Liao, and M. Mincheva (2013). Large covariance estimation by thresholding principal orthogonal complements. Journal of the Royal Statistical Society, B 75, 603-680.

Fan, J., Y. Liao, and J. Yao (2015). Power enhancement in high-dimensional cross-sectional tests. Econometrica 83(4), 1497-1541.

Ferson, W. E. and C. R. Harvey (1991). The variation of economic risk premiums. Journal of Political Economy 99(2), 385-415.

Forni, M. and L. Reichlin (1998, July). Let's Get Real: A Factor Analytical Approach to Disaggregated Business Cycle Dynamics. The Review of Economic Studies 65(3), 453-473.

Frazzini, A. and L. H. Pedersen (2014). Betting against beta. Journal of Financial Economics 111(1), $1-25$.

Gagliardini, P., E. Ossola, and O. Scaillet (2016). Time-varying risk premium in large cross-sectional equity datasets. Econometrica 84(3), 985-1046.

Gibbons, M., S. A. Ross, and J. Shanken (1989). A test of the efficiency of a given portfolio. Econometrica 57(5), 1121-1152.

Gospodinov, N., R. Kan, and C. Robotti (2013). Chi-squared tests for evaluation and comparison of asset pricing models. Journal of Econometrics 173(1), 108-125.

Gospodinov, N., R. Kan, and C. Robotti (2014). Misspecification-Robust Inference in Linear AssetPricing Models with Irrelevant Risk Factors. The Review of Financial Studies 27(7), 2139-2170.

Gospodinov, N., R. Kan, and C. Robotti (2016). Spurious inference in reduced-rank asset-pricing models. Technical report, Imperial College London.

Harvey, C. R., Y. Liu, and H. Zhu (2016). ...and the Cross-Section of Expected Returns. The Review of Financial Studies 29(1), 5-68.

He, Z., B. Kelly, and A. Manela (2016). Intermediary asset pricing: New evidence from many asset classes. Technical report, National Bureau of Economic Research.

Horn, R. A. and C. R. Johnson (2013). Matrix Analysis (Second ed.). Cambridge University Press.

Hou, K. and R. Kimmel (2006). On the estimation of risk premia in linear factor models. Technical report, Working Paper, Ohio State University.

Hou, K., C. Xue, and L. Zhang (2015). Digesting anomalies: An investment approach. Review of Financial Studies, Forthcoming 28(3), 650-705.

Huberman, G., S. Kandel, and R. F. Stambaugh (1987). Mimicking portfolios and exact arbitrage pricing. The Journal of Finance 42(1), 1-9.

Jagannathan, R., G. Skoulakis, and Z. Wang (2010). The analysis of the cross section of security returns. Handbook of financial econometrics 2, 73-134.

Jagannathan, R. and Z. Wang (1996). The Conditional CAPM and the Cross-Section of Expected Returns. The Journal of Finance 51(1), 3-53. 
Jagannathan, R. and Z. Wang (1998). An asymptotic theory for estimating beta-pricing models using cross-sectional regression. The Journal of Finance 53(4), 1285-1309.

Kan, R. and C. Robotti (2008). Specification tests of asset pricing models using excess returns. Journal of Empirical Finance 15(5), 816-838.

Kan, R. and C. Robotti (2009). Model comparison using the hansen-jagannathan distance. Review of Financial Studies 22(9), 3449-3490.

Kan, R. and C. Robotti (2012). Evaluation of Asset Pricing Models Using Two-Pass Cross-Sectional Regressions. In Handbook of Computational Finance, pp. 223-251. Springer.

Kan, R., C. Robotti, and J. Shanken (2013). Pricing model performance and the two-pass cross-sectional regression methodology. The Journal of Finance 68(6), 2617-2649.

Kan, R. and C. Zhang (1999a). GMM tests of stochastic discount factor models with useless factors. Journal of Financial Economics 54(1), 103-127.

Kan, R. and C. Zhang (1999b). Two-Pass Tests of Asset Pricing Models. The Journal of Finance LIV(1), 203-235.

Kelly, B. and S. Pruitt (2013). Market expectations in the cross-section of present values. The Journal of Finance 68(5), 1721-1756.

Kelly, B. and S. Pruitt (2015). The three-pass regression filter: A new approach to forecasting using many predictors. Journal of Econometrics 186(2), 294-316.

Kelly, B., S. Pruitt, and Y. Su (2017). Characteristics are risk exposures. Technical report, University of Chicago.

Kleibergen, F. (2009). Tests of risk premia in linear factor models. Journal of Econometrics 149(2), 149-173.

Kleibergen, F. and Z. Zhan (2014). Mimicking portfolios of macroeconomic factors. Technical report, Brown University Working Paper.

Kozak, S., S. Nagel, and S. Santosh (2015). Interpreting factor models. Technical report, University of Michigan.

Lehmann, B. N. and D. M. Modest (1988). The empirical foundations of the arbitrage pricing theory. Journal of Financial Economics 21(2), 213-254.

Lettau, M. and S. Ludvigson (2001). Resurrecting the (c)capm: A cross-sectional test when risk premia are time-varying. Journal of Political Economy 109(6), 1238-1287.

Lewellen, J. and S. Nagel (2006). The conditional CAPM does not explain asset-pricing anomalies. Journal of Financial Economics 82(2), 289-314.

Lewellen, J., S. Nagel, and J. Shanken (2010). A skeptical appraisal of asset pricing tests. Journal of Financial Economics 96(2), 175-194.

Lucca, D. O. and E. Moench (2015). The pre-FOMC announcement drift. The Journal of Finance 70(1), 329-371.

Ludvigson, S. C. and S. Ng (2009). A factor analysis of bond risk premia. Technical report, National Bureau of Economic Research.

MacKinlay, A. C. and M. P. Richardson (1991). Using Generalized Method of Moments to Test MeanVariance Efficiency. The Journal of Finance 46(2), 511-527. 
McLean, R. D. and J. Pontiff (2015). Does Academic Research Destroy Stock Return Predictability? The Journal of Finance LXXI(1), 1-48.

Moon, H. R. and M. Weidner (2015). Linear regression for panel with unknown number of factors as interactive fixed effects. Econometrica 83(4), 1543-1579.

Moskowitz, T. J. (2003). An analysis of covariance risk and pricing anomalies. Review of Financial Studies 16(2), 417-457.

Newey, W. K. and K. D. West (1987). A simple, positive semi-definite, heteroskedasticity and autocorrelation consistent covariance matrix. Econometrica 55, 703-708.

Onatski, A. (2010). Determining the number of factors from empirical distribution of eigenvalues. Review of Economics and Statistics 92, 1004-1016.

Onatski, A. (2012). Asymptotics of the principal components estimator of large factor models with weakly influential factors. Journal of Econometrics 168, 244-258.

Pástor, L. and R. F. Stambaugh (2003). Liquidity risk and expected stock returns. Journal of Political Economy $111(3), 642-685$.

Pesaran, M. and T. Yamagata (2012). Testing CAPM with a large number of assets. Technical report, University of South California.

Pukthuanthong, K. and R. Roll (2014). A protocol for factor identification. Technical report, UCLA.

Raponi, V., C. Robotti, and P. Zaffaroni (2016). Ex-Post Risk Premia and Tests of Multi-Beta Models in Large Cross-Sections. Technical report, Imperial College London.

Roll, R. (1977). A critique of the asset pricing theory's tests part i: On past and potential testability of the thoery. Journal of Financial Economics 4, 129-176.

Roll, R. and S. A. Ross (1980). An empirical investigation of the arbitrage pricing theory. The Journal of Finance 35(5), 1073-1103.

Ross, S. A. (1976). The Arbitrage Theory of Capital Asset Pricing. Journal of Economics Theory 13, $341-360$.

Shanken, J. (1992). On the Estimation of Beta Pricing Models. The Review of Financial Studies 5(1), $1-33$.

Shanken, J. (1996). Statistical methods in tests of portfolio efficiency: A synthesis. Handbook of statistics 14, 693-711.

Shanken, J. and G. Zhou (2007). Estimating and testing beta pricing models: Alternative methods and their performance in simulations. Journal of Financial Economics 84(1), 40-86.

Stock, J. H. and M. W. Watson (2002a). Forecasting Using Principal Components from a Large Number of Predictors. Journal of the American Statistical Association 97(460), 1167-1179.

Stock, J. H. and M. W. Watson (2002b). Macroeconomic Forecasting Using Diffusion Indexes. Journal of Business 85 Economic Statistics 20(2), 147-162.

Tao, T. (2012). Topics in Random Matrix Theory, Volume 132 of Graduate Studies in Mathematics. American Mathematical Society.

Welch, I. (2008). The link between fama-french time-series tests and fama-macbeth cross-sectional tests. Technical report, UCLA.

White, H. (2000). Asymptotic Theory for Econometricians: Revised Edition. Emerald Group Publishing Limited. 


\section{Figures and Tables}

Figure 1: Histograms of the Standardized Estimates in Simulations
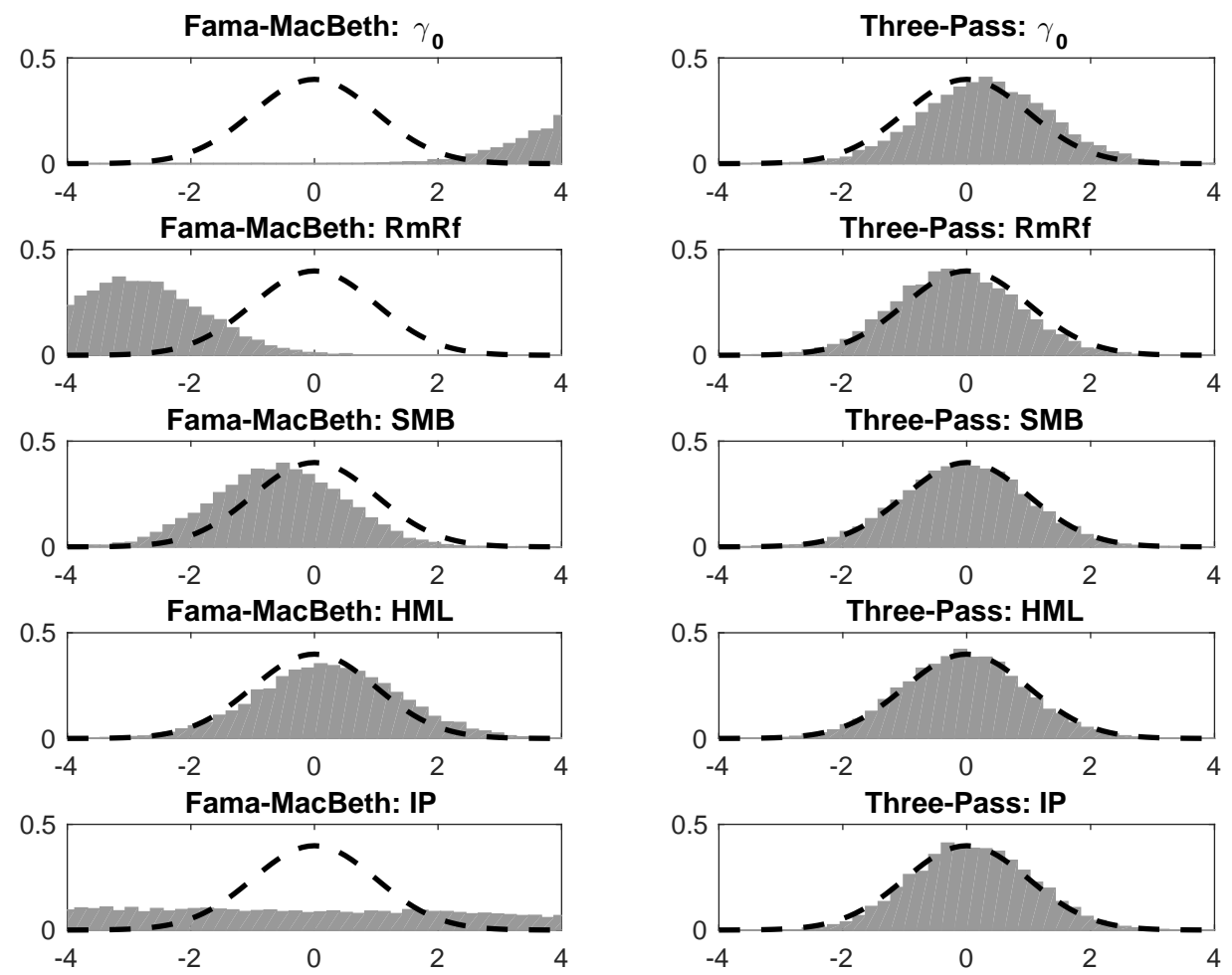

Note: The left panels provide the histograms of the standardized two-pass risk premia estimates using the FamaMacBeth approach for standard error estimation, whereas the right panels provide the histograms of the standardized three-pass estimates using asymptotic standard errors. We simulate the models with $n=200$ and $T=600$. 
Figure 2: Size and Power of the Test Statistic
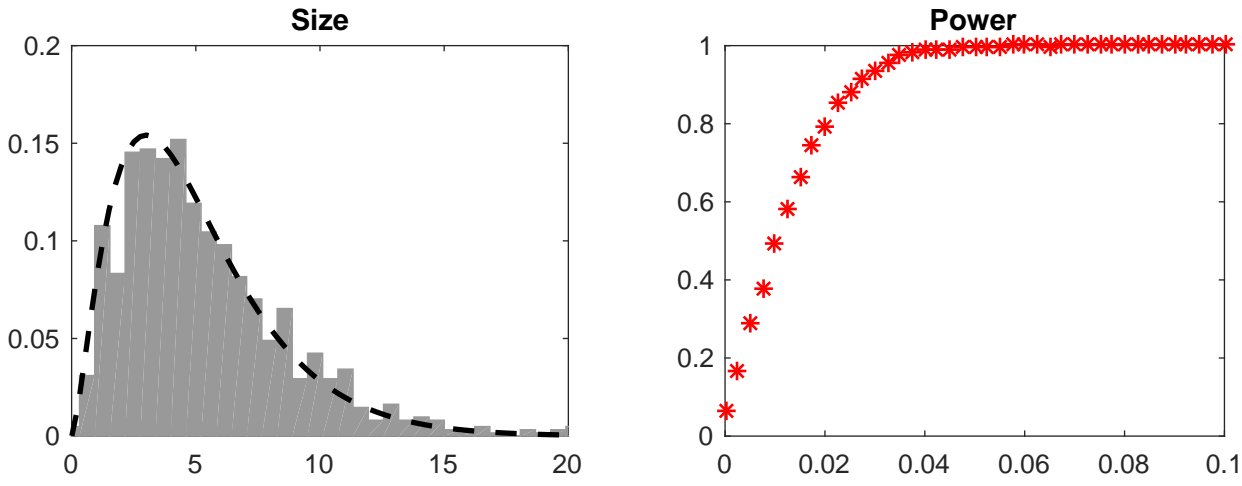

Note: The left panel provides the histogram of the standardized test statistic under the null hypothesis $\eta=0$ along with the density of the chi-squared distribution with 5 degrees of freedom, whereas the right panel plots the rejection probability (y-axis) against $\mathrm{R}_{g}^{2}$ (x-axis). We fix $n=200$ and $T=600$.

Figure 3: First Eight Eigenvalues of the Covariance Matrix of 202 Equity Portfolios
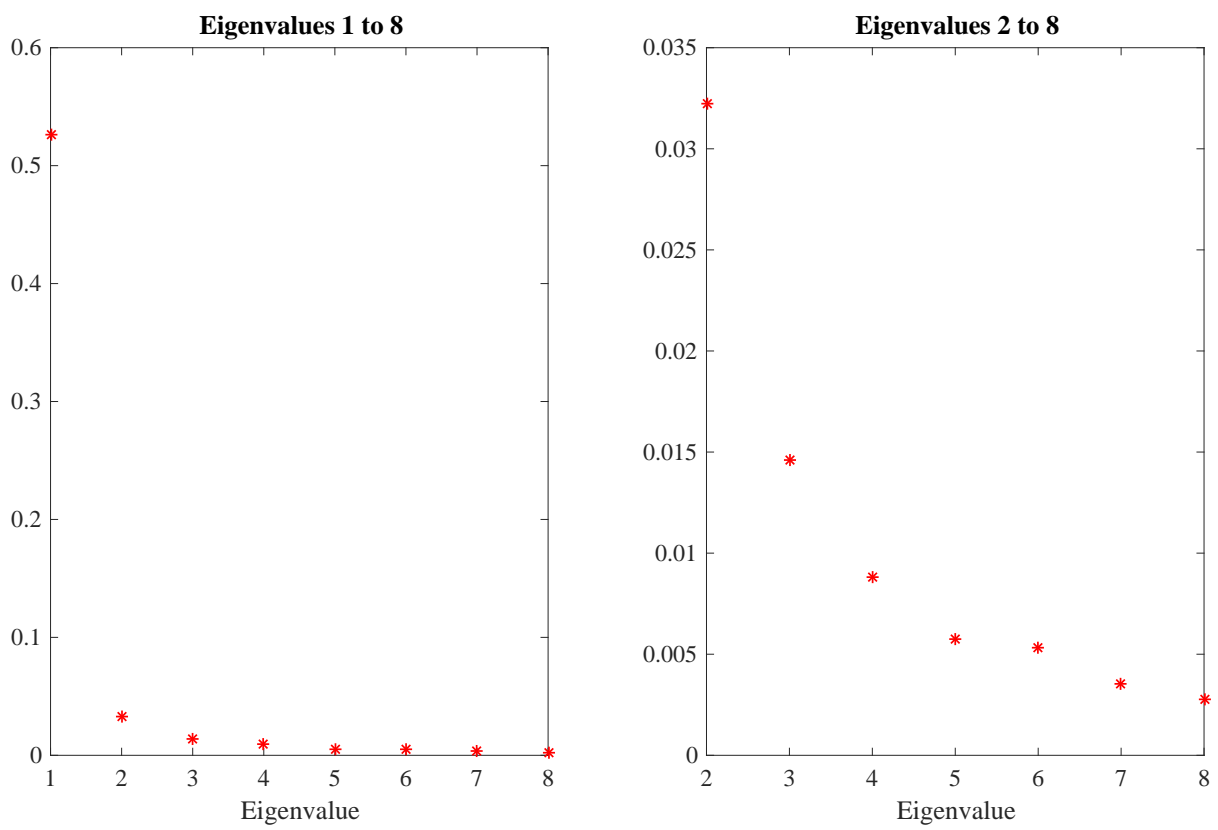

Note: The left panel reports the first eight eigenvalues of the covariance matrix of our 202 test portfolios. The right panel zooms in to the eigenvalues two through eight. 
Figure 4: Predicted and Realized Average Excess Returns in a Six-Factor Model

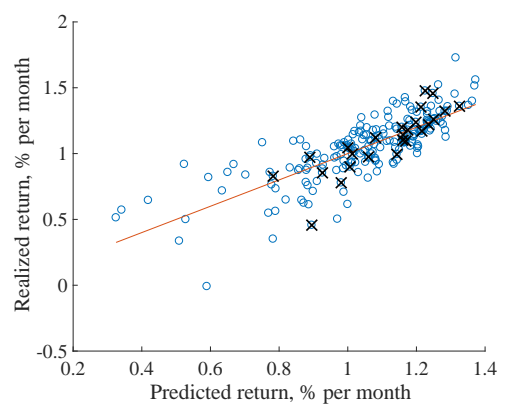

(a) ME and BE/ME-sorted

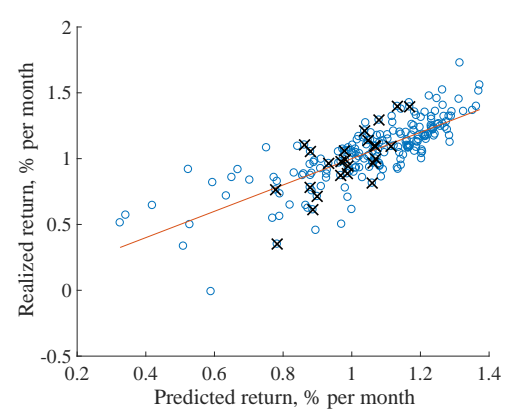

(c) OP and INV-sorted

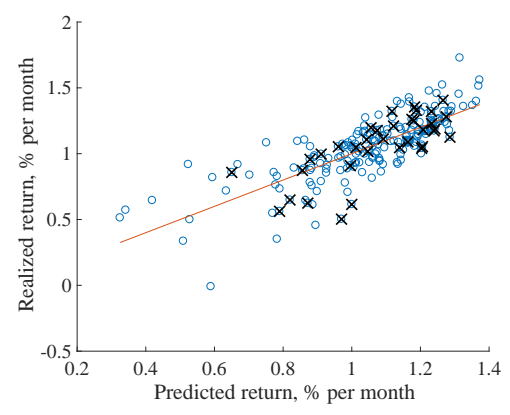

(e) ME and Net Issuance-sorted

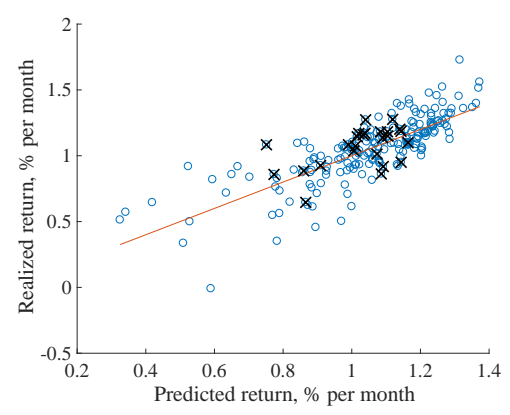

(g) ME and Accruals-sorted

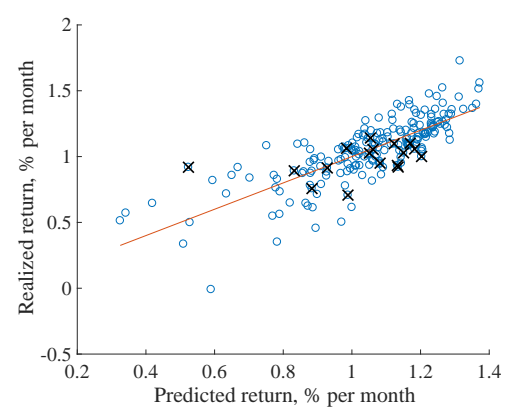

(b) Industry-sorted

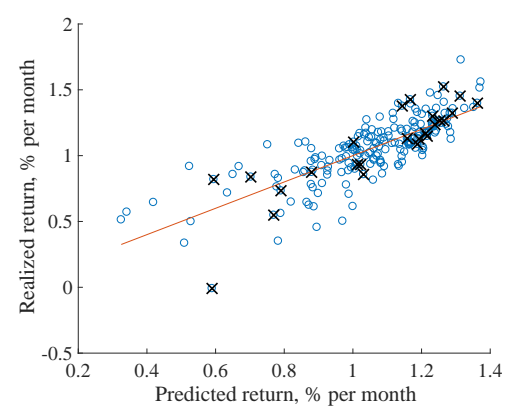

(d) ME and Variance-sorted

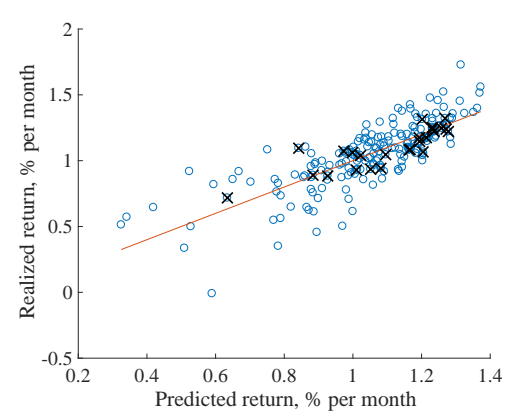

(f) ME and Beta-sorted

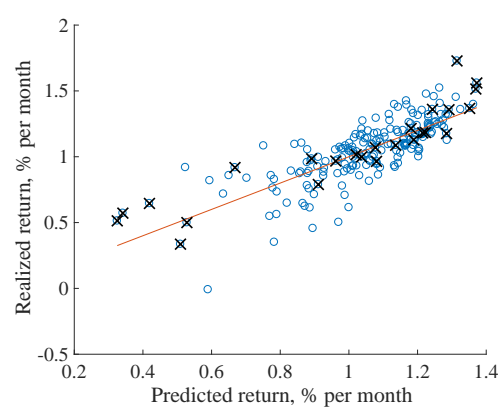

(h) ME and Momentum

Note: This figure reports the predicted average excess returns of the 202 test portfolios against the realized average excess returns. Each panel highlights a different set of test assets. The solid line is the 45-degree line. 
Figure 5: Cumulative Factor Time Series with and without Measurement Error
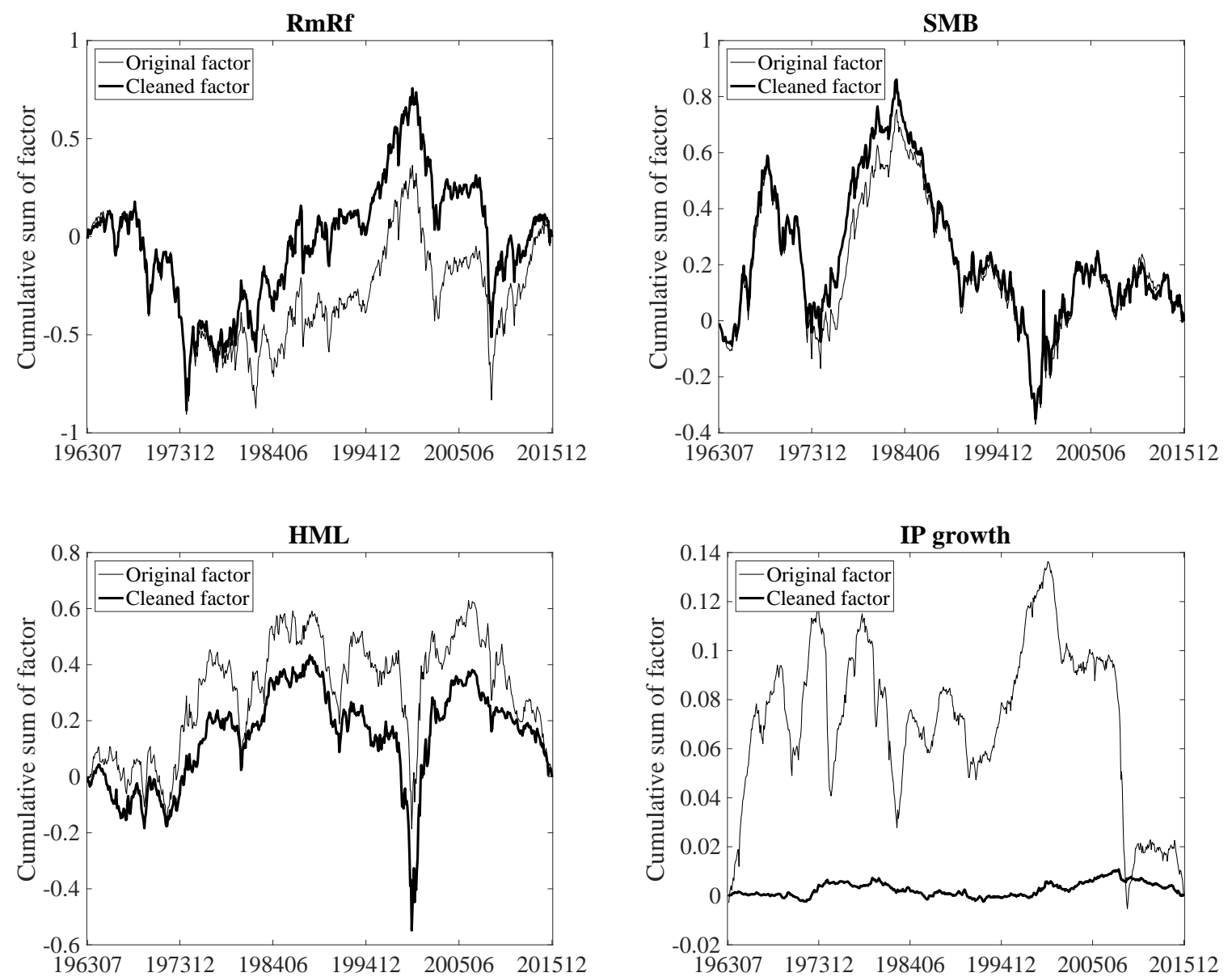

Note: This figure reports the time series of cumulative factor innovations for RmRf, SMB, HML, and IP (thin line) together with the time series obtained from removing measurement error from the factor (thick line). 


\section{Figure 6: Market beta and expected return}

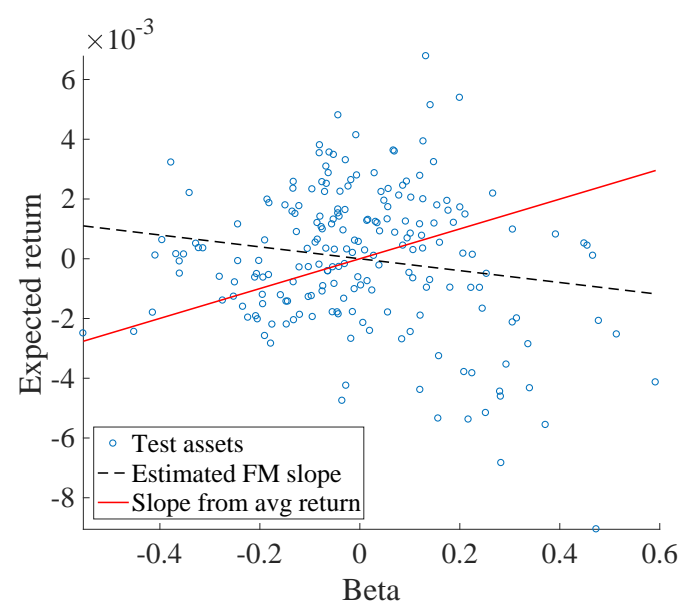

(a) Fama-MacBeth

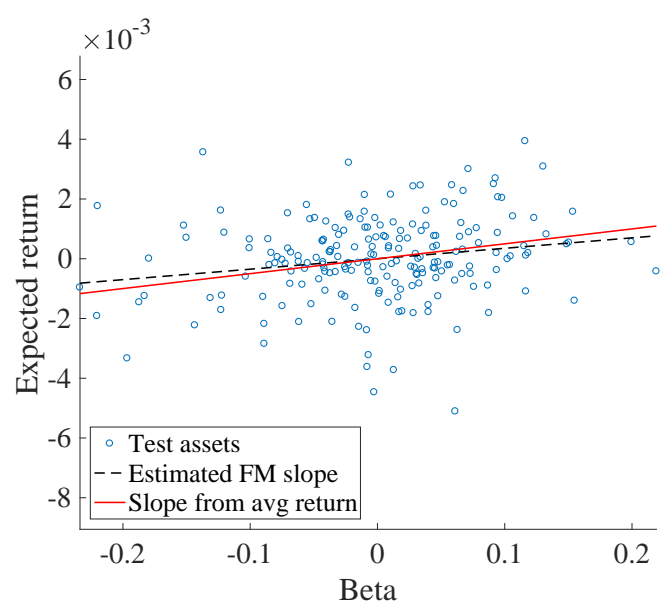

(b) Three-pass estimator

Note: This figure plots expected returns against market beta after partialing out the components explained by the other factors (using residual regression approach). The left panel uses standard Fama-MacBeth with the Fama-French three-factor model. The right panel uses our three-pass regression approach. In each graph, the solid red line corresponds to the market risk premium estimate obtained from the time-series average return of the market portfolio; the dashed line is the Fama-MacBeth slope. If the model is correctly specified, the two lines should coincide. 
Figure 7: Robustness to the Set of Test Portfolios: Resampling Exercise
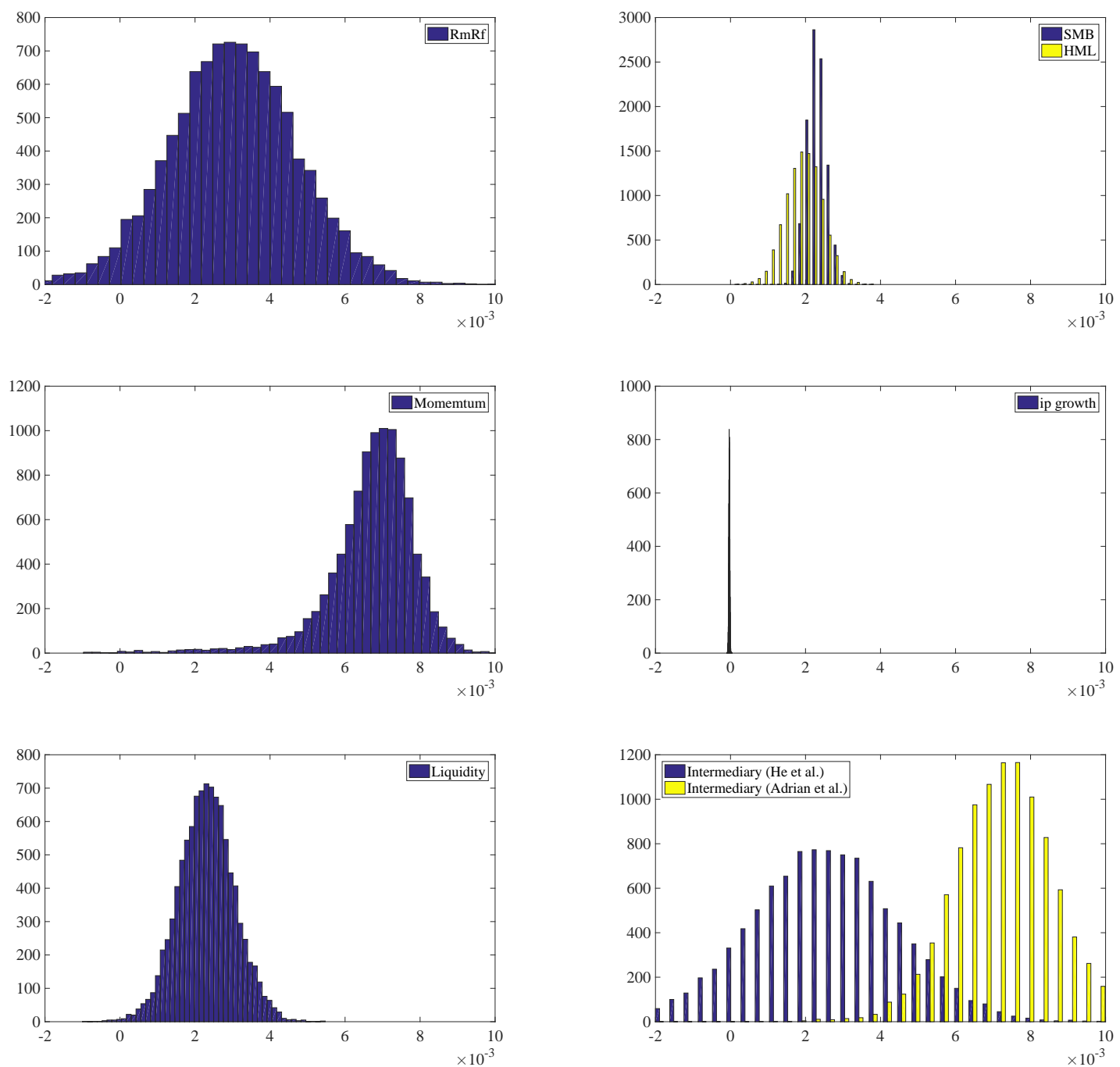

Note: This figure reports the histograms of risk premia estimated using the three-pass estimator across subsamples of the set of 202 test portfolios. We generate 10,000 subsamples by randomly drawing (without replacement) half of the portfolios from the baseline set of 202 portfolios. In each sample we estimate the risk premium of each factor using the three-pass estimator, setting $\breve{p}=6$. The histogram reports the frequency of the risk premia estimates across samples. All figures report the same range for the risk premium, between -20bp and 100bp per month. 
Figure 8: Robustness to the Time Period: Resampling Exercise
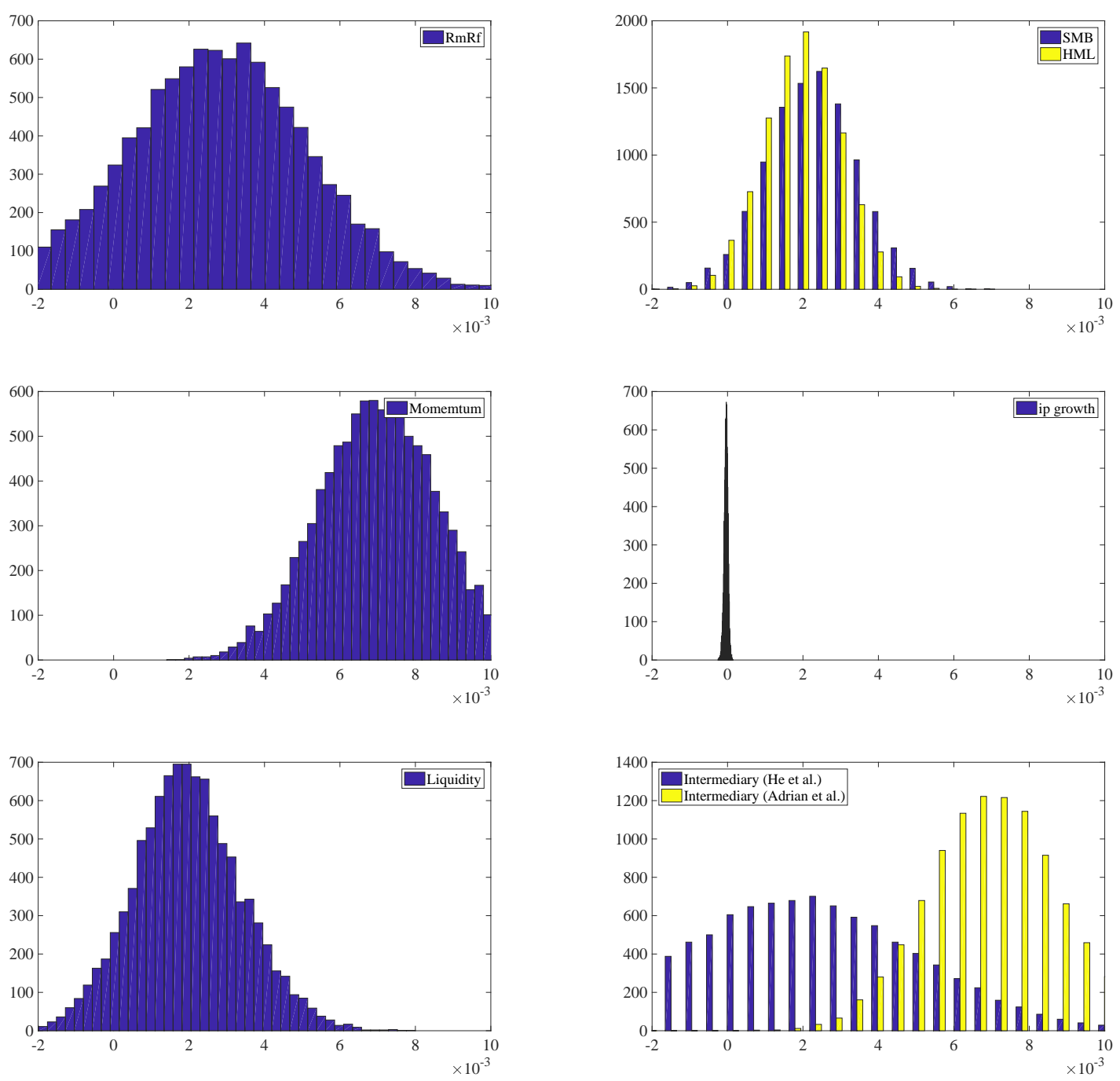

Note: This figure reports the histograms of risk premia estimated using the three-pass estimator across subsamples of the time period. We generate 10,000 subsamples by randomly drawing (without replacement) half of the available time periods (using all of the portfolios available in the selected periods). In each sample we estimate the risk premium of each factor using the three-pass estimator, setting $\breve{p}=6$. The histogram reports the frequency of the risk premia estimates across samples. All figures report the same range for the risk premium, between $-20 \mathrm{bp}$ and $100 \mathrm{bp}$ per month. 
Table 1: Simulation Results for $n=50$

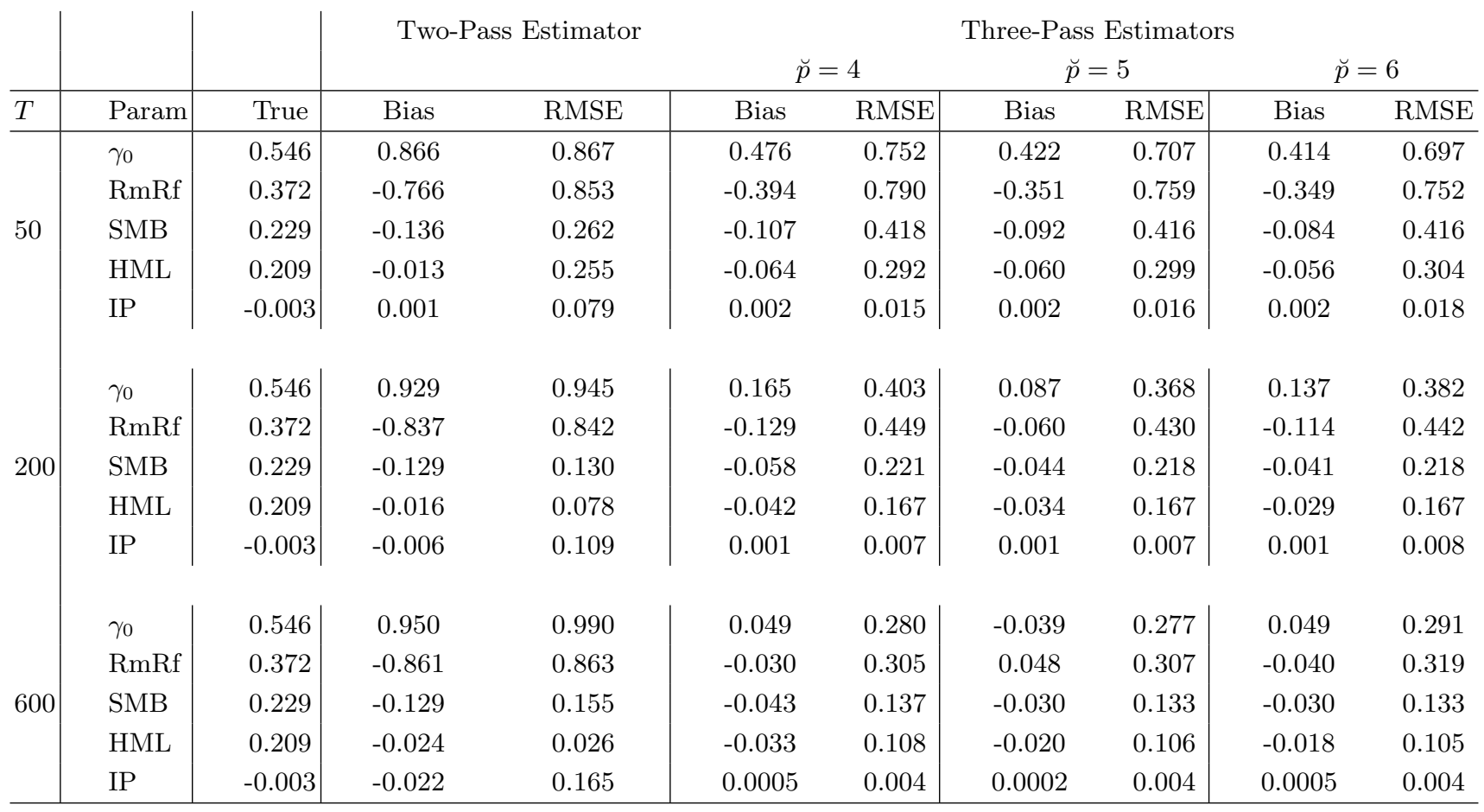

Note: In this table, we report the bias (Column "Bias") and the root-mean-square error (Column "RMSE") of the zero-beta rate and risk premia estimates using two-pass and three-pass estimators with $\breve{p}=4,5$, and 6 , for $n=50$, and $T=50,200$, and 600, respectively. The true data-generating process has five factors, and the parameters are calibrated based on the de-noised five Fama-French factors (RmRf, SMB, HML, RMW, and CMA). The true zero-beta rate is 0.546, and the true risk premia of four noisy yet observed factors (RmRf, SMB, HML, and IP) are provided in the "True" column. All numbers are in percentages. 
Table 2: Simulation Results for $n=100$

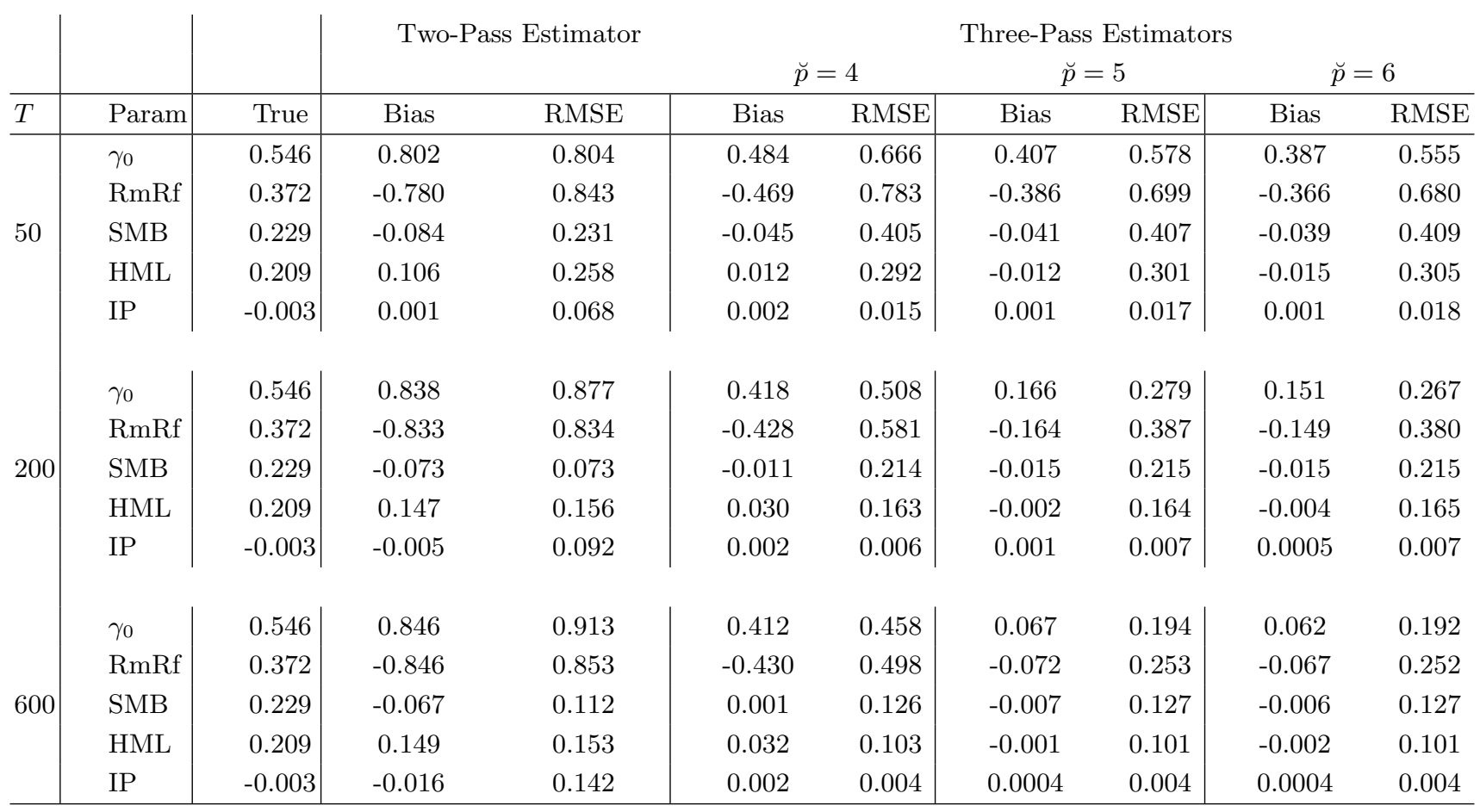

Note: In this table, we report the bias (Column "Bias") and the root-mean-square error (Column "RMSE") of the zero-beta rate and risk premia estimates using two-pass and three-pass estimators with $\breve{p}=4,5$, and 6 , for $n=100$, and $T=50,200$, and 600, respectively. The true data-generating process has five factors, and the parameters are calibrated based on the de-noised five Fama-French factors (RmRf, SMB, HML, RMW, and CMA). The true zero-beta rate is 0.546 , and the true risk premia of four noisy yet observed factors (RmRf, SMB, HML, and IP) are provided in the "True" column. All numbers are in percentages. 
Table 3: Simulation Results for $n=200$

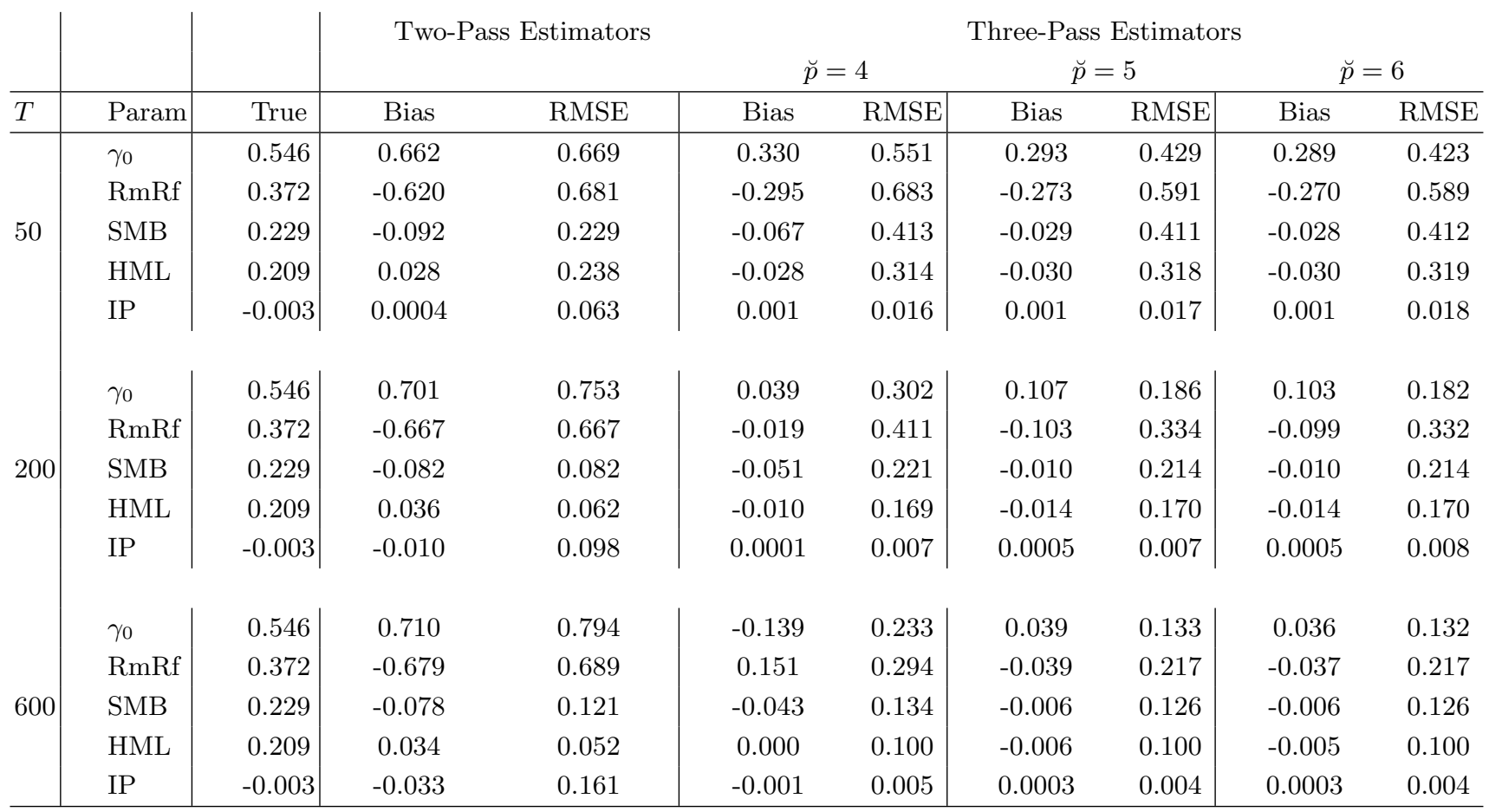

Note: In this table, we report the bias (Column "Bias") and the root-mean-square error (Column "RMSE") of the zero-beta rate and risk premia estimates using two-pass and three-pass estimators with $\breve{p}=4,5$, and 6 , for $n=200$, and $T=50,200$, and 600, respectively. The true data-generating process has five factors, and the parameters are calibrated based on the de-noised five Fama-French factors (RmRf, SMB, HML, RMW, and CMA). The true zero-beta rate is 0.546 , and the true risk premia of four noisy yet observed factors (RmRf, SMB, HML, and IP) are provided in the "True" column. All numbers are in percentages.

Table 4: Simulation Results for the Number of Factors

\begin{tabular}{l|cccccc} 
& \multicolumn{2}{|c}{$n=50$} & \multicolumn{2}{c}{$n=100$} & \multicolumn{2}{c}{$n=200$} \\
$T$ & Median & Stderr & Median & Stderr & Median & Stderr \\
\hline 50 & 3 & 0.66 & 3 & 0.53 & 5 & 0.79 \\
200 & 3 & 0.64 & 4 & 0.83 & 5 & 0.14 \\
600 & 4 & 0.50 & 5 & 0.40 & 5 & 0.40 \\
\hline
\end{tabular}

Note: In this table, we report the median (Column "Median") and the standard error (Column "Stderr") of the estimates for the number of factors. The true number of factors in the data generating process is five. 


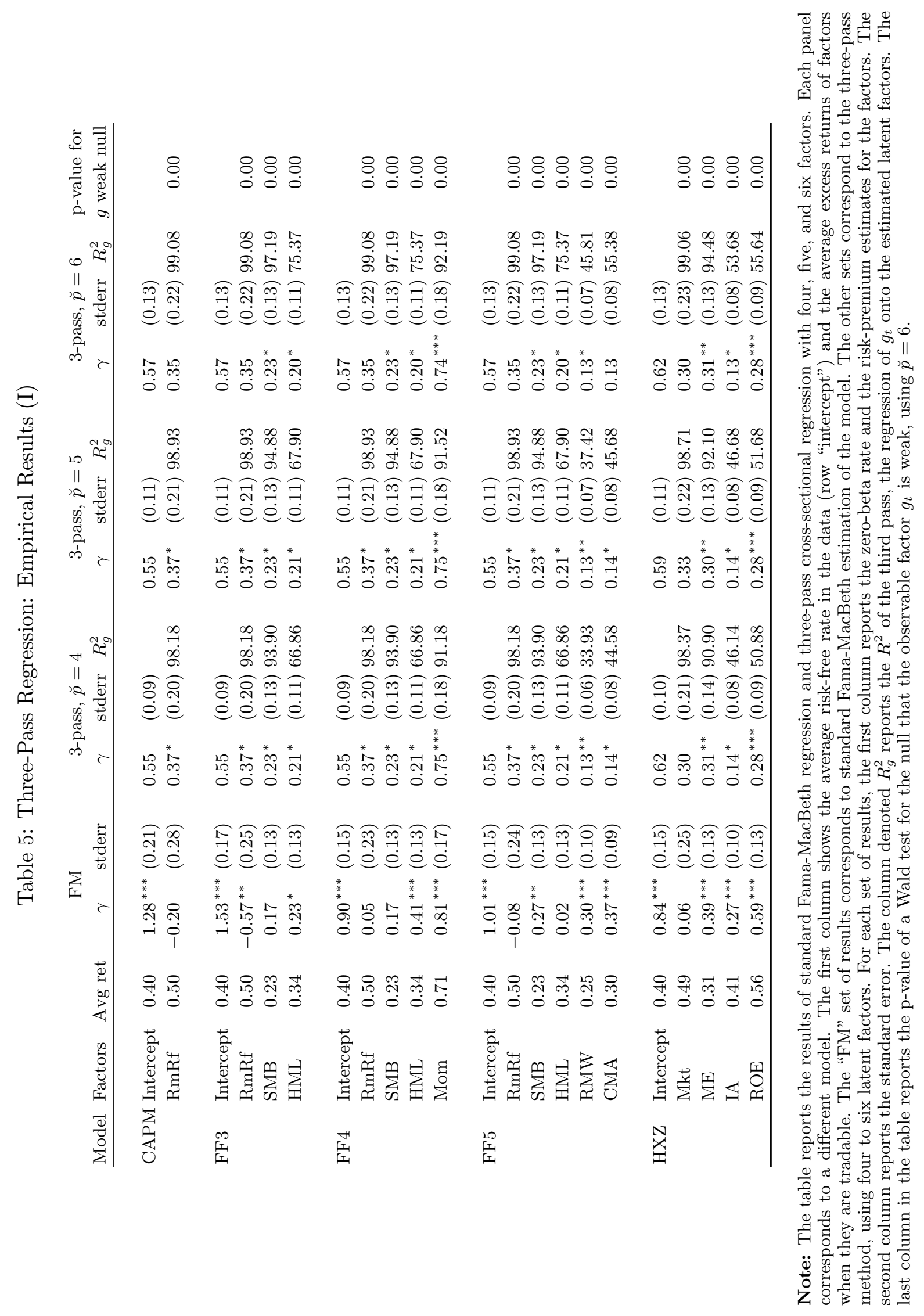




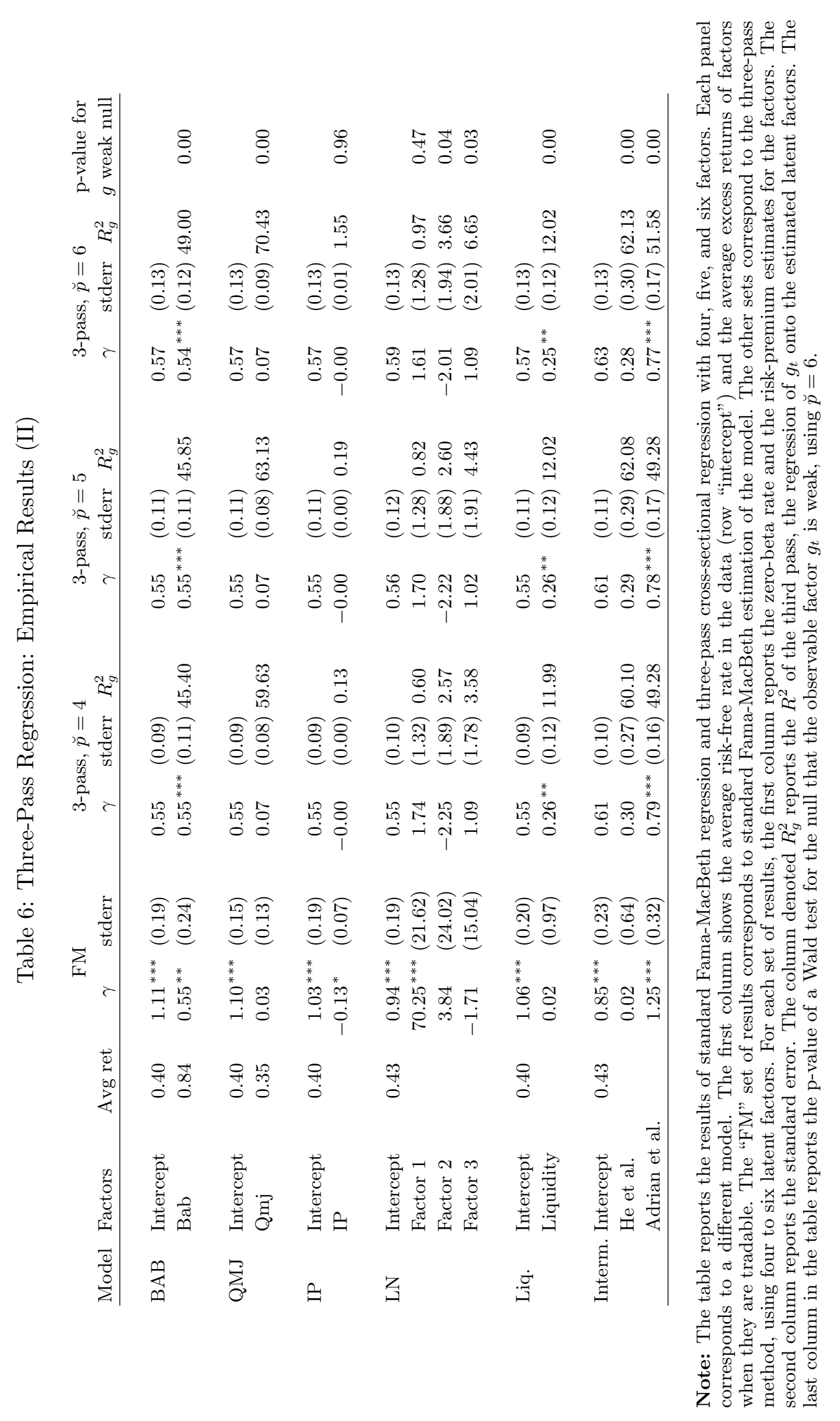


Table 7: Loading of Observable Factors onto Latent Factors (\% of Variation Explained)

\begin{tabular}{|c|c|c|c|c|c|c|c|}
\hline Model & Factors & Factor 1 & Factor 2 & Factor 3 & Factor 4 & Factor 5 & Factor 6 \\
\hline CAPM & RmRf & 91.0 & 6.3 & 1.7 & 0.1 & 0.8 & 0.2 \\
\hline \multirow[t]{3}{*}{ FF3 } & RmRf & 91.0 & 6.3 & 1.7 & 0.1 & 0.8 & 0.2 \\
\hline & SMB & 31.0 & 64.0 & 0.6 & 0.9 & 1.0 & 2.4 \\
\hline & HML & 7.0 & 1.3 & 75.5 & 4.9 & 1.4 & 9.9 \\
\hline \multirow[t]{4}{*}{ FF4 } & RmRf & 91.0 & 6.3 & 1.7 & 0.1 & 0.8 & 0.2 \\
\hline & SMB & 31.0 & 64.0 & 0.6 & 0.9 & 1.0 & 2.4 \\
\hline & HML & 7.0 & 1.3 & 75.5 & 4.9 & 1.4 & 9.9 \\
\hline & Mom & 3.1 & 0.3 & 2.0 & 93.5 & 0.4 & 0.7 \\
\hline \multirow[t]{5}{*}{ FF5 } & RmRf & 91.0 & 6.3 & 1.7 & 0.1 & 0.8 & 0.2 \\
\hline & SMB & 31.0 & 64.0 & 0.6 & 0.9 & 1.0 & 2.4 \\
\hline & HML & 7.0 & 1.3 & 75.5 & 4.9 & 1.4 & 9.9 \\
\hline & RMW & 17.2 & 37.4 & 15.4 & 4.1 & 7.6 & 18.3 \\
\hline & CMA & 19.8 & 0.0 & 60.6 & 0.1 & 2.0 & 17.5 \\
\hline \multirow[t]{4}{*}{$\mathrm{HXZ}$} & Mkt & 91.7 & 5.7 & 1.8 & 0.1 & 0.3 & 0.4 \\
\hline & $\mathrm{ME}$ & 28.4 & 60.8 & 5.7 & 1.3 & 1.3 & 2.5 \\
\hline & IA & 23.1 & 1.6 & 61.2 & 0.0 & 1.0 & 13.0 \\
\hline & $\mathrm{ROE}$ & 16.2 & 27.0 & 0.7 & 47.5 & 1.4 & 7.1 \\
\hline $\mathrm{BAB}$ & $\mathrm{Bab}$ & 0.9 & 3.6 & 72.7 & 15.4 & 0.9 & 6.4 \\
\hline QMJ & Qmj & 57.3 & 15.9 & 2.4 & 9.0 & 5.0 & 10.4 \\
\hline IP & IP Growth & 2.2 & 0.5 & 4.4 & 1.0 & 3.8 & 88.0 \\
\hline \multirow[t]{3}{*}{ LN } & Factor 1 & 21.3 & 10.1 & 27.3 & 2.8 & 22.6 & 15.9 \\
\hline & Factor 2 & 59.1 & 7.1 & 0.0 & 4.1 & 0.7 & 29.1 \\
\hline & Factor 3 & 19.9 & 0.2 & 21.0 & 12.8 & 12.8 & 33.3 \\
\hline Liq. & Liquidity & 95.0 & 2.8 & 1.8 & 0.2 & 0.2 & 0.0 \\
\hline \multirow[t]{2}{*}{ Interm. } & He et al. & 81.2 & 12.5 & 0.1 & 2.9 & 3.2 & 0.1 \\
\hline & Adrian et al. & 20.3 & 6.8 & 52.0 & 16.4 & 0.0 & 4.5 \\
\hline
\end{tabular}

Note: The table reports the decomposition of the variance of the observable factors $g_{t}$ explained by the six latent factors. Each row adds up to $100 \%$. 
Table 8: Risk Premia across Asset Classes

\begin{tabular}{|c|c|c|c|c|c|c|c|c|c|c|c|}
\hline \multirow[b]{2}{*}{ Model } & \multirow[b]{2}{*}{ Factors } & \multirow[b]{2}{*}{ Avg ret } & \multicolumn{3}{|c|}{202 equity } & \multicolumn{3}{|c|}{ FF25 + 100 non-equity } & \multicolumn{3}{|c|}{100 non-equity } \\
\hline & & & $\gamma$ & stderr & $R_{g}^{2}$ & $\gamma$ & stderr & $R_{g}^{2}$ & $\gamma$ & stderr & $R_{g}^{2}$ \\
\hline CAPM & RmRf & 0.47 & 0.29 & $(0.25)$ & 99.17 & $0.73^{* * *}$ & $(0.23)$ & 89.84 & $0.65^{* * *}$ & $(0.22)$ & 52.34 \\
\hline \multirow[t]{3}{*}{ FF3 } & RmRf & 0.47 & 0.29 & $(0.25)$ & 99.17 & $0.73^{* * *}$ & $(0.23)$ & 89.84 & $0.65^{* * *}$ & $(0.22)$ & 52.34 \\
\hline & SMB & 0.18 & 0.18 & $(0.14)$ & 97.17 & $0.22^{*}$ & $(0.12)$ & 31.13 & $0.13^{* *}$ & $(0.06)$ & 2.02 \\
\hline & HML & 0.41 & $0.25^{*}$ & $(0.13)$ & 76.93 & $0.16^{* *}$ & $(0.07)$ & 10.43 & 0.08 & $(0.07)$ & 4.43 \\
\hline \multirow[t]{4}{*}{ FF4 } & RmRf & 0.47 & 0.29 & $(0.25)$ & 99.17 & $0.73^{* * *}$ & $(0.23)$ & 89.84 & $0.65^{* * *}$ & $(0.22)$ & 52.34 \\
\hline & SMB & 0.18 & 0.18 & $(0.14)$ & 97.17 & $0.22^{*}$ & $(0.12)$ & 31.13 & $0.13^{* *}$ & $(0.06)$ & 2.02 \\
\hline & HML & 0.41 & $0.25^{*}$ & $(0.13)$ & 76.93 & $0.16^{* *}$ & $(0.07)$ & 10.43 & 0.08 & $(0.07)$ & 4.43 \\
\hline & Mom & 0.67 & $0.72^{* * *}$ & $(0.21)$ & 92.16 & -0.30 & $(0.18)$ & 6.49 & -0.28 & $(0.19)$ & 6.43 \\
\hline \multirow[t]{5}{*}{ FF5 } & RmRf & 0.47 & 0.29 & $(0.25)$ & 99.17 & $0.73^{* * *}$ & $(0.23)$ & 89.84 & $0.65^{* * *}$ & $(0.22)$ & 52.34 \\
\hline & SMB & 0.18 & 0.18 & $(0.14)$ & 97.17 & $0.22^{*}$ & $(0.12)$ & 31.13 & $0.13^{* *}$ & $(0.06)$ & 2.02 \\
\hline & HML & 0.41 & $0.25^{*}$ & $(0.13)$ & 76.93 & $0.16^{* *}$ & $(0.07)$ & 10.43 & 0.08 & $(0.07)$ & 4.43 \\
\hline & RMW & 0.29 & $0.16^{*}$ & $(0.09)$ & 52.13 & $-0.17^{* * *}$ & $(0.05)$ & 11.27 & $-0.17^{* * *}$ & $(0.05)$ & 6.92 \\
\hline & CMA & 0.40 & $0.17^{*}$ & $(0.09)$ & 53.85 & -0.04 & $(0.06)$ & 14.17 & -0.08 & $(0.06)$ & 9.09 \\
\hline \multirow[t]{4}{*}{ HXZ } & Mkt & 0.46 & 0.29 & $(0.25)$ & 99.08 & $0.76^{* * *}$ & $(0.24)$ & 90.07 & $0.69^{* * *}$ & $(0.22)$ & 52.71 \\
\hline & $\mathrm{ME}$ & 0.29 & $0.28^{*}$ & $(0.15)$ & 94.46 & $0.29^{* *}$ & $(0.12)$ & 29.92 & $0.19^{* * *}$ & $(0.07)$ & 2.56 \\
\hline & IA & 0.47 & $0.16^{*}$ & $(0.08)$ & 52.70 & -0.02 & $(0.06)$ & 13.64 & -0.05 & $(0.06)$ & 7.09 \\
\hline & $\mathrm{ROE}$ & 0.56 & $0.29^{* * *}$ & $(0.10)$ & 58.23 & $-0.22^{* *}$ & $(0.09)$ & 12.91 & $-0.20^{* *}$ & $(0.08)$ & 7.83 \\
\hline $\mathrm{BAB}$ & $\mathrm{Bab}$ & 0.87 & $0.59^{* * *}$ & $(0.14)$ & 50.20 & $0.22^{* *}$ & $(0.09)$ & 7.05 & 0.12 & $(0.11)$ & 8.91 \\
\hline QMJ & Qmj & 0.38 & 0.09 & $(0.11)$ & 72.74 & $-0.44^{* * *}$ & $(0.11)$ & 45.15 & $-0.40^{* * *}$ & $(0.10)$ & 26.95 \\
\hline IP & IP & & -0.00 & $(0.01)$ & 1.84 & 0.01 & $(0.01)$ & 0.79 & 0.01 & $(0.01)$ & 0.75 \\
\hline \multirow[t]{3}{*}{$\mathrm{LN}$} & Factor 1 & & 1.49 & $(1.31)$ & 0.88 & -1.13 & $(2.76)$ & 1.33 & -1.70 & $(2.79)$ & 1.21 \\
\hline & Factor 2 & & -1.85 & (1.96) & 3.44 & $-7.89^{* *}$ & $(3.86)$ & 4.40 & $-7.72^{* *}$ & $(3.89)$ & 4.28 \\
\hline & Factor 3 & & 0.24 & $(2.09)$ & 7.15 & -2.56 & $(3.83)$ & 3.59 & -3.55 & $(3.66)$ & 2.52 \\
\hline Liq. & Liquidity & & 0.22 & $(0.14)$ & 11.76 & 0.24 & $(0.18)$ & 11.45 & 0.19 & $(0.17)$ & 5.86 \\
\hline Interm. & He et al. & & 0.28 & $(0.30)$ & 62.13 & $1.03^{* * *}$ & $(0.30)$ & 52.14 & $0.95^{* * *}$ & $(0.30)$ & 34.87 \\
\hline & Adrian et al. & & $0.77^{* * *}$ & $(0.17)$ & 51.58 & $0.50^{* * *}$ & $(0.11)$ & 14.91 & $0.35^{* * *}$ & $(0.09)$ & 6.90 \\
\hline
\end{tabular}

Note: The table reports the results of risk premia estimation for various models using three-pass cross-sectional regression. The left side of the panel uses 202 equity portfolios as test assets. The center panel uses the 25 Fama-French portfolios plus 100 non-equity assets. The right panel uses only the 100 non-equity assets. Sample period covers 19702012. The number of factors $\breve{p}$ used is 6 for the left panel (as in our main analysis) and 5 for the middle and right panels. 
Table 9: Alternative Penalty for Latent Factor Estimation

\begin{tabular}{|c|c|c|c|c|c|c|c|}
\hline \multirow[b]{2}{*}{ Model } & \multirow[b]{2}{*}{ Factors } & \multicolumn{3}{|c|}{$\operatorname{PCA}(w=0)$} & \multicolumn{3}{|c|}{$\mathrm{CK}(w=1)$} \\
\hline & & $\breve{p}=4$ & $\breve{p}=5$ & $\breve{p}=6$ & $\breve{p}=4$ & $\breve{p}=5$ & $\breve{p}=6$ \\
\hline CAPM & RmRf & 0.51 & 0.52 & 0.52 & 0.51 & 0.52 & 0.52 \\
\hline \multirow[t]{3}{*}{ FF3 } & RmRf & 0.51 & 0.52 & 0.52 & 0.51 & 0.52 & 0.52 \\
\hline & SMB & 0.24 & 0.23 & 0.23 & 0.24 & 0.24 & 0.23 \\
\hline & HML & 0.21 & 0.21 & 0.22 & 0.22 & 0.22 & 0.22 \\
\hline \multirow[t]{4}{*}{$\mathrm{FF} 4$} & RmRf & 0.51 & 0.52 & 0.52 & 0.51 & 0.52 & 0.52 \\
\hline & SMB & 0.24 & 0.23 & 0.23 & 0.24 & 0.24 & 0.23 \\
\hline & HML & 0.21 & 0.21 & 0.22 & 0.22 & 0.22 & 0.22 \\
\hline & Mom & 0.77 & 0.76 & 0.76 & 0.81 & 0.80 & 0.81 \\
\hline \multirow[t]{5}{*}{ FF5 } & RmRf & 0.51 & 0.52 & 0.52 & 0.51 & 0.52 & 0.52 \\
\hline & SMB & 0.24 & 0.23 & 0.23 & 0.24 & 0.24 & 0.23 \\
\hline & HML & 0.21 & 0.21 & 0.22 & 0.22 & 0.22 & 0.22 \\
\hline & RMW & 0.15 & 0.15 & 0.15 & 0.15 & 0.16 & 0.15 \\
\hline & CMA & 0.13 & 0.13 & 0.13 & 0.14 & 0.14 & 0.14 \\
\hline \multirow[t]{4}{*}{ HXZ } & Mkt & 0.50 & 0.51 & 0.51 & 0.50 & 0.51 & 0.51 \\
\hline & $\mathrm{ME}$ & 0.33 & 0.32 & 0.31 & 0.33 & 0.32 & 0.31 \\
\hline & IA & 0.13 & 0.13 & 0.14 & 0.14 & 0.14 & 0.15 \\
\hline & $\mathrm{ROE}$ & 0.30 & 0.31 & 0.30 & 0.32 & 0.32 & 0.31 \\
\hline $\mathrm{BAB}$ & $\mathrm{Bab}$ & 0.59 & 0.60 & 0.60 & 0.61 & 0.62 & 0.62 \\
\hline QMJ & Qmj & 0.05 & 0.06 & 0.05 & 0.06 & 0.07 & 0.06 \\
\hline IP & IP & -0.00 & -0.00 & -0.00 & -0.00 & -0.00 & -0.00 \\
\hline \multirow[t]{3}{*}{$\mathrm{LN}$} & Factor 1 & 1.86 & 1.91 & 1.90 & 1.91 & 1.96 & 1.96 \\
\hline & Factor 2 & -2.78 & -2.80 & -2.80 & -2.86 & -2.88 & -2.88 \\
\hline & Factor 3 & 2.00 & 2.11 & 2.12 & 1.99 & 2.10 & 2.10 \\
\hline Liq. & Liquidity & 0.33 & 0.33 & 0.33 & 0.34 & 0.34 & 0.34 \\
\hline \multirow[t]{2}{*}{ Interm. } & He et al. & 0.50 & 0.51 & 0.51 & 0.49 & 0.51 & 0.51 \\
\hline & Adrian et al. & 0.89 & 0.89 & 0.90 & 0.92 & 0.92 & 0.93 \\
\hline
\end{tabular}

Note: The table reports the results of risk premia estimation for various models using three-pass cross-sectional regression, using different penalty functions to extract the latent factors. We report results for $\breve{p}=4,5,6$. The left panel uses standard PCA as in our baseline case. The right panel extracts factors using the Connor and Korajczyk (1986, 1988) approach, which gives weight to the cross-sectional $R^{2}$. Following Connor and Korajczyk (1986, 1988), the zero-beta rate is imposed to be equal to the risk-free rate, which is the reason for the difference between our baseline results of Tables 5 and 6 and the left panel in this table. Sample period covers 1970-2012. 


\section{Appendix A Mathematical Proofs}

\section{Appendix A.1 Proofs of Main Theorems}

Proof of Proposition 1. Without loss of generality, we assume that $\eta$ is a $1 \times p$ vector. Consider an invertible matrix $H$ such that its first row is equal to $\eta$. The model (1) can then be written equivalently as:

$$
\begin{aligned}
r_{t} & =\iota_{n} \gamma_{0}+\alpha+\beta H^{-1} H \gamma+\beta H^{-1} H v_{t}+u_{t} \\
& :=\iota_{n} \gamma_{0}+\alpha+\tilde{\beta} \tilde{\gamma}+\tilde{\beta} \tilde{v}_{t}+u_{t},
\end{aligned}
$$

where $\tilde{\beta}=\beta H^{-1}, \tilde{\gamma}=H \gamma$, and $\tilde{v}_{t}=H v_{t}$. The expected excess return of a portfolio with no idiosyncratic risk or alpha, which has unit beta on the $i$ th factor in $\tilde{v}_{t}$ and zero beta on the rest factors is $\operatorname{E}\left(\gamma_{0}+\right.$ $\left.e_{i}^{\top} \tilde{\gamma}+e_{i}^{\top} \tilde{v}_{t}\right)-\gamma_{0}=\tilde{\gamma}_{i}$. By definition, the risk premium of $\tilde{v}_{t}$ is $\tilde{\gamma}$. Since $\tilde{g}_{t}=e_{1}^{\top} \tilde{v}_{t}$, its risk premium is $e_{1}^{\top} \tilde{\gamma}=e_{1}^{\top} H \gamma=\eta \gamma$.

To establish the second statement, suppose $\left(\tilde{v}_{1}: \tilde{v}_{2}: \ldots: \tilde{v}_{T}\right)$ is the new set of factors. Because it shares the same row space as $\left(v_{1}: v_{2}: \ldots: v_{T}\right)$, there exists an invertible matrix $H$, such that $\tilde{v}_{t}=H v_{t}$, for all $t=1,2, \ldots, T$. By the above result, the risk premium of $\tilde{v}_{t}, \tilde{\gamma}$, is therefore $H \gamma$. Since $g_{t}=\eta v_{t}$, it follows that $g_{t}=\eta H^{-1} \tilde{v}_{t}$. Applying the above result again, we have the risk premium of $g_{t}$ is equal to $\eta H^{-1} H \gamma=\eta \gamma$.

Proof of Theorem 1. We take two steps to prove it.

Step 1: Since

$$
\bar{R}^{\top} \bar{R}-\bar{V}^{\top} \beta^{\top} \beta \bar{V}=\bar{U}^{\top} \beta \bar{V}+\bar{V}^{\top} \beta^{\top} \bar{U}+\bar{U}^{\top} \bar{U}
$$

then by Weyl's inequality, we have, for $1 \leq j \leq p$,

$$
\left|\lambda_{j}\left(\bar{R}^{\top} \bar{R}\right)-\lambda_{j}\left(\bar{V}^{\top} \beta^{\top} \beta \bar{V}\right)\right| \leq\left\|\bar{U}^{\top} \bar{U}\right\|+\left\|\bar{U}^{\top} \beta \bar{V}\right\|+\left\|\bar{V}^{\top} \beta^{\top} \bar{U}\right\| .
$$

We analyze the terms on the right-hand side one by one.

(i) To begin with, write $\Gamma^{u}=\left(\gamma_{n, t t^{\prime}}\right)$. Note that

$$
\left\|\bar{U}^{\top} \bar{U}-n \Gamma^{u}\right\| \leq\left\|U^{\top} U-n \Gamma^{u}\right\|_{\mathrm{F}}+2\left\|\iota_{T} \bar{u}^{\top} U\right\|_{\mathrm{F}}+\left\|\iota_{T} \bar{u}^{\top} \bar{u} \iota_{T}^{\top}\right\|_{\mathrm{F}} .
$$

By Assumption 3(iv),

$$
\mathrm{E}\left\|U^{\top} U-n \Gamma^{u}\right\|_{\mathrm{F}}^{2}=\sum_{s=1}^{T} \sum_{t=1}^{T} \mathrm{E}\left(\sum_{j=1}^{n}\left(u_{j s} u_{j t}-\mathrm{E}\left(u_{j s} u_{j t}\right)\right)\right)^{2} \leq K n T^{2},
$$


and by Assumption 3(i),

$$
\begin{aligned}
& \mathrm{E}\|\bar{u}\|_{\mathrm{F}}^{2}=T^{-2} \mathrm{E} \sum_{i=1}^{n} \sum_{t=1}^{T} \sum_{t^{\prime}=1}^{T} u_{i t} u_{i t^{\prime}} \leq n T^{-2} \sum_{t=1}^{T} \sum_{t^{\prime}=1}^{T}\left|\gamma_{n, t t^{\prime}}\right| \leq K n T^{-1}, \\
& \mathrm{E}\|U\|_{\mathrm{F}}^{2}=\sum_{i=1}^{n} \sum_{t=1}^{T} \mathrm{E} u_{i t}^{2} \leq n \sum_{t=1}^{T} \gamma_{n, t t} \leq K n T,
\end{aligned}
$$

it follows that

$$
\left\|\iota_{T} \bar{u}^{\top} U\right\|_{\mathrm{F}} \leq\left\|\iota_{T}\right\|_{\mathrm{F}}\left\|\bar{u}^{\top}\right\|_{\mathrm{F}}\|U\|_{\mathrm{F}}=O_{p}\left(n T^{1 / 2}\right), \quad\left\|\iota_{T} \bar{u}^{\top} \bar{u} \iota_{T}^{\top}\right\|_{\mathrm{F}} \leq\left\|\iota_{T}\right\|_{\mathrm{F}}^{2}\left\|\bar{u}^{\top}\right\|_{\mathrm{F}}^{2}=O_{p}(n),
$$

and hence that

$$
\left\|\bar{U}^{\top} \bar{U}-n \Gamma^{u}\right\|=O_{p}\left(n^{1 / 2} T\right)+O_{p}\left(n T^{1 / 2}\right) .
$$

Next, writing $\rho_{n, s t}=\gamma_{n, s t} / \sqrt{\gamma_{n, s s} \gamma_{n, t}}$, by Assumption 3(i) and the fact that $\left|\rho_{n, s t}\right| \leq 1$,

$$
\begin{aligned}
\left\|\Gamma^{u}\right\|_{\mathrm{F}}^{2} & =\sum_{s=1}^{T} \sum_{t=1}^{T} \gamma_{n, s t}^{2}=\sum_{s=1}^{T} \sum_{t=1}^{T} \gamma_{n, s s} \gamma_{n, t t} \rho_{n, s t}^{2} \\
& \leq K \sum_{s=1}^{T} \sum_{t=1}^{T}\left|\gamma_{n, s s} \gamma_{n, t t}\right|^{1 / 2}\left|\rho_{n, s t}\right| \leq K \sum_{s=1}^{T} \sum_{t=1}^{T}\left|\gamma_{n, s t}\right| \leq K T,
\end{aligned}
$$

so we have $n\left\|\Gamma^{u}\right\|=O_{p}\left(n T^{1 / 2}\right)$.

Therefore, we obtain

$$
\left\|\bar{U}^{\top} \bar{U}\right\| \leq\left\|\bar{U}^{\top} \bar{U}-n \Gamma^{u}\right\|+n\left\|\Gamma^{u}\right\|=O_{p}\left(n T^{1 / 2}\right)+O_{p}\left(n^{1 / 2} T\right)
$$

(ii) By Assumption 5, we have $\|\beta\|_{\text {MAX }} \leq K$. By Assumption 3(ii)(iii), we have

$$
\begin{aligned}
& \mathrm{E}\left\|U^{\top} \beta\right\|_{\mathrm{F}}^{2}=\mathrm{E} \sum_{j=1}^{p} \sum_{t=1}^{T}\left(\sum_{i=1}^{n} \beta_{i j} u_{i t}\right)^{2} \leq K \sum_{j=1}^{p} \sum_{t=1}^{T} \sum_{i=1}^{n} \sum_{i^{\prime}=1}^{n}\left|\sigma_{i i^{\prime}, t}\right| \leq K n T, \\
& \mathrm{E}\left\|\bar{u}^{\top} \beta\right\|_{\mathrm{F}}^{2} \leq \mathrm{E} \sum_{k=1}^{p}\left(\sum_{i=1}^{n} \bar{u}_{i} \beta_{i k}\right)^{2} \leq K T^{-2} \sum_{k=1}^{p} \sum_{i=1}^{n} \sum_{i^{\prime}=1}^{n} \sum_{t=1}^{T} \sum_{t^{\prime}=1}^{T}\left|\sigma_{i i^{\prime}, t t^{\prime}}\right| \leq K n T^{-1},
\end{aligned}
$$

it follows that

$$
\left\|\bar{U}^{\top} \beta\right\|_{\mathrm{F}} \leq\left\|U^{\top} \beta\right\|_{\mathrm{F}}+\left\|\iota_{T}\right\|_{\mathrm{F}}\left\|\bar{u}^{\top} \beta\right\|_{\mathrm{F}}=O_{p}\left(n^{1 / 2} T^{1 / 2}\right) .
$$

Also, by Assumption 4,

$$
T^{-1}\left\|\bar{V} \bar{V}^{\top}\right\|_{\mathrm{MAX}} \leq\left\|T^{-1} V V^{\top}-\Sigma^{v}\right\|_{\mathrm{MAX}}+\left\|\Sigma^{v}\right\|_{\mathrm{MAX}}+\left\|\bar{v} \bar{v}^{\top}\right\|_{\mathrm{MAX}} \leq K
$$


we have

$$
\|\bar{V}\| \leq\left\|\bar{V} \bar{V}^{\top}\right\|^{1 / 2} \leq K\left\|\bar{V} \bar{V}^{\top}\right\|_{\mathrm{MAX}}^{1 / 2}=O_{p}\left(T^{1 / 2}\right)
$$

Therefore, we have

$$
\left\|\bar{V}^{\top} \beta^{\top} \bar{U}\right\|=\left\|\bar{U}^{\top} \beta \bar{V}\right\| \leq\left\|\bar{U}^{\top} \beta\right\|_{\mathrm{F}}\|\bar{V}\|=O_{p}\left(n^{1 / 2} T\right) .
$$

Combining (i) and (ii), we have for $1 \leq j \leq p$,

$$
n^{-1} T^{-1}\left|\lambda_{j}\left(\bar{R}^{\top} \bar{R}\right)-\lambda_{j}\left(\bar{V}^{\top} \beta^{\top} \beta \bar{V}\right)\right|=O_{p}\left(n^{-1 / 2}+T^{-1 / 2}\right)=o_{p}(1) .
$$

(iii) Moreover, by Assumption 5, (A.11), and Weyl's inequality again,

$$
\left|n^{-1} T^{-1} \lambda_{j}\left(\bar{V}^{\top} \beta^{\top} \beta \bar{V}\right)-T^{-1} \lambda_{j}\left(\bar{V}^{\top} \Sigma^{\beta} \bar{V}\right)\right| \leq\left\|n^{-1} \beta^{\top} \beta-\Sigma^{\beta}\right\| T^{-1}\left\|\bar{V}^{\top}\right\|\|\bar{V}\|=o_{p}(1),
$$

and combined with Assumption 4, and the fact that $\|\bar{v}\| \leq K\|\bar{v}\|_{\text {MAX }}=O_{p}\left(T^{-1 / 2}\right)$,

$$
\begin{aligned}
& \left|T^{-1} \lambda_{j}\left(\bar{V}^{\top} \Sigma^{\beta} \bar{V}\right)-\lambda_{j}\left(\left(\Sigma^{\beta}\right)^{1 / 2} \Sigma^{v}\left(\Sigma^{\beta}\right)^{1 / 2}\right)\right| \\
\leq & \left\|T^{-1} \bar{V} \bar{V}^{\top}-\Sigma^{v}\right\|\left\|\Sigma^{\beta}\right\| \leq\left(\left\|T^{-1} V V^{\top}-\Sigma^{v}\right\|+\left\|\bar{v} \bar{v}^{\top}\right\|\right)\left\|\Sigma^{\beta}\right\|=o_{p}(1),
\end{aligned}
$$

where we also use the fact that the non-zero eigenvalues of $\bar{V}^{\top} \Sigma^{\beta} \bar{V}$ are identical to the non-zero eigenvalues of $\left(\Sigma^{\beta}\right)^{1 / 2} \bar{V} \bar{V}^{\top}\left(\Sigma^{\beta}\right)^{1 / 2}$. Therefore, for $1 \leq j \leq p$,

$$
\left|n^{-1} T^{-1} \lambda_{j}\left(\bar{R}^{\top} \bar{R}\right)-\lambda_{j}\left(\left(\Sigma^{\beta}\right)^{1 / 2} \Sigma^{v}\left(\Sigma^{\beta}\right)^{1 / 2}\right)\right|=o_{p}(1)
$$

Step 2: By Assumptions 4 and 5, there exists $0<K_{1}, K_{2}<\infty$, such that

$$
K_{1}<\lambda_{\min }\left(\Sigma^{v}\right) \lambda_{\min }\left(\Sigma^{\beta}\right) \leq \lambda_{\min }\left(\Sigma^{v} \Sigma^{\beta}\right) \leq \lambda_{\max }\left(\Sigma^{v} \Sigma^{\beta}\right) \leq \lambda_{\max }\left(\Sigma^{v}\right) \lambda_{\max }\left(\Sigma^{\beta}\right)<K_{2}
$$

Therefore the eigenvalues of $\left(\Sigma^{\beta}\right)^{1 / 2} \Sigma^{v}\left(\Sigma^{\beta}\right)^{1 / 2}$ are bounded away from 0 and $\infty$, we have by (A.13), for $1 \leq j \leq p$,

$$
K_{1}<n^{-1} T^{-1} \lambda_{j}\left(\bar{R}^{\top} \bar{R}\right)<K_{2}
$$

On the other hand, we can write

$$
\bar{R} \bar{R}^{\top}=\tilde{\beta} \bar{V} \bar{V}^{\top} \tilde{\beta}^{\top}+\bar{U}\left(\mathbb{I}_{T}-\bar{V}^{\top}\left(\bar{V} \bar{V}^{\top}\right)^{-1} \bar{V}\right) \bar{U}^{\top}
$$

where $\tilde{\beta}=\beta+U \bar{V}^{\top}\left(\bar{V} \bar{V}^{\top}\right)^{-1}$. By (4.3.2a) of Theorem 4.3.1 and (4.3.14) of Corollary 4.3.12 in Horn and 
Johnson (2013), for $p+1 \leq j \leq n$, we have

$$
\lambda_{j}\left(\bar{R} \bar{R}^{\top}\right) \leq \lambda_{j-p}\left(\bar{U}\left(\mathbb{I}_{T}-\bar{V}^{\top}\left(\bar{V} \bar{V}^{\top}\right)^{-1} \bar{V}\right) \bar{U}^{\top}\right)+\lambda_{p+1}\left(\tilde{\beta} \bar{V} \bar{V}^{\top} \tilde{\beta}\right) \leq \lambda_{j-p}\left(\bar{U} \bar{U}^{\top}\right) \leq \lambda_{1}\left(\bar{U} \bar{U}^{\top}\right)
$$

Moreover, by (A.6), we have

$$
\lambda_{1}\left(\bar{U} \bar{U}^{\top}\right)=\left\|\bar{U}^{\top} \bar{U}\right\|=O_{p}\left(n T^{1 / 2}\right)+O_{p}\left(n^{1 / 2} T\right),
$$

hence for $p+1 \leq j \leq n$, there exists some $K>0$, such that

$$
n^{-1} T^{-1} \lambda_{j}\left(\bar{R}^{\top} \bar{R}\right) \leq K\left(n^{-1 / 2}+T^{-1 / 2}\right)
$$

Now we define, for $1 \leq j \leq n$,

$$
f(j)=n^{-1} T^{-1} \lambda_{j}\left(\bar{R}^{\top} \bar{R}\right)+j \times \phi(n, T) .
$$

(A.14) and (A.16) together imply that for $1 \leq j \leq p$,

$$
\begin{aligned}
f(j)-f(p+1) & =n^{-1} T^{-1}\left(\lambda_{j}\left(\bar{R}^{\top} \bar{R}\right)-\lambda_{p+1}\left(\bar{R}^{\top} \bar{R}\right)\right)+(j-p-1) \phi(n, T) \\
& >\lambda_{j}\left(\left(\Sigma^{\beta}\right)^{1 / 2} \Sigma^{v}\left(\Sigma^{\beta}\right)^{1 / 2}\right)+o_{p}(1)>K
\end{aligned}
$$

for some $K>0$; and for $p+1<j \leq n$, we have

$$
\mathrm{P}(f(j)<f(p+1))=\mathrm{P}\left((j-p-1) \phi(n, T)<n^{-1} T^{-1}\left(\lambda_{p+1}\left(\bar{R}^{\top} \bar{R}\right)-\lambda_{j}\left(\bar{R}^{\top} \bar{R}\right)\right)\right) \rightarrow 0 .
$$

Therefore, $p+1=\arg \min _{1 \leq j \leq p} f(j)$ holds with probability approaching 1 , and hence $\widehat{p} \stackrel{p}{\longrightarrow} p$.

Proof of Theorem 2. Let $\widehat{\Lambda}$ be the $p \times p$ diagonal matrix of the $p$ largest eigenvalues of $n^{-1} T^{-1} \bar{R}^{\top} \bar{R}$. We define a $p \times p$ matrix:

$$
H=n^{-1} T^{-1} \widehat{\Lambda}^{-1} \widehat{V} \bar{V}^{\top} \beta^{\top} \beta .
$$

We use the following decomposition:

$$
\begin{aligned}
& \widetilde{\Gamma}-\left(\begin{array}{c}
\gamma_{0} \\
H \gamma
\end{array}\right) \\
& =\left(\left(\iota_{n}: \widehat{\beta}\right)^{\top}\left(\iota_{n}: \widehat{\beta}\right)\right)^{-1}\left(\iota_{n}: \widehat{\beta}\right)^{\top}((\beta-\widehat{\beta} H) \gamma+\beta \bar{v}+\alpha+\bar{u}) \\
& =\left(\begin{array}{c}
0 \\
H \bar{v}
\end{array}\right)+\left\{\frac{1}{n}\left(\begin{array}{cc}
\iota_{n}^{\top} \iota_{n} & \iota_{n}^{\top} \widehat{\beta} \\
\widehat{\beta}^{\top} \iota_{n} & \widehat{\beta}^{\top} \widehat{\beta}
\end{array}\right)\right\}^{-1}\left\{\frac{1}{n}\left(\begin{array}{c}
\iota_{n}^{\top} \alpha \\
H^{-\top} \beta^{\top} \alpha
\end{array}\right)+\frac{1}{n}\left(\begin{array}{c}
\iota_{n}^{\top} \bar{u}+\iota_{n}^{\top}(\beta-\widehat{\beta} H) \gamma \\
H^{-\top} \beta^{\top} \bar{u}+H^{-\top} \beta^{\top}(\beta-\widehat{\beta} H) \gamma
\end{array}\right)\right. \\
& \left.+\frac{1}{n}\left(\begin{array}{c}
\iota_{n}^{\top}(\beta-\widehat{\beta} H) \bar{v} \\
\left(\widehat{\beta}-\beta H^{-1}\right)^{\top}(\alpha+\bar{u})+H^{-\top} \beta^{\top}(\beta-\widehat{\beta} H) \bar{v}+\left(\widehat{\beta}^{\top}-H^{-\top} \beta^{\top}\right)(\beta-\widehat{\beta} H)(\gamma+\bar{v})
\end{array}\right)\right\} .
\end{aligned}
$$


Note that

$$
\widehat{\eta}-\eta H^{-1}=\eta H^{-1}(H \bar{V}-\widehat{V}) \widehat{V}^{\top}\left(\widehat{V} \widehat{V}^{\top}\right)^{-1}+\bar{Z} \widehat{V}^{\top}\left(\widehat{V} \widehat{V}^{\top}\right)^{-1}
$$

and by Lemma 7(a) and (b), we have

$$
\widehat{\eta}-\eta H^{-1}=T^{-1} \bar{Z} \bar{V}^{\top} H^{\top}+O_{p}\left(n^{-1}+T^{-1}\right) .
$$

Moreover, by Assumptions 4 and 6, as well as Lemma 2, we have

$$
\left\|T^{-1} \bar{Z} \bar{V}^{\top} H^{\top}\right\|_{\mathrm{MAX}} \leq\left(\left\|T^{-1} Z V^{\top}\right\|_{\mathrm{MAX}}+\left\|\bar{z} \bar{v}^{\top}\right\|_{\mathrm{MAX}}\right)\|H\|_{\mathrm{MAX}}=O_{p}\left(T^{-1 / 2}\right),
$$

it follows that

$$
\left\|\widehat{\eta}-\eta H^{-1}\right\|=O_{p}\left(n^{-1}+T^{-1 / 2}\right) .
$$

Using this, and by Lemmas 2, 4, 5, 6, 7, and 8, we have

$$
\begin{aligned}
\widehat{\Gamma}-\left(\begin{array}{c}
\gamma_{0} \\
\eta \gamma
\end{array}\right)= & \left.\begin{array}{c}
0 \\
T^{-1} \bar{Z} \bar{V}^{\top}\left(\Sigma^{v}\right)^{-1} \gamma+\eta \bar{v}
\end{array}\right) \\
& +\left(\begin{array}{cc}
1 & 0 \\
0 & \eta
\end{array}\right)\left\{\frac{1}{n}\left(\begin{array}{cc}
\iota_{n}^{\top} \iota_{n} & \iota_{n}^{\top} \beta \\
\beta^{\top} \iota_{n} & \beta^{\top} \beta
\end{array}\right)+o_{p}(1)\right\}^{-1} \times\left\{\frac{1}{n}\left(\begin{array}{c}
\iota_{n}^{\top} \alpha \\
\beta^{\top} \alpha
\end{array}\right)+o_{p}\left(n^{-1 / 2}+T^{-1 / 2}\right)\right\} .
\end{aligned}
$$

Moreover, by Cramér-Wold theorem and Lyapunov's central limit theorem, we can obtain

$$
n^{-1 / 2}\left(\begin{array}{c}
\iota_{n}^{\top} \alpha \\
\beta^{\top} \alpha
\end{array}\right) \stackrel{\mathcal{L}}{\longrightarrow} \mathcal{N}\left(\left(\begin{array}{c}
0 \\
0
\end{array}\right),\left(\begin{array}{cc}
1 & \beta_{0}^{\top} \\
\beta_{0} & \Sigma^{\beta}
\end{array}\right)\left(\sigma^{\alpha}\right)^{2}\right)
$$

where we use $\left\|n^{-1} \beta^{\top} \iota_{n}-\beta_{0}\right\|_{\text {MAX }}=o(1)$ and $\left\|n^{-1} \beta^{\top} \beta-\Sigma^{\beta}\right\|_{\text {MAX }}=o(1)$. Also, Assumptions 5 and 10 ensure that $\left(1-\beta_{0}^{\top}\left(\Sigma^{\beta}\right)^{-1} \beta_{0}\right)$ and $\left(\Sigma^{\beta}-\beta_{0} \beta_{0}^{\top}\right)$ are invertible. Therefore, by the Delta method, we have

$$
n^{1 / 2}\left(\widehat{\gamma}_{0}-\gamma_{0}\right) \stackrel{\mathcal{L}}{\longrightarrow} \mathcal{N}\left(0,\left(1-\beta_{0}^{\top}\left(\Sigma^{\beta}\right)^{-1} \beta_{0}\right)^{-1}\left(\sigma^{\alpha}\right)^{2}\right)
$$

Similarly, we have

$$
n^{1 / 2}\left(\begin{array}{cc}
0 & \eta
\end{array}\right)\left\{\frac{1}{n}\left(\begin{array}{cc}
\iota_{n}^{\top} \iota_{n} & \iota_{n}^{\top} \beta \\
\beta^{\top} \iota_{n} & \beta^{\top} \beta
\end{array}\right)+o_{p}(1)\right\}^{-1} \times \frac{1}{n}\left(\begin{array}{c}
\iota_{n}^{\top} \alpha \\
\beta^{\top} \alpha
\end{array}\right) \stackrel{\mathcal{L}}{\longrightarrow} \mathcal{N}(0, \Upsilon),
$$

where

$$
\Upsilon=\left(\sigma^{\alpha}\right)^{2} \eta\left(\Sigma^{\beta}-\beta_{0} \beta_{0}^{\top}\right)^{-1} \eta^{\top}
$$


On the other hand, since

$$
\begin{aligned}
\operatorname{vec}\left(T^{-1} \bar{Z} \bar{V}^{\top}\left(\Sigma^{v}\right)^{-1} \gamma\right) & =\left(\gamma^{\top}\left(\Sigma^{v}\right)^{-1} \otimes \mathbb{I}_{d}\right)\left(\operatorname{vec}\left(T^{-1} Z V^{\top}\right)+\operatorname{vec}\left(\bar{z} \bar{v}^{\top}\right)\right) \\
& =\left(\gamma^{\top}\left(\Sigma^{v}\right)^{-1} \otimes \mathbb{I}_{d}\right) \operatorname{vec}\left(T^{-1} Z V^{\top}\right)+O_{p}\left(T^{-1}\right)
\end{aligned}
$$

it follows from Assumption 11 that

$$
\begin{gathered}
T^{1 / 2}\left(\begin{array}{c}
T^{-1} \bar{Z} \bar{V}^{\top}\left(\Sigma^{v}\right)^{-1} \gamma \\
\eta \bar{v}
\end{array}\right) \\
\stackrel{\mathcal{L}}{\longrightarrow} \mathcal{N}\left(\left(\begin{array}{l}
0 \\
0
\end{array}\right),\left(\begin{array}{cc}
\left(\gamma^{\top}\left(\Sigma^{v}\right)^{-1} \otimes \mathbb{I}_{d}\right) \Pi_{11}\left(\left(\Sigma^{v}\right)^{-1} \gamma \otimes \mathbb{I}_{d}\right) & \left(\gamma^{\top}\left(\Sigma^{v}\right)^{-1} \otimes \mathbb{I}_{d}\right) \Pi_{12} \eta^{\top} \\
. & \eta \Pi_{22} \eta^{\top}
\end{array}\right) .\right.
\end{gathered}
$$

Therefore, by the Delta method, we obtain:

$$
T^{1 / 2}\left(T^{-1} \bar{Z} \bar{V}^{\top}\left(\Sigma^{v}\right)^{-1} \gamma+\eta \bar{v}\right) \stackrel{\mathcal{L}}{\longrightarrow} \mathcal{N}(0, \Phi)
$$

where

$$
\begin{aligned}
\Phi= & \left(\gamma^{\top}\left(\Sigma^{v}\right)^{-1} \otimes \mathbb{I}_{d}\right) \Pi_{11}\left(\left(\Sigma^{v}\right)^{-1} \gamma \otimes \mathbb{I}_{d}\right)+\left(\gamma^{\top}\left(\Sigma^{v}\right)^{-1} \otimes \mathbb{I}_{d}\right) \Pi_{12} \eta^{\top} \\
& +\eta \Pi_{21}\left(\left(\Sigma^{v}\right)^{-1} \gamma \otimes \mathbb{I}_{d}\right)+\eta \Pi_{22} \eta^{\top} .
\end{aligned}
$$

By the same asymptotic independence argument as in the proof of Theorem 3 in Bai (2003), we establish the desired result:

$$
\left(T^{-1} \Phi+n^{-1} \Upsilon\right)^{-1 / 2}(\widehat{\gamma}-\eta \gamma) \stackrel{\mathcal{L}}{\longrightarrow} \mathcal{N}\left(0, \mathbb{I}_{d}\right)
$$

Proof of Theorem 3. By Assumptions 4, 5, and 10, Lemma 4, (A.8), and (A.21), we have

$$
\begin{aligned}
& n^{-1} \iota_{n}^{\top} \bar{r}=\gamma_{0}+\beta_{0}^{\top} \gamma+O_{p}\left(n^{-1 / 2}+T^{-1 / 2}\right), \\
& n^{-1} \bar{r}^{\top} \bar{r}=\gamma^{\top} \Sigma^{\beta} \gamma+\gamma_{0}^{2}+\left(\sigma^{\alpha}\right)^{2}+\gamma^{\top} \beta_{0} \gamma_{0}+\beta_{0}^{\top} \gamma \gamma_{0}+O_{p}\left(n^{-1 / 2}+T^{-1 / 2}\right),
\end{aligned}
$$

it then follows that

$$
n^{-1} \bar{r}^{\top} \mathbb{M}_{\iota_{n}} \bar{r}=n^{-1} \bar{r}^{\top} \bar{r}-\left(n^{-1} \iota_{n}^{\top} \bar{r}\right)^{2}=\gamma^{\top}\left(\Sigma^{\beta}-\beta_{0} \beta_{0}^{\top}\right) \gamma+\left(\sigma^{\alpha}\right)^{2}+o_{p}(1)
$$

On the other hand, by Assumption 4, Lemma 3, (A.2), we have

$$
\begin{aligned}
n^{-1}\left\|H^{\top} \widehat{\beta}^{\top} \mathbb{M}_{\iota_{n}} \bar{r}-\beta^{\top} \mathbb{M}_{\iota_{n}} \bar{r}\right\|_{\mathrm{MAX}} & =\left\|\left(H^{\top} \widehat{\beta}^{\top}-\beta^{\top}\right) \mathbb{M}_{\iota_{n}}(\alpha+\beta \gamma+\beta \bar{v}+\bar{u})\right\|_{\mathrm{MAX}} \\
& \leq n^{-1}\left\|H^{\top} \widehat{\beta}^{\top}-\beta^{\top}\right\|_{\mathrm{F}}\|\alpha+\beta \gamma+\beta \bar{v}+\bar{u}\|_{\mathrm{F}}=O_{p}\left(n^{-1 / 2}+T^{-1 / 2}\right) .
\end{aligned}
$$


Similarly, we have

$$
\begin{aligned}
& n^{-1} \beta^{\top} \mathbb{M}_{\iota_{n}} \bar{r}=\left(\Sigma^{\beta}-\beta_{0} \beta_{0}^{\top}\right) \gamma+o_{p}(1), \\
& n^{-1} \beta^{\top} \mathbb{M}_{\iota_{n}} \beta=\Sigma^{\beta}-\beta_{0} \beta_{0}^{\top}+o_{p}(1),
\end{aligned}
$$

therefore, we obtain

$$
\left(n^{-1} \beta^{\top} \mathbb{M}_{\iota_{n}} \bar{r}\right)^{\top}\left(n^{-1} \beta^{\top} \mathbb{M}_{\iota_{n}} \beta\right)^{-1}\left(n^{-1} \beta^{\top} \mathbb{M}_{\iota_{n}} \bar{r}\right)=\gamma^{\top}\left(\Sigma^{\beta}-\beta_{0} \beta_{0}^{\top}\right) \gamma+o_{p}(1),
$$

which establishes $\widehat{\mathrm{R}}_{v}^{2} \stackrel{p}{\longrightarrow} \mathrm{R}_{v}^{2}$.

By Lemma 2, (A.42), (A.20) and the fact that $\|\eta\|_{\text {MAX }} \leq K$, we have

$$
\begin{aligned}
& \left\|T^{-1} \widehat{\eta} \widehat{V} \widehat{V}^{\top} \eta^{\top}-\eta \Sigma^{v} \eta^{\top}\right\|_{\mathrm{MAX}} \\
\leq & \left\|\left(\widehat{\eta}-\eta H^{-1}\right)\left(\widehat{\eta}-\eta H^{-1}\right)^{\top}\right\|_{\mathrm{MAX}}+\left\|\left(\widehat{\eta}-\eta H^{-1}\right) H^{-\top} \eta^{\top}\right\|_{\mathrm{MAX}}+\left\|\eta H^{-1}\left(\widehat{\eta}-\eta H^{-1}\right)^{\top}\right\|_{\mathrm{MAX}} \\
& +\left\|\eta\left(H^{-1} H^{-\top}-\Sigma^{v}\right) \eta^{\top}\right\|_{\mathrm{MAX}} \\
= & O_{p}\left(n^{-1 / 2}+T^{-1 / 2}\right) .
\end{aligned}
$$

Also, by Assumptions 4, 6, and 11, we have

$$
T^{-1} \bar{G} \bar{G}^{\top}=T^{-1}(\eta \bar{V}+\bar{Z})(\eta \bar{V}+\bar{Z})^{\top} \stackrel{p}{\longrightarrow} \eta \Sigma^{v} \eta^{\top}+\Sigma^{z}
$$

hence it follows that $\widehat{\mathrm{R}}_{g}^{2} \stackrel{p}{\longrightarrow} \mathrm{R}_{g}^{2}$.

Proof of Theorem 4. We denote the estimators of $\bar{V}$ and $\beta$ based on $\breve{p}$ as $\breve{V}$ and $\breve{\beta}$ respectively. Without loss of generality, we can assume $\widehat{p}=p$.

Consider the singular value decomposition of $n^{-1 / 2} T^{-1 / 2} \bar{R}$ by scaling (A.58), we have

$$
n^{-1 / 2} T^{-1 / 2} \varsigma_{p+1: \breve{p}}^{\top} \bar{R}=\Lambda_{p+1: \breve{p}}^{1 / 2} \xi_{p+1: \breve{p}}^{\top} \quad \text { and } \quad n^{-1 / 2} T^{-1 / 2} \bar{R} \xi_{p+1: \breve{p}}=\varsigma_{p+1: \breve{p}} \Lambda_{p+1: \breve{p}}^{1 / 2}
$$

where $\Lambda_{p+1: \breve{p}}$ is a $(\breve{p}-p) \times(\breve{p}-p)$ diagonal matrix with the $i$ th entry on the diagonal being $n^{-1} T^{-1} \lambda_{i}\left(\bar{R}^{\top} \bar{R}\right)$, $\xi_{p+1: \breve{p}}=\left(\xi_{p+1}: \xi_{p+2}: \cdots: \xi_{\breve{p}}\right)$ is $T \times(\breve{p}-p)$, and $\varsigma_{p+1: \breve{p}}=\left(\varsigma_{p+1}: \varsigma_{p+2}: \ldots: \varsigma_{\breve{p}}\right)$ is $n \times(\breve{p}-p)$. It is also easy to observe that

$$
\breve{V}^{\top}=\left(\widehat{V}^{\top}: T^{1 / 2} \xi_{p+1: \breve{p}}\right), \quad \breve{\beta}=\left(\widehat{\beta}: n^{1 / 2} \varsigma_{p+1: \breve{p}} \Lambda_{p+1: \breve{p}}^{1 / 2}\right), \quad \widehat{V} \xi_{p+1: \breve{p}}=0, \quad \text { and } \quad \varsigma_{p+1: \breve{p}}^{\top} \widehat{\beta}=0
$$

Using the formula of block-diagonal matrix inversion, we can decompose

$$
\begin{aligned}
& \breve{\Gamma}-\widehat{\Gamma}=\left(\begin{array}{cc}
1 & \\
& \widehat{\eta}
\end{array}\right)\left\{\frac{1}{n}\left(\begin{array}{ll}
\iota_{n}^{\top} \iota_{n} & \iota_{n}^{\top} \widehat{\beta} \\
\widehat{\beta}^{\top} \iota_{n} & \widehat{\beta}^{\top} \widehat{\beta}
\end{array}\right)\right\}^{-1} \\
& \times\left(\begin{array}{c}
-n^{-1} \iota_{n}^{\top} \varsigma_{p+1: \breve{p}} \Lambda_{p+1: \breve{p}}^{1 / 2} \Delta^{-1} \Lambda_{p+1: \breve{p}}^{1 / 2} \varsigma_{p+1: \breve{p}}^{\top}\left(\mathbb{I}_{n}-\iota_{n}\left(\iota_{n}^{\top} \mathbb{M}_{\widehat{\beta}} \iota_{n}\right)^{-1} \iota_{n}^{\top} \mathbb{M}_{\widehat{\beta}}\right) \bar{r} \\
0
\end{array}\right)
\end{aligned}
$$




$$
+\left(\begin{array}{c}
0 \\
T^{-1 / 2} n^{-1 / 2} \bar{G} \xi_{p+1: \breve{p}} \Delta^{-1} \Lambda_{p+1: \breve{p}_{p+1: \breve{p}}^{\top / 2}}^{\top}\left(\mathbb{I}_{n}-\iota_{n}\left(\iota_{n}^{\top} \mathbb{M}_{\widehat{\beta}} \iota_{n}\right)^{-1} \iota_{n}^{\top} \mathbb{M}_{\widehat{\beta}}\right) \bar{r}
\end{array}\right)
$$

where $\Delta=\Lambda_{p+1: \breve{p}}^{1 / 2}\left(\mathbb{I}_{\breve{p}-p}-\varsigma_{p+1: \breve{p}}^{\top} \iota_{n}\left(\iota_{n}^{\top} \mathbb{M}_{\widehat{\beta}} \iota_{n}\right)^{-1} \iota_{n}^{\top} \varsigma_{p+1: \breve{p}}\right) \Lambda_{p+1: \breve{p}}^{1 / 2}$.

We analyze the right-hand side terms in the following. First, since $\mathbb{M}_{\widehat{\beta}} \widehat{\beta}=0$ and $\varsigma_{p+1: \bar{p}} \widehat{\beta}=0$, we have

$$
\varsigma_{p+1: \breve{p}}^{\top}\left(\mathbb{I}_{n}-\iota_{n}\left(\iota_{n}^{\top} \mathbb{M}_{\widehat{\beta}} \iota_{n}\right)^{-1} \iota_{n}^{\top} \mathbb{M}_{\widehat{\beta}}\right) \bar{r}=\varsigma_{p+1: \breve{p}}^{\top}\left(\mathbb{I}_{n}-\iota_{n}\left(\iota_{n}^{\top} \mathbb{M}_{\widehat{\beta}} \iota_{n}\right)^{-1} \iota_{n}^{\top} \mathbb{M}_{\widehat{\beta}}\right)(\alpha+(\beta-\widehat{\beta} H)(\bar{v}+\gamma)+\bar{u})
$$

Since $\alpha$ is independent of $\varsigma_{p+1: \breve{p}}$, we have

$$
\mathrm{E}\left\|\varsigma_{p+1: \breve{p}}^{\top} \alpha\right\|_{\mathrm{F}}^{2}=\sum_{j=1}^{\breve{p}-p} \mathrm{E}\left(\sum_{i=1}^{n} \varsigma_{p+1: \breve{p}, i j} \alpha_{i}\right)^{2}=\left\|\varsigma_{p+1: \breve{p}}\right\|_{\mathrm{F}}^{2}\left(\sigma^{\alpha}\right)^{2} \leq K,
$$

hence $\left\|\varsigma_{p+1: \breve{p}}^{\top} \alpha\right\|_{\mathrm{F}}=O_{p}(1)$. By Lemma 3(b) and (A.2), we have

$$
\left\|\varsigma_{p+1: \breve{p}}^{\top}(\beta-\widehat{\beta} H)\right\|=O_{p}\left(1+n^{1 / 2} T^{-1 / 2}\right), \quad\left\|\varsigma_{p+1: \bar{p}}^{\top} \bar{u}\right\|=O_{p}\left(n^{1 / 2} T^{-1 / 2}\right) .
$$

On the other hand, since by Lemmas $6,8,(\mathrm{~A} .21)$, we have

$$
\left\|\iota_{n}^{\top} \mathbb{M}_{\widehat{\beta}} \alpha\right\|=O_{p}\left(n^{1 / 2}\right), \quad\left(\iota_{n}^{\top} \mathbb{M}_{\widehat{\beta} \iota_{n}}\right)^{-1}=O_{p}\left(n^{-1}\right) .
$$

By Lemmas 4 and 5, we have

$$
\left\|\iota_{n}^{\top} \mathbb{M}_{\widehat{\beta}} \bar{u}\right\|=O_{p}\left(1+n T^{-1}\right), \quad\left\|\iota_{n}^{\top} \mathbb{M}_{\widehat{\beta}}(\beta-\widehat{\beta} H)\right\|=O_{p}\left(1+n T^{-1}\right) .
$$

Combined with Lemma 9, it implies that

$$
\left\|\varsigma_{p+1: \breve{p}}^{\top} \iota_{n}\left(\iota_{n}^{\top} \mathbb{M}_{\widehat{\beta}} \iota_{n}\right)^{-1}\left(\iota_{n}^{\top} \mathbb{M}_{\widehat{\beta}}(\alpha+(\beta-\widehat{\beta} H)(\bar{v}+\gamma)+\bar{u})\right)\right\|=o_{p}\left(1+n^{1 / 2} T^{-1}\right) .
$$

With the above estimates, we obtain

$$
\begin{aligned}
& \left\|\varsigma_{p+1: \breve{p}}^{\top}\left(\mathbb{I}_{n}-\iota_{n}\left(\iota_{n}^{\top} \mathbb{M}_{\widehat{\beta}} \iota_{n}\right)^{-1} \iota_{n}^{\top} \mathbb{M}_{\widehat{\beta}}\right) \bar{r}\right\| \\
\leq & \left\|\varsigma_{p+1: \breve{p}}^{\top}(\alpha+(\beta-\widehat{\beta} H)(\bar{v}+\gamma)+\bar{u})\right\|+\left\|\varsigma_{p+1: \breve{p}}^{\top} \iota_{n}\left(\iota_{n}^{\top} \mathbb{M}_{\widehat{\beta}} \iota_{n}\right)^{-1}\left(\iota_{n}^{\top} \mathbb{M}_{\widehat{\beta}}(\alpha+(\beta-\widehat{\beta} H)(\bar{v}+\gamma)+\bar{u})\right)\right\| \\
= & O_{p}\left(1+n^{1 / 2} T^{-1 / 2}\right) .
\end{aligned}
$$

Next, by Sherman-Morrison-Woodbury formula, we have

$$
\begin{aligned}
\left\|\Lambda_{p+1: \breve{p}}^{1 / 2} \Delta^{-1} \Lambda_{p+1: \breve{p}}^{1 / 2}\right\| & =\left\|\mathbb{I}_{\breve{p}-p}+\varsigma_{p+1: \breve{p}}^{\top} \iota_{n} \iota_{n}^{\top} \varsigma_{p+1: \breve{p}}\left(\iota_{n}^{\top} \mathbb{M}_{\widehat{\beta}} \iota_{n}-\iota_{n}^{\top} \varsigma_{p+1: \breve{p}} \varsigma_{p+1: \breve{p}}^{\top} \breve{l}_{n}\right)^{-1}\right\| \\
& =\left\|\mathbb{I}_{\breve{p}-p}+n^{-1} \varsigma_{p+1: \breve{p}}^{\top} \iota_{n} \iota_{n}^{\top} \varsigma_{p+1: \breve{p}}\left(1-n^{-1} \iota_{n}^{\top} \varsigma_{1: \breve{p}} \varsigma_{1: \breve{p}}^{\top} \iota_{n}\right)^{-1}\right\|,
\end{aligned}
$$


where we use the fact that

$$
n^{-1} \iota_{n}^{\top} \mathbb{M}_{\widehat{\beta} \iota_{n}}=1-n^{-1} \iota_{n}^{\top} \widehat{\beta}\left(\widehat{\beta}^{\top} \widehat{\beta}\right)^{-1} \widehat{\beta}^{\top} \iota_{n}=1-n^{-1} \iota_{n}^{\top} \varsigma_{1: p} \varsigma_{1: p}^{\top} \iota_{n} .
$$

By Lemma 9, for any $\breve{p} \leq K$, we have

$$
\left(1-n^{-1} \iota_{n}^{\top} \varsigma_{1: \breve{s}} \varsigma_{1: \breve{p}}^{\top} \iota_{n}\right)^{-1}=\left(1-n^{-1} \iota_{n}^{\top} \varsigma_{1: p} \varsigma_{1: p}^{\top} \iota_{n}+o_{p}(1)\right)^{-1}=\left(1-\beta_{0}^{\top}\left(\Sigma^{\beta}\right)^{-1} \beta_{0}\right)^{-1}+o_{p}(1) .
$$

Also, $\left\|n^{-1} \varsigma_{p+1: \breve{p}}^{\top} \iota_{n} \iota_{n}^{\top} \varsigma_{p+1: \breve{p}}\right\|=o_{p}(1)$, which in turn leads to

$$
\left\|\Lambda_{p+1: \breve{p}}^{1 / 2} \Delta^{-1} \Lambda_{p+1: \breve{p}}^{1 / 2}\right\|=O_{p}(1)
$$

Combining (A.23), (A.24), and using Lemma 9 again, we have

$$
\left\|n^{-1} \iota_{n}^{\top} \varsigma_{p+1: \breve{p}} \Lambda_{p+1: \breve{p}}^{1 / 2} \Delta^{-1} \Lambda_{p+1: \breve{p}}^{1 / 2} \varsigma_{p+1: \breve{p}}^{\top}\left(\mathbb{I}_{n}-\iota_{n}\left(\iota_{n}^{\top} \mathbb{M}_{\widehat{\beta}} \iota_{n}\right)^{-1} \iota_{n}^{\top} \mathbb{M}_{\widehat{\beta}}\right) \bar{r}\right\|=o_{p}\left(n^{-1 / 2}+T^{-1 / 2}\right) .
$$

Next, by Lemmas 1 and 2,

$$
\left\|\eta \bar{V} \xi_{p+1: \breve{p}}\right\|=\left\|\eta\left(\bar{V}-H^{-1} \widehat{V}\right) \xi_{p+1: \breve{p}}\right\|=O_{p}\left(1+n^{-1 / 2} T^{1 / 2}\right)
$$

By the independence of $\bar{Z}$ and $\xi_{p+1: \breve{p}}$, we also have $\left\|\bar{Z} \xi_{p+1: \breve{p}}\right\|=O_{p}(1)$. On the other hand, it follows from (A.15) that

$$
\bar{R} \bar{R}^{\top}+\bar{U} \bar{V}^{\top}\left(\bar{V} \bar{V}^{\top}\right)^{-1} \bar{V} \bar{U}^{\top}=\bar{U} \bar{U}^{\top}+\tilde{\beta} \bar{V} \bar{V}^{\top} \tilde{\beta}^{\top}
$$

where $\tilde{\beta}=\beta+U \bar{V}^{\top}\left(\bar{V} \bar{V}^{\top}\right)^{-1}$. By (4.3.2a) and (4.3.2b) of Theorem 4.3.1 in Horn and Johnson (2013), for $p+1 \leq j \leq \breve{p}$,

$\lambda_{j+p}\left(\bar{U} \bar{U}^{\top}\right)+\lambda_{n-1}\left(\tilde{\beta} \bar{V} \bar{V}^{\top} \tilde{\beta}^{\top}\right) \leq \lambda_{j+p}\left(\bar{R} \bar{R}^{\top}+\bar{U} \bar{V}^{\top}\left(\bar{V} \bar{V}^{\top}\right)^{-1} \bar{V} \bar{U}^{\top}\right) \leq \lambda_{j}\left(\bar{R} \bar{R}^{\top}\right)+\lambda_{p+1}\left(\bar{U} \bar{V}^{\top}\left(\bar{V} \bar{V}^{\top}\right)^{-1} \bar{V} \bar{U}^{\top}\right)$.

Since $\operatorname{rank}\left(\tilde{\beta} \bar{V} \bar{V}^{\top} \tilde{\beta}^{\top}\right) \leq p$ and $\operatorname{rank}\left(\bar{U} \bar{V}^{\top}\left(\bar{V} \bar{V}^{\top}\right)^{-1} \bar{V} \bar{U}^{\top}\right) \leq p$, we obtain,

$$
\lambda_{\breve{p}+p}\left(\bar{U} \bar{U}^{\top}\right) \leq \lambda_{j+p}\left(\bar{U} \bar{U}^{\top}\right) \leq \lambda_{j}\left(\bar{R} \bar{R}^{\top}\right) \leq \lambda_{j-p}\left(\bar{U} \bar{U}^{\top}\right) \leq \lambda_{1}\left(\bar{U} \bar{U}^{\top}\right)
$$

Since the empirical distribution of eigenvalues of $T^{-1} \bar{U} \bar{U}^{\top}$ follows the Marčenko-Pastur law (see, e.g., Theorem 3.6 in Bai and Silverstein (2009)), as $n / T \rightarrow c \in(0, \infty)$, and this limiting law has a bounded support, then there exist upper and lower bounds for finitely many largest eigenvalues

$$
0<K^{\prime} \leq T^{-1} \lambda_{j}\left(\bar{R} \bar{R}^{\top}\right) \leq K, \quad p+1 \leq j \leq \breve{p}
$$


so that $\left\|\Lambda_{p+1: \breve{p}}^{-1 / 2}\right\|=O_{p}\left(n^{1 / 2}\right)$. We therefore obtain that

$$
\begin{aligned}
& \left\|T^{-1 / 2} n^{-1 / 2} \bar{G} \xi_{p+1: \breve{p}} \Delta^{-1} \Lambda_{p+1: \breve{p}}^{1 / 2} \varsigma_{p+1: \breve{p}}^{\top}\left(\mathbb{I}_{n}-\iota_{n}\left(\iota_{n}^{\top} \mathbb{M}_{\widehat{\beta}} \iota_{n}\right)^{-1} \iota_{n}^{\top} \mathbb{M}_{\widehat{\beta}}\right) \bar{r}\right\| \\
\leq & \left\|T^{-1 / 2} n^{-1 / 2} \bar{G} \xi_{p+1: \breve{p}}\right\|\left\|\Lambda_{p+1: \breve{p}}^{-1 / 2}\right\|\left\|\Lambda_{p+1: \breve{p}}^{1 / 2} \Delta^{-1} \Lambda_{p+1: \breve{p}}^{1 / 2}\right\|\left\|\varsigma_{p+1: \breve{p}}^{\top}\left(\mathbb{I}_{n}-\iota_{n}\left(\iota_{n}^{\top} \mathbb{M}_{\widehat{\beta}} \iota_{n}\right)^{-1} \iota_{n}^{\top} \mathbb{M}_{\widehat{\beta}}\right) \bar{r}\right\| \\
= & O_{p}\left(n^{-1 / 2}\right),
\end{aligned}
$$

which conclude the proof.

Proof of Theorem 5. For any $1 \leq t \leq T$, we have

$$
\widehat{g}_{t}-\eta v_{t}=\left(\widehat{\eta}-\eta H^{-1}\right)\left(\widehat{v}_{t}-H \bar{v}_{t}\right)+\left(\widehat{\eta}-\eta H^{-1}\right) H \bar{v}_{t}+\eta H^{-1}\left(\widehat{v}_{t}-H \bar{v}_{t}\right)-\eta \bar{v}
$$

By (A.37), we have

$$
\begin{aligned}
\widehat{v}_{t}-H \bar{v}_{t}= & n^{-1} T^{-1} \widehat{\Lambda}^{-1}(\widehat{V}-H \bar{V})\left(\bar{U}^{\top} \beta \bar{v}_{t}+\bar{U}^{\top} \bar{u}_{t}\right)+n^{-1} T^{-1} \widehat{\Lambda}^{-1}\left(H \bar{V} \bar{U}^{\top} \beta \bar{v}_{t}+H \bar{V} \bar{U}^{\top} \bar{u}_{t}\right) \\
& +n^{-1} T^{-1} \widehat{\Lambda}^{-1} \widehat{V} \bar{V}^{\top} \beta^{\top} \bar{u}_{t} .
\end{aligned}
$$

By Assumption 3(ii), we have

$$
\mathrm{E}\left\|\beta^{\top} u_{t}\right\|_{\mathrm{F}}^{2}=\mathrm{E} \sum_{i=1}^{p}\left(\sum_{k=1}^{n} \beta_{k i} u_{k t}\right)^{2} \leq K \sum_{k=1}^{n} \sum_{k^{\prime}=1}^{n}\left|\sigma_{k k^{\prime}, t}\right| \leq K n,
$$

so that

$$
\left\|\beta^{\top} \bar{u}_{t}\right\|_{\mathrm{F}} \leq\left\|\beta^{\top} u_{t}\right\|_{\mathrm{F}}+\left\|\beta^{\top} \bar{u}\right\|_{\mathrm{F}}=O_{p}\left(n^{1 / 2}\right)
$$

By Assumption 3(i)(iv) and Assumption 12, using the fact that $\left|\rho_{n, s t}\right| \leq 1$, we have

$$
\begin{aligned}
\mathrm{E}\left\|U^{\top} u_{t}\right\|_{\mathrm{F}}^{2} & =\mathrm{E} \sum_{s=1}^{T}\left(n \gamma_{n, s t}+\sum_{k=1}^{n}\left(u_{k s} u_{k t}-\mathrm{E}\left(u_{k s} u_{k t}\right)\right)\right)^{2} \\
& \leq K n^{2} \sum_{s=1}^{T} \gamma_{n, s t}^{2}+K n T \leq n^{2} \sum_{s=1}^{T}\left|\gamma_{n, s t}\right|+K n T=K n^{2}+K n T \\
\mathrm{E}\left\|u_{t}\right\|_{\mathrm{F}}^{2} & \leq \sum_{k=1}^{n} \mathrm{E} u_{k t}^{2} \leq \sum_{k=1}^{n}\left|\sigma_{k k^{\prime}}\right| \leq K .
\end{aligned}
$$

Then from (A.2) and (A.54), it follows that

$$
\left\|\bar{U}^{\top} \bar{u}_{t}\right\|_{\mathrm{F}} \leq\left\|\bar{U}^{\top} \bar{u}\right\|_{\mathrm{F}}+\left\|U^{\top} u_{t}\right\|_{\mathrm{F}}+\left\|\iota_{T}\right\|_{\mathrm{F}}\left\|\bar{u}^{\top}\right\|_{\mathrm{F}}\left\|u_{t}\right\|_{\mathrm{F}}=O_{p}\left(n+n^{1 / 2} T^{1 / 2}\right) .
$$


The above estimates, along with (A.9), Lemma 1, and $\left\|\bar{v}_{t}\right\|=O_{p}(1)$, lead to

$$
\begin{aligned}
& \left\|n^{-1} T^{-1} \widehat{\Lambda}^{-1}(\widehat{V}-H \bar{V})\left(\bar{U}^{\top} \beta \bar{v}_{t}+\bar{U}^{\top} \bar{u}_{t}\right)\right\|_{\mathrm{MAX}} \\
\leq & n^{-1} T^{-1}\left\|\widehat{\Lambda}^{-1}\right\|_{\mathrm{MAX}}\|\widehat{V}-H \bar{V}\|_{\mathrm{F}}\left(\left\|\bar{U}^{\top} \beta\right\|_{\mathrm{F}}\left\|\bar{v}_{t}\right\|+\left\|\bar{U}^{\top} \bar{u}_{t}\right\|_{\mathrm{F}}\right)=O_{p}\left(n^{-1}+T^{-1}\right) .
\end{aligned}
$$

Moreover, it follows from (A.2), (A.44), and (A.49) that

$$
\begin{aligned}
& \left\|n^{-1} T^{-1} \widehat{\Lambda}^{-1}\left(H \bar{V} \bar{U}^{\top} \beta \bar{v}_{t}+H \bar{V} \bar{U}^{\top} \bar{u}_{t}\right)\right\|_{\mathrm{MAX}} \\
\leq & K n^{-1} T^{-1}\left\|\widehat{\Lambda}^{-1}\right\|_{\mathrm{MAX}}\|H\|\left(\left\|\bar{V} \bar{U}^{\top} \beta\right\|_{\mathrm{MAX}}\left\|\bar{v}_{t}\right\|+\left\|\bar{V} \bar{U}^{\top}\right\|_{\mathrm{F}}\left(\left\|u_{t}\right\|_{\mathrm{F}}+\|u\|_{\mathrm{F}}\right)\right) \\
= & O_{p}\left(n^{-1 / 2} T^{-1 / 2}+T^{-1}\right) .
\end{aligned}
$$

We thereby focus on the remaining term, which by Lemma 1, (A.11) and (A.27), satisfies

$$
n^{-1} T^{-1}\left\|\widehat{\Lambda}^{-1} \widehat{V} \bar{V}^{\top} \beta^{\top} \bar{u}_{t}\right\|_{\mathrm{MAX}} \leq K n^{-1} T^{-1}\left\|\widehat{\Lambda}^{-1}\right\|_{\mathrm{MAX}}\|\widehat{V}\|_{\mathrm{F}}\left\|\bar{V}^{\top}\right\|_{\mathrm{F}}\left\|\beta^{\top} \bar{u}_{t}\right\|_{\mathrm{MAX}}=O_{p}\left(n^{-1 / 2}\right) .
$$

Therefore, we have

$$
\left\|\widehat{v}_{t}-H \bar{v}_{t}\right\|_{\mathrm{MAX}}=O_{p}\left(n^{-1 / 2}+T^{-1}\right)
$$

Then by (A.25), (A.26), and (A.19), we have

$$
\left\|\widehat{g}_{t}-\eta v_{t}-\left(T^{-1} \bar{Z} \bar{V}^{\top} H^{\top} H v_{t}+n^{-1} T^{-1} \eta H^{-1} \widehat{\Lambda}^{-1} \widehat{V} \bar{V}^{\top} \beta^{\top} u_{t}-\eta \bar{v}\right)\right\|_{\mathrm{MAX}}=o_{p}\left(n^{-1 / 2}+T^{-1 / 2}\right) .
$$

Next, we note that by Assumption 11 and Lemma 2,

$$
\begin{aligned}
& T^{1 / 2}\left(\begin{array}{c}
T^{-1} \operatorname{vec}\left(\bar{Z} \bar{V}^{\top} H^{\top} H v_{t}\right) \\
\eta \bar{v}
\end{array}\right)=T^{1 / 2}\left(\begin{array}{c}
\left(v_{t}^{\top} H^{\top} H \otimes \mathbb{I}_{d}\right) \operatorname{vec}\left(\bar{Z} \bar{V}^{\top}\right) \\
\eta \bar{v}
\end{array}\right) \\
& \stackrel{\mathcal{L}}{\longrightarrow} \mathcal{N}\left(0,\left(\begin{array}{cc}
\left(v_{t}^{\top}\left(\Sigma^{v}\right)^{-1} \otimes \mathbb{I}_{d}\right) \Pi_{11}\left(\left(\Sigma^{v}\right)^{-1} v_{t} \otimes \mathbb{I}_{d}\right) & \left(v_{t}^{\top}\left(\Sigma^{v}\right)^{-1} \otimes \mathbb{I}_{d}\right) \Pi_{12} \eta^{\top} \\
. & \eta \Pi_{22} \eta^{\top}
\end{array}\right)\right) .
\end{aligned}
$$

By (A.17) and Assumptions 5 and 13, we have

$$
n^{-1 / 2} T^{-1} \eta H^{-1} \widehat{\Lambda}^{-1} \widehat{V} \bar{V}^{\top} \beta^{\top} u_{t}=n^{1 / 2} \eta\left(\beta^{\top} \beta\right)^{-1} \beta^{\top} u_{t} \stackrel{\mathcal{L}}{\longrightarrow} \mathcal{N}\left(0, \eta\left(\Sigma^{\beta}\right)^{-1} \Omega_{t}\left(\Sigma^{\beta}\right)^{-1} \eta^{\top}\right) .
$$

The desired result follows from the same asymptotic independence argument as in Bai (2003).

Proof of Theorem 6. Again, we assume $\widehat{p}=p$. To prove the consistency of $\widehat{\Phi}$, without loss of generality, we focus on the case of $\Pi_{12}$, and show that

$$
\left(\widetilde{\gamma}^{\top} \otimes \mathbb{I}_{d}\right) \widehat{\Pi}_{12} \widehat{\eta}^{\top} \stackrel{p}{\longrightarrow}\left(\gamma^{\top}\left(\Sigma^{v}\right)^{-1} \otimes \mathbb{I}_{d}\right) \Pi_{12} \eta^{\top} .
$$


The proof for the other two terms in $\widehat{\Phi}$ is similar and hence is omitted.

Note that by (A.53), Lemma 2, Lemma 3(a), and Assumption 4, we have

$$
\begin{aligned}
& \left\|T^{-1} H^{-1} \widehat{V} \widehat{V}^{\top} H^{-\top}-\Sigma^{v}\right\|_{\text {MAX }} \\
= & \left\|T^{-1} H^{-1}(\widehat{V}-H \bar{V}) \widehat{V}^{\top} H^{-\top}+T^{-1} \bar{V}\left(\widehat{V}^{\top}-\bar{V}^{\top} H^{\top}\right) H^{-\top}+T^{-1} V V^{\top}-\Sigma^{v}-\bar{v} \bar{v}^{\top}\right\|_{\text {MAX }} \\
= & O_{p}\left(n^{-1}+T^{-1 / 2}\right) .
\end{aligned}
$$

By (A.20), Lemma 2, and the proof of Theorem 2, we have

$$
\|\widehat{\eta} H-\eta\|_{\mathrm{MAX}}=O_{p}\left(n^{-1}+T^{-1 / 2}\right), \quad\left\|H^{-1} \widetilde{\gamma}-\gamma\right\|_{\mathrm{MAX}}=O_{p}\left(n^{-1 / 2}+T^{-1 / 2}\right) .
$$

Therefore, to prove (A.30), we only need to show that

$$
\widetilde{\Pi}_{12}:=\left(H^{-1} \otimes \mathbb{I}_{d}\right) \widehat{\Pi}_{12} H^{-\top} \stackrel{p}{\longrightarrow} \Pi_{12},
$$

with which, and by the continuous mapping theorem, we have

$$
\begin{aligned}
\left(\widetilde{\gamma}^{\top}\left(\widehat{\Sigma}^{v}\right)^{-1} \otimes \mathbb{I}_{d}\right) \widehat{\Pi}_{12} \widehat{\eta}^{\top} & =\left(\left(H^{-1} \widetilde{\gamma}\right)^{\top}\left(H^{-1} \widehat{\Sigma}^{v} H^{-\top}\right)^{-1} \otimes \mathbb{I}_{d}\right)\left(H^{-1} \otimes \mathbb{I}_{d}\right) \widehat{\Pi}_{12} H^{-\top}(\widehat{\eta} H)^{\top} \\
& \stackrel{p}{\longrightarrow}\left(\gamma^{\top}\left(\Sigma^{v}\right)^{-1} \otimes \mathbb{I}_{d}\right) \Pi_{12} \eta^{\top} .
\end{aligned}
$$

Writing $\widetilde{V}=H^{-1} \widehat{V}$, we have

$\widetilde{\Pi}_{12,(i-1) d+j, i^{\prime}}=\operatorname{vec}\left(e_{j} e_{i}^{\top}\right)^{\top}\left(H^{-1} \otimes \mathbb{I}_{d}\right) \widehat{\Pi}_{12} H^{-\top} e_{i^{\prime}}=\operatorname{vec}\left(e_{j} e_{i}^{\top} H^{-1}\right)^{\top} \widehat{\Pi}_{12} H^{-\top} e_{i^{\prime}}=T^{-1} \sum_{t=1}^{T} \sum_{s=1}^{T} \widehat{z}_{j t} \widetilde{v}_{i t} Q_{t s} \widetilde{v}_{i^{\prime} s}$,

where $Q_{s t}=\left(1-\frac{|s-t|}{q+1}\right) 1_{|s-t| \leq q}$.

In fact, to show (A.32), by Lemma 2 we only need to prove for any fixed $1 \leq i, i^{\prime} \leq p$, and $1 \leq j, j^{\prime} \leq d$

$$
\widetilde{\Pi}_{12,(i-1) d+j, i^{\prime}}-T^{-1} \sum_{t=1}^{T} \sum_{s=1}^{T} z_{j t} v_{i t} Q_{t s} v_{i^{\prime} s} \stackrel{p}{\longrightarrow} 0,
$$

since by the identical proof of Theorem 2 in Newey and West (1987), we have

$$
T^{-1} \sum_{t=1}^{T} \sum_{s=1}^{T} z_{j t} v_{i t} Q_{t s} v_{i^{\prime} s}-\Pi_{12,(i-1) d+j, i^{\prime}} \stackrel{p}{\longrightarrow} 0 .
$$

Note that

the left-hand side of (A.33) 


$$
\begin{aligned}
= & T^{-1} \sum_{t=1}^{T} \sum_{s=1}^{T}\left\{\left(\widehat{z}_{j t}-z_{j t}\right)\left(\widetilde{v}_{i t}-v_{i t}\right) Q_{t s}\left(\widetilde{v}_{i^{\prime} s}-v_{i^{\prime} s}\right)+\left(\widehat{z}_{j t}-z_{j t}\right)\left(\widetilde{v}_{i t}-v_{i t}\right) Q_{t s} v_{i^{\prime} s}\right. \\
& \left.+\left(\widehat{z}_{j t}-z_{j t}\right) v_{i t} Q_{t s} \widetilde{v}_{i^{\prime} s}+z_{j t}\left(\widetilde{v}_{i t}-v_{i t}\right) Q_{t s} \widetilde{v}_{i^{\prime} s}+z_{j t} \widetilde{v}_{i t} Q_{t s}\left(\widetilde{v}_{i^{\prime} s}-v_{i^{\prime} s}\right)\right\} .
\end{aligned}
$$

We analyze these terms one by one. Since we have

$$
\widehat{Z}-\bar{Z}=\eta \bar{V}-\widehat{\eta} \widehat{V}=\left(\eta H^{-1}-\widehat{\eta}\right) H \bar{V}-\left(\widehat{\eta}-\eta H^{-1}\right)(\widehat{V}-H \bar{V})-\eta H^{-1}(\widehat{V}-H \bar{V}),
$$

it follows from (A.20), (A.38), and Lemmas 1 and 2 that

$$
\begin{aligned}
& T^{-1}\|\widehat{Z}-\bar{Z}\|_{\mathrm{F}} \\
\leq & K T^{-1}\left(\left\|\eta H^{-1}-\widehat{\eta}\right\|_{\mathrm{MAX}}\|H\|\|\bar{V}\|_{\mathrm{F}}+\left\|\widehat{\eta}-\eta H^{-1}\right\|_{\mathrm{F}}\|\widehat{V}-H \bar{V}\|_{\mathrm{F}}+\left\|\eta H^{-1}\right\|\|\widehat{V}-H \bar{V}\|_{\mathrm{F}}\right) \\
= & O_{p}\left(n^{-1 / 2} T^{-1 / 2}+T^{-1}\right) .
\end{aligned}
$$

Moreover, by Lemma 10(i), Assumption 14, (A.34), and (A.31), we have

$$
\begin{aligned}
& \|\widehat{Z}-\bar{Z}\|_{\text {MAX }} \\
\leq & \left\|\eta H^{-1}-\widehat{\eta}\right\|_{\text {MAX }}\|H\|\|\bar{V}\|_{\text {MAX }}+\left\|\widehat{\eta}-\eta H^{-1}\right\|_{\text {MAX }}\|\widehat{V}-H \bar{V}\|_{\text {MAX }}+\left\|\eta H^{-1}\right\|\|\widehat{V}-H \bar{V}\|_{\text {MAX }} \\
= & O_{p}\left((\log T)^{1 / a} T^{-1 / 2}+n^{-1 / 2} T^{1 / 4}\right) .
\end{aligned}
$$

By Cauchy-Schwartz inequality, Lemmas 1, 10(i), and using the fact that $\left|Q_{t s}\right| \leq 1_{|t-s| \leq q}$ and $\left\|\bar{v} \iota_{T}^{\top}\right\|_{\mathrm{F}}=$ $\|\bar{v}\|_{\mathrm{F}}\left\|\iota_{T}^{\top}\right\|_{\mathrm{F}} \leq K T^{1 / 2}\|\bar{v}\|_{\mathrm{MAX}}=O_{p}(1)$, we have

$$
\begin{aligned}
& \left|T^{-1} \sum_{t=1}^{T} \sum_{s=1}^{T}\left(\widehat{z}_{j t}-z_{j t}\right)\left(\widetilde{v}_{i t}-v_{i t}\right) Q_{t s}\left(\widetilde{v}_{i^{\prime} s}-v_{i^{\prime} s}\right)\right| \\
\leq & K q T^{-1}\left(\|\widetilde{V}-\bar{V}\|_{\mathrm{MAX}}+\left\|\bar{v} \iota_{T}^{\top}\right\|_{\mathrm{MAX}}\right)\left(\|\widetilde{V}-\bar{V}\|_{\mathrm{F}}+\left\|\bar{v} \iota_{T}^{\top}\right\|_{\mathrm{F}}\right)\left(\|\widehat{Z}-\bar{Z}\|_{\mathrm{F}}+\left\|\bar{z} \iota_{T}^{\top}\right\|_{\mathrm{F}}\right) \\
= & O_{p}\left(q\left(T^{-1}+n^{-1}\right)\left(T^{1 / 4} n^{-1 / 2}+T^{-1}\right)\right) .
\end{aligned}
$$

Similarly, because of $\|\widetilde{V}\|_{\mathrm{F}} \leq O_{p}\left(T^{1 / 2}\right)$ implied by (A.39), $\|Z\|_{\mathrm{MAX}}=O_{p}\left((\log T)^{1 / a}\right)$ by Assumption 14 and Lemma 2, and by Assumptions 4 and 6, we have

$$
\begin{aligned}
& \left|T^{-1} \sum_{t=1}^{T} \sum_{s=1}^{T}\left(\widehat{z}_{j t}-z_{j t}\right)\left(\widetilde{v}_{i t}-v_{i t}\right) Q_{t s} v_{i^{\prime} s}\right| \\
\leq & K q T^{-1}\|V\|_{\mathrm{MAX}}\left(\|\widetilde{V}-\bar{V}\|_{\mathrm{F}}+\left\|\bar{v} \iota_{T}^{\top}\right\|_{\mathrm{F}}\right)\left(\|\widehat{Z}-\bar{Z}\|_{\mathrm{F}}+\left\|\bar{z} \iota_{T}^{\top}\right\|_{\mathrm{F}}\right)=O_{p}\left(q(\log T)^{1 / a}\left(n^{-1}+T^{-1}\right)\right), \\
& \left|T^{-1} \sum_{t=1}^{T} \sum_{s=1}^{T}\left(\widehat{z}_{j t}-z_{j t}\right) v_{i t} Q_{t s} \widetilde{v}_{i^{\prime} s}\right| \\
\leq & K q T^{-1}\|V\|_{\mathrm{MAX}}\|\widetilde{V}\|_{\mathrm{F}}\left(\|\widehat{Z}-\bar{Z}\|_{\mathrm{F}}+\left\|\bar{z}_{T}^{\top}\right\|_{\mathrm{F}}\right)=O_{p}\left(q(\log T)^{1 / a}\left(n^{-1 / 2}+T^{-1 / 2}\right)\right),
\end{aligned}
$$




$$
\begin{aligned}
& \left|T^{-1} \sum_{t=1}^{T} \sum_{s=1}^{T} z_{j t}\left(\widetilde{v}_{i t}-v_{i t}\right) Q_{t s} \widetilde{v}_{i^{\prime} s}\right| \\
\leq & K q T^{-1}\|Z\|_{\mathrm{MAX}}\|\widetilde{V}\|_{\mathrm{F}}\left(\left\|H^{-1} \widehat{V}-\bar{V}\right\|_{\mathrm{F}}+\left\|\bar{v} \iota_{T}^{\top}\right\|_{\mathrm{F}}\right)=O_{p}\left(q(\log T)^{1 / a}\left(n^{-1 / 2}+T^{-1 / 2}\right)\right), \\
& \left|T^{-1} \sum_{t=1}^{T} \sum_{s=1}^{T} z_{j t} \widetilde{v}_{i t} Q_{t s}\left(\widetilde{v}_{i^{\prime} s}-v_{i^{\prime} s}\right)\right| \\
\leq & K q T^{-1}\|Z\|_{\mathrm{MAX}}\|\widetilde{V}\|_{\mathrm{F}}\left(\left\|H^{-1} \widehat{V}-\bar{V}\right\|_{\mathrm{F}}+\left\|\bar{v} \iota_{T}^{\mathrm{T}}\right\|_{\mathrm{F}}\right)=O_{p}\left(q(\log T)^{1 / a}\left(n^{-1 / 2}+T^{-1 / 2}\right)\right) .
\end{aligned}
$$

All the above terms converge to 0 , as $T, n \rightarrow \infty$, with $q T^{-1 / 4}+q n^{-1 / 4} \rightarrow 0$ and $n^{-3} T \rightarrow 0$, which establishes (A.33).

Finally, to show the consistency of $\widehat{\Upsilon}$, we first note

$$
\left\|H^{\top}\left(\widehat{\Sigma}^{\beta}-\widehat{\beta}_{0} \widehat{\beta}_{0}^{\top}\right) H-\left(\Sigma^{\beta}-\beta_{0} \beta_{0}^{\top}\right)\right\|_{\mathrm{MAX}} \leq\left\|H^{\top} \widehat{\Sigma}^{\beta} H-\Sigma^{\beta}\right\|_{\mathrm{MAX}}+\left\|H^{\top} \widehat{\beta}_{0} \widehat{\beta}_{0}^{\top} H-\beta_{0} \beta_{0}^{\top}\right\|_{\mathrm{MAX}} .
$$

By Lemmas 2, 4(d), 5(d), and Assumption 5,

$$
\begin{aligned}
& \left\|H^{\top} \widehat{\Sigma}^{\beta} H-\Sigma^{\beta}\right\|_{\text {MAX }} \\
\leq & \left\|n^{-1} H^{\top} \widehat{\beta}^{\top} \widehat{\beta} H-n^{-1} \beta^{\top} \beta\right\|_{\mathrm{MAX}}+\left\|n^{-1} \beta^{\top} \beta-\Sigma^{\beta}\right\|_{\text {MAX }} \\
\leq & \left\|n^{-1}\left(H^{\top} \widehat{\beta}^{\top}-\beta^{\top}\right)(\widehat{\beta} H-\beta)+n^{-1}\left(H^{\top} \widehat{\beta}^{\top}-\beta^{\top}\right) \beta-n^{-1} \beta^{\top}(\beta-\widehat{\beta} H)\right\|_{\text {MAX }}+o_{p}(1) \\
= & o_{p}(1) . \\
& \left\|H^{\top} \widehat{\beta}_{0} \widehat{\beta}_{0}^{\top} H-\beta_{0} \beta_{0}^{\top}\right\|_{\text {MAX }} \\
\leq & \left\|\left(H^{\top} \widehat{\beta}_{0}-\beta_{0}\right)\left(\widehat{\beta}_{0}^{\top} H-\beta_{0}^{\top}\right)+\beta_{0}\left(\widehat{\beta}_{0}^{\top} H-\beta_{0}^{\top}\right)+\left(H^{\top} \widehat{\beta}_{0}-\beta_{0}\right) \beta_{0}^{\top}\right\|_{\text {MAX }} \\
= & o_{p}(1),
\end{aligned}
$$

where we also use Lemma 4(c):

$$
\left\|H^{\top} \widehat{\beta}_{0}-\beta_{0}\right\|_{\mathrm{MAX}}=n^{-1}\left\|\left(H^{\top} \widehat{\beta}^{\top}-\beta^{\top}\right) \iota_{n}\right\|_{\mathrm{MAX}}=o_{p}(1) .
$$

Next, by Lemma 3(b) and (A.31), we have

$$
\begin{aligned}
{\widehat{\sigma^{\alpha}}}^{2}-\left(\sigma^{\alpha}\right)^{2}= & n^{-1}\left\|\bar{r}-\iota_{n} \widetilde{\gamma}_{0}-\widehat{\beta} \widetilde{\gamma}\right\|_{\mathrm{F}}^{2}-\left(\sigma^{\alpha}\right)^{2} \\
= & n^{-1}\left\|\iota_{n}\left(\gamma_{0}-\widetilde{\gamma}_{0}\right)+\beta \gamma-\widehat{\beta} \widetilde{\gamma}+\beta \bar{v}+\bar{u}\right\|_{\mathrm{F}}^{2}+n^{-1}\|\alpha\|_{\mathrm{F}}^{2}-\left(\sigma^{\alpha}\right)^{2} \\
\leq & n^{-1}\left\|\iota_{n}\right\|_{\mathrm{F}}^{2}\left\|\gamma_{0}-\widetilde{\gamma}_{0}\right\|_{\mathrm{F}}^{2}+n^{-1}\|\beta\|_{\mathrm{F}}^{2}\|\bar{v}\|_{\mathrm{F}}^{2}+n^{-1}\|\bar{u}\|_{\mathrm{F}}^{2}+n^{-1}\|(\widehat{\beta} H-\beta) \gamma\|_{\mathrm{F}}^{2} \\
& +n^{-1}\left\|(\widehat{\beta} H-\beta)\left(H^{-1} \widetilde{\gamma}-\gamma\right)\right\|_{\mathrm{F}}^{2}+n^{-1}\left\|\beta\left(H^{-1} \widetilde{\gamma}-\gamma\right)\right\|_{\mathrm{F}}^{2}+o_{p}(1) .
\end{aligned}
$$


Therefore, by (A.20) and the continuous mapping theorem,

$$
{\widehat{\sigma^{\alpha}}}^{2} \widehat{\eta} H H^{-1}\left(\widehat{\Sigma}^{\beta}-\widehat{\beta}_{0} \widehat{\beta}_{0}^{\top}\right)^{-1} H^{-\top} H^{\top} \widehat{\eta}^{\top} \stackrel{p}{\longrightarrow} \Upsilon
$$

which concludes the proof.

Proof of Theorem $\%$. For $\widehat{\Psi}_{1 t}$, we can follow exactly the same proof as that of Theorem 6 , since, similar to (A.31) for $\widetilde{\gamma}$, we have the same estimate for $\widehat{v}_{t}$ by (A.29).

As to $\widehat{\Psi}_{2 t}$, similarly, we only need to show

$$
\left\|H^{\top} \widehat{\Omega}_{t} H-\Omega_{t}\right\|_{\mathrm{MAX}}=o_{p}(1) .
$$

Then by the continuous mapping theorem, along with (A.31) and (A.35), we have

$$
\widehat{\Psi}_{2 t}=\widehat{\eta} H\left(H^{\top} \widehat{\Sigma}^{\beta} H\right)^{-1} H^{\top} \widehat{\Omega}_{t} H\left(H^{\top} \widehat{\Sigma}^{\beta} H\right)^{-1} H^{\top} \widehat{\eta}^{\top} \stackrel{p}{\longrightarrow} \Psi_{2 t} .
$$

We analyze the case with estimator (7) first. Note that

$$
\left\|H^{\top} \widehat{\Omega}_{t} H-\Omega_{t}\right\|_{\mathrm{MAX}} \leq \frac{1}{n}\left\|\sum_{i=1}^{n}\left(H^{\top} \widehat{\beta}_{i} \widehat{\beta}_{i}^{\top} H \widehat{u}_{i t}^{2}-\beta_{i} \beta_{i}^{\top} u_{i t}^{2}\right)\right\|_{\mathrm{MAX}}+\frac{1}{n}\left\|\sum_{i=1}^{n} \beta_{i} \beta_{i}^{\top}\left(u_{i t}^{2}-\mathrm{E} u_{i t}^{2}\right)\right\|_{\mathrm{MAX}} .
$$

On the one hand, by the law of large numbers for i.n.i.d sequences, we have

$$
\left\|\frac{1}{n} \sum_{i=1}^{n} \beta_{i} \beta_{i}^{\top}\left(u_{i t}^{2}-\mathrm{E} u_{i t}^{2}\right)\right\|_{\mathrm{MAX}}=o_{p}(1) .
$$

On the other hand, writing $\widetilde{\beta}=\widehat{\beta} H$, for some fixed $t \leq T$, we first show

$$
\sum_{i=1}^{n}\left(\widehat{u}_{i t}-u_{i t}\right)^{2}=O_{p}\left(1+n T^{-1}\right)
$$

In fact, by (A.29), $\|\beta\|_{\mathrm{F}}=O_{p}\left(n^{1 / 2}\right),\left\|\bar{v}_{t}\right\|=O_{p}(1)$, and Lemma 3(b),

$$
\begin{aligned}
\sum_{i=1}^{n}\left(\widehat{u}_{i t}-\bar{u}_{i t}\right)^{2} & =\sum_{i=1}^{n}\left(\widetilde{\beta}_{i}^{\top} \widetilde{v}_{t}-\beta_{i}^{\top} \bar{v}_{t}\right)^{2} \leq \sum_{i=1}^{n}\left(\left(\widetilde{\beta}_{i}-\beta_{i}\right)^{\top}\left(\widetilde{v}_{t}-\bar{v}_{t}\right)+\beta_{i}^{\top}\left(\widetilde{v}_{t}-\bar{v}_{t}\right)-\left(\beta_{i}-\widetilde{\beta}_{i}\right)^{\top} \bar{v}_{t}\right)^{2} \\
& \leq K\left\|\widetilde{v}_{t}-\bar{v}_{t}\right\|^{2}\left(\|\widetilde{\beta}-\beta\|_{\mathrm{F}}^{2}+\|\beta\|_{\mathrm{F}}^{2}\right)+K\|\widetilde{\beta}-\beta\|_{\mathrm{F}}^{2}\left\|\bar{v}_{t}\right\|^{2} \\
& =O_{p}\left(1+n T^{-1}\right) .
\end{aligned}
$$

Hence, by (A.2) we have

$$
\sum_{i=1}^{n}\left(\widehat{u}_{i t}-u_{i t}\right)^{2} \leq 2 \sum_{i=1}^{n}\left(\widehat{u}_{i t}-\bar{u}_{i t}\right)^{2}+2\|\bar{u}\|_{\mathrm{F}}^{2}=O_{p}\left(1+n T^{-1}\right)
$$


Using this as well as Cauchy-Schwartz inequality, we have

$$
\frac{1}{n} \sum_{i=1}^{n}\left|\widehat{u}_{i t}^{2}-u_{i t}^{2}\right| \leq\left(\frac{1}{n} \sum_{i=1}^{n}\left(\widehat{u}_{i t}-u_{i t}\right)^{2}+\frac{2}{n}\left(\sum_{i=1}^{n} u_{i t}^{2} \sum_{i=1}^{n}\left(u_{i t}-\widehat{u}_{i t}\right)^{2}\right)^{1 / 2}\right)=O_{p}\left(n^{-1 / 2}+T^{-1 / 2}\right),
$$

then by Lemmas 3(b) and 10(ii), we have

$$
\begin{aligned}
& \frac{1}{n}\left\|\sum_{i=1}^{n}\left(\widetilde{\beta}_{i}-\beta_{i}\right)\left(\widetilde{\beta}_{i}-\beta_{i}\right)^{\top}\left(\widehat{u}_{i t}^{2}-u_{i t}^{2}\right)\right\|_{\mathrm{MAX}} \leq\|\widetilde{\beta}-\beta\|_{\mathrm{MAX}}^{2} \frac{1}{n} \sum_{i=1}^{n}\left|\widehat{u}_{i t}^{2}-u_{i t}^{2}\right|=o_{p}(1), \\
& \frac{1}{n}\left\|\sum_{i=1}^{n}\left(\widetilde{\beta}_{i}-\beta_{i}\right)\left(\widetilde{\beta}_{i}-\beta_{i}\right)^{\top} u_{i t}^{2}\right\|_{\mathrm{MAX}} \leq \frac{1}{n}\|\widetilde{\beta}-\beta\|_{\mathrm{MAX}}^{2} \sum_{i=1}^{n} u_{i t}^{2}=o_{p}(1) . \\
& \frac{1}{n}\left\|\sum_{i=1}^{n} \beta_{i}\left(\widetilde{\beta}_{i}-\beta_{i}\right)^{\top} u_{i t}^{2}\right\|_{\mathrm{MAX}} \leq \frac{1}{n}\|\beta\|_{\mathrm{MAX}}\|\widetilde{\beta}-\beta\|_{\mathrm{MAX}} \sum_{i=1}^{n} u_{i t}^{2}=o_{p}(1) . \\
& \frac{1}{n}\left\|\sum_{i=1}^{n}\left(\widetilde{\beta}_{i}-\beta_{i}\right) \beta_{i}^{\top} u_{i t}^{2}\right\|_{\mathrm{MAX}} \leq \frac{1}{n}\|\beta\|_{\mathrm{MAX}}\|\widetilde{\beta}-\beta\|_{\mathrm{MAX}} \sum_{i=1}^{n} u_{i t}^{2}=o_{p}(1) . \\
& \frac{1}{n}\left\|\sum_{i=1}^{n} \beta_{i}\left(\widetilde{\beta}_{i}-\beta_{i}\right)^{\top}\left(\widehat{u}_{i t}^{2}-u_{i t}^{2}\right)\right\|_{\mathrm{MAX}} \leq\|\beta\|_{\mathrm{MAX}}\|\widetilde{\beta}-\beta\|_{\mathrm{MAX}} \frac{1}{n} \sum_{i=1}^{n}\left|\widehat{u}_{i t}^{2}-u_{i t}^{2}\right|=o_{p}(1) . \\
& \frac{1}{n}\left\|\sum_{i=1}^{n}\left(\widetilde{\beta}_{i}-\beta_{i}\right) \beta_{i}^{\top}\left(\widehat{u}_{i t}^{2}-u_{i t}^{2}\right)\right\|_{\mathrm{MAX}} \leq\|\beta\|_{\mathrm{MAX}}\|\widetilde{\beta}-\beta\|_{\mathrm{MAX}} \frac{1}{n} \sum_{i=1}^{n}\left|\widehat{u}_{i t}^{2}-u_{i t}^{2}\right|=o_{p}(1) . \\
& \frac{1}{n}\left\|\sum_{i=1}^{n} \beta_{i} \beta_{i}^{\top}\left(\widehat{u}_{i t}^{2}-u_{i t}^{2}\right)\right\|_{\mathrm{MAX}} \leq\|\beta\|_{\mathrm{MAX}}^{2} \frac{1}{n} \sum_{i=1}^{n}\left|\widehat{u}_{i t}^{2}-u_{i t}^{2}\right|=o_{p}(1) .
\end{aligned}
$$

Therefore, we have

$$
\begin{aligned}
& \frac{1}{n}\left\|\sum_{i=1}^{n}\left(H^{\top} \widehat{\beta}_{i} \widehat{\beta}_{i}^{\top} H \widehat{u}_{i t}^{2}-\beta_{i} \beta_{i}^{\top} u_{i t}^{2}\right)\right\|_{\mathrm{MAX}} \\
\leq & \frac{1}{n} \| \sum_{i=1}^{n}\left(\widetilde{\beta}_{i}-\beta_{i}\right)\left(\widetilde{\beta}_{i}-\beta_{i}\right)^{\top}\left(\widehat{u}_{i t}^{2}-u_{i t}^{2}\right)+\left(\widetilde{\beta}_{i}-\beta_{i}\right)\left(\widetilde{\beta}_{i}-\beta_{i}\right)^{\top} u_{i t}^{2}+\beta_{i}\left(\widetilde{\beta}_{i}-\beta_{i}\right)^{\top}\left(\widehat{u}_{i t}^{2}-u_{i t}^{2}\right) \\
& +\beta_{i}\left(\widetilde{\beta}_{i}-\beta_{i}\right)^{\top} u_{i t}^{2}+\left(\widetilde{\beta}_{i}-\beta_{i}\right) \beta_{i}^{\top}\left(\widehat{u}_{i t}^{2}-u_{i t}^{2}\right)+\left(\widetilde{\beta}_{i}-\beta_{i}\right) \beta_{i}^{\top} u_{i t}^{2}+\beta_{i} \beta_{i}^{\top}\left(\widehat{u}_{i t}^{2}-u_{i t}^{2}\right) \|_{\mathrm{MAX}} \\
= & o_{p}(1),
\end{aligned}
$$

which leads to the desired result.

Finally, we prove (ii) for the estimator (8). Note that by Fan et al. (2013), we have

$$
\left\|\widehat{\Sigma}^{u}-\Sigma^{u}\right\|=O_{p}\left(s_{n} \omega_{T}^{1-h}\right) .
$$

Then by (A.36) and Lemmas 3(b), 4(d), and using the fact that $\|\beta\|_{\mathrm{F}}=O_{p}\left(n^{1 / 2}\right)$ and $\left\|\Sigma^{u}\right\| \leq\left\|\Sigma^{u}\right\|_{1}=$ 
$O_{p}\left(s_{n}\right)$, we have

$$
\begin{aligned}
\frac{1}{n}\left\|(\widetilde{\beta}-\beta)^{\top}\left(\widehat{\Sigma}^{u}-\Sigma^{u}\right)(\widetilde{\beta}-\beta)\right\|_{\mathrm{MAX}} & \leq \frac{1}{n}\|\widetilde{\beta}-\beta\|_{\mathrm{F}}^{2}\left\|\widehat{\Sigma}^{u}-\Sigma^{u}\right\|=O_{p}\left(s_{n} \omega_{T}^{1-h}\left(n^{-1}+T^{-1}\right)\right), \\
\frac{1}{n}\left\|(\widetilde{\beta}-\beta)^{\top} \Sigma^{u}(\widetilde{\beta}-\beta)\right\|_{\mathrm{MAX}} & \leq \frac{1}{n}\|\widetilde{\beta}-\beta\|_{\mathrm{F}}^{2}\left\|\Sigma^{u}\right\|=O_{p}\left(s_{n}\left(n^{-1}+T^{-1}\right)\right), \\
\frac{1}{n}\left\|\beta^{\top}\left(\widehat{\Sigma}^{u}-\Sigma^{u}\right) \beta\right\|_{\mathrm{MAX}} & \leq \frac{1}{n}\|\beta\|_{\mathrm{F}}^{2}\left\|\widehat{\Sigma}^{u}-\Sigma^{u}\right\|=O_{p}\left(s_{n} \omega_{T}^{1-h}\right), \\
\frac{1}{n}\left\|\beta^{\top}\left(\widehat{\Sigma}^{u}-\Sigma^{u}\right)(\widetilde{\beta}-\beta)\right\|_{\mathrm{MAX}} & \leq \frac{1}{n}\left\|\left(\widehat{\Sigma}^{u}-\Sigma^{u}\right)(\widetilde{\beta}-\beta) \beta^{\top}\right\| \leq \frac{1}{n}\left\|\widehat{\Sigma}^{u}-\Sigma^{u}\right\|\left\|\beta^{\top}(\widetilde{\beta}-\beta)\right\| \\
& \leq \frac{K}{n}\left\|\beta^{\top}(\widetilde{\beta}-\beta)\right\|_{\mathrm{MAX}}\left\|\widehat{\Sigma}^{u}-\Sigma^{u}\right\|=O_{p}\left(s_{n} \omega_{T}^{1-h}\left(n^{-1}+T^{-1}\right)\right), \\
\frac{1}{n}\left\|\beta^{\top} \Sigma^{u}(\widetilde{\beta}-\beta)\right\|_{\mathrm{MAX}} & \leq \frac{K}{n}\left\|\beta^{\top}(\widetilde{\beta}-\beta)\right\|_{\mathrm{MAX}}\left\|\Sigma^{u}\right\|=O_{p}\left(s_{n}\left(n^{-1}+T^{-1}\right)\right) .
\end{aligned}
$$

Therefore,

$$
\begin{aligned}
& \left\|H^{\top} \widehat{\Omega} H-\Omega\right\|_{\mathrm{MAX}}=\frac{1}{n}\left\|H^{\top} \widehat{\beta}^{\top} \widehat{\Sigma}^{u} \widehat{\beta} H-\beta^{\top} \Sigma^{u} \beta\right\|_{\mathrm{MAX}} \\
\leq & \frac{1}{n}\left\|(\widetilde{\beta}-\beta)^{\top}\left(\widehat{\Sigma}^{u}-\Sigma^{u}\right)(\widetilde{\beta}-\beta)+(\widetilde{\beta}-\beta)^{\top} \Sigma^{u}(\widetilde{\beta}-\beta)\right\|_{\mathrm{MAX}}+\frac{1}{n}\left\|\beta^{\top}\left(\widehat{\Sigma}^{u}-\Sigma^{u}\right) \beta\right\|_{\mathrm{MAX}} \\
& +\frac{1}{n}\left\|\beta^{\top}\left(\widehat{\Sigma}^{u}-\Sigma^{u}\right)(\widetilde{\beta}-\beta)+(\widetilde{\beta}-\beta)^{\top}\left(\widehat{\Sigma}^{u}-\Sigma^{u}\right) \beta\right\|_{\mathrm{MAX}}+\frac{1}{n}\left\|\beta^{\top} \Sigma^{u}(\widetilde{\beta}-\beta)+(\widetilde{\beta}-\beta)^{\top} \Sigma^{u} \beta\right\|_{\mathrm{MAX}} \\
= & O_{p}\left(s_{n}\left(\omega_{T}^{1-h}+n^{-1}+T^{-1}\right)\right)=o_{p}(1),
\end{aligned}
$$

which concludes the proof.

Proof of Theorem 8. By the conditioning argument, we assume $\widehat{p}=p$. By (A.19), we have

$$
\widehat{\eta}-\eta H^{-1}=T^{-1} \bar{Z} \bar{V}^{\top} H^{\top}+O_{p}\left(n^{-1}+T^{-1}\right)
$$

Therefore, when $n^{-2} T=o(1)$, we can rewrite:

$$
\widehat{W}=T\left(\eta H^{-1}+T^{-1} \bar{Z} \bar{V}^{\top} H^{\top}\right)\left(\widehat{\Sigma}_{v}^{-1} \widehat{\Pi}_{11} \widehat{\Sigma}_{v}^{-1}\right)^{-1}\left(\eta H^{-1}+T^{-1} \bar{Z} \bar{V}^{\top} H^{\top}\right)^{\top}+o_{p}(1) .
$$

Under $\mathbb{H}_{0}: \eta=0$, we have

$$
\widehat{W}=\left(T^{-1 / 2} \bar{Z} \bar{V}^{\top}\right)\left(H^{-1} \widehat{\Sigma}_{v}^{-1} \widehat{\Pi}_{11} \widehat{\Sigma}_{v}^{-1} H^{-\top}\right)^{-1}\left(T^{-1 / 2} \bar{Z} \bar{V}^{\top}\right)^{\top}+o_{p}(1) .
$$

By Assumption 11, to show $\widehat{W} \stackrel{\mathcal{L}}{\longrightarrow} \chi_{p}^{2}$ under $\mathbb{H}_{0}$, it is sufficient to establish that

$$
H^{-1} \widehat{\Sigma}_{v}^{-1} \widehat{\Pi}_{11} \widehat{\Sigma}_{v}^{-1} H^{-\top} \stackrel{p}{\longrightarrow} \Pi_{11} .
$$

By the same argument as in the proof of (A.32) and the fact that $\widehat{\Sigma}_{v}=\mathbb{I}_{d}$, we have

$$
H^{-1} \widehat{\Pi}_{11} H^{-\top} \stackrel{p}{\longrightarrow} \Pi_{11}
$$


which leads to the first claim. The second claim is straightforward because $H$ is invertible with probability approaching $1,\|H\|=O_{p}(1)$ by Lemma 2 , and $T^{-1} \bar{Z} \bar{V}^{\top}=O_{p}\left(T^{-1 / 2}\right)$ by Assumption 6 . 